\title{
Characterization of Optimal Carbon Nanotubes Under Stretching and Validation of the Cauchy-Born Rule
}

\author{
Manuel Friedrich, Edoardo Mainini, Paolo Piovano \& \\ Ulisse Stefanelli
}

Communicated by A. BRAIDES

\begin{abstract}
Carbon nanotubes are modeled as point configurations and investigated by minimizing configurational energies including two- and three-body interactions. Optimal configurations are identified with local minima and their fine geometry is fully characterized in terms of lower-dimensional problems. Under moderate tension, we prove the existence of periodic local minimizers, which indeed validates the so-called Cauchy-Born rule in this setting.
\end{abstract}

\section{Contents}

1. Introduction . . . . . . . . . . . . . . . . . . . . . 466

2. Carbon-Nanotube Geometry . . . . . . . . . . . . . . . . . . . . . . . . . . . 469

2.1. Configurational Energy . . . . . . . . . . . . . . . . . . . . 469

2.2. Geometry of Zigzag Nanotubes . . . . . . . . . . . . . . . . . . . . 470

3. Main Results . . . . . . . . . . . . . . . . . . . . . . . . . . . . . . . . . . 474

3.1. Unstrechted Nanotubes . . . . . . . . . . . . . . . . . . . . . . . . 474

3.2. Nanotubes Under Stretching ． . . . . . . . . . . . . . . . . . 475

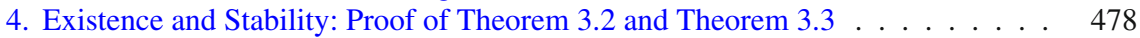

5. Symmetry Defect Controls Angle Defect: Proof of Lemma 4.1 . . . . . . . . . . 486

6. Properties of the Reduced Energy: Proof of Lemma 4.2, Proposition 4.3, and Proposition $3.4 \ldots \ldots \ldots \ldots \ldots$

6.1. Proof of Lemma $4.2 \ldots \ldots$. . . . . . . . . . . . . . . . . 488

6.2. Convexity of the Reduced Energy . . . . . . . . . . . . . . . . . . . . . 488

6.3. Proof of Proposition 4.3 and Proposition $3.4 \ldots \ldots$. . . . . . . . . . . . . . 495

7. Energy Defect Controls Symmetry Defect: Proof of Theorem 4.4 . . . . . . . . 497

7.1. Relation Between Atomic Positions, Bonds, and Angles . . . . . . . . . . . 497

7.2. Convexity of the Cell Energy . . . . . . . . . . . . . . . . . . . . 503

7.3. Proof of Theorem $4.4 \ldots \ldots \ldots$

References . . . . . . . . . . . . . . . . . . . . . . 514 


\section{Introduction}

Nanostructured carbon has emerged over the last two decades as one of the most promising materials available to mankind. The discovery of fullerenes [48,49], followed by that of carbon nanotubes [41] and graphene [37,62], sparked an interest in low-dimensional materials. The fascinating electronic and mechanical properties of single-atom-thick surfaces and structures are believed to offer unprecedented opportunities for innovative applications, ranging from next-generation electronics to pharmacology, to batteries and solar cells $[39,58,59]$. New findings are emerging at an always increasing pace, cutting across materials science, physics, and chemistry, and extending from fundamental science to novel applications [23,61].

Carbon nanotubes are long, hollow structures exhibiting cylindrical symmetry [18]. Their walls consist of a single (or multiple) one-atom-thick layer of carbon atoms forming $s p^{2}$ covalent bonds [12] arranged in a hexagonal pattern. This molecular structure is responsible for amazing mechanical properties: carbon nanotubes are presently among the strongest and stiffest known materials with a nominal Young's modulus [47,71] of $1 \mathrm{TPa}$ and ideal strength greater than $100 \mathrm{MPa}$ [3]. In addition, they are electrically and thermally conductive, chemically sensitive, transparent, and light weight [72]. Nanotubes can be visualized as the result of rolling up a patch of a regular hexagonal lattice. Depending on the different possible realizations of this rolling-up, different topologies may arise, giving rise to zigzag, armchair, and chiral nanotubes. These topologies are believed to have a specific impact on the mechanical and electronic properties of the nanotube, which can range from highly conducting to semiconducting $[9,10]$.

In contrast to the ever-growing material knowledge, the rigorous mathematical description of two-dimensional carbon systems is considerably less developed. $A b$ initio atomistic models are believed to accurately describe some features of the carbon nanotube geometry and mechanics $[54,65,76]$. These methods are nevertheless computational in nature and cannot handle a very large number of atoms due to the rapid increase in computational complexity. On the other hand, a number of continuum mechanics approaches have been proposed where carbon nanotubes are modeled as rods [63], shells [3,4,28,66], or solids [73]. These bring the advantage of possibly dealing with long structures, at the price however of a less accurate description of the detailed microscopic behavior.

The unique mechanical behavior of nanotubes under stretching is a crucial feature of these structures. As such, it has attracted attention from the theoretical $[4,29,66,79]$, the computational $[1,9,40,44]$, and the experimental side $[17,47,74,77]$. Still, a reliable description of nanotubes under stretching requires one to correctly resolve the atomic scale and, simultaneously, to rigorously deal with the whole structure. We hence resort to the classical frame of molecular mechanics $[2,53,64]$ which identifies carbon nanotubes with point configurations $\left\{x_{1}, \ldots, x_{n}\right\} \in \mathbb{R}^{3 n}$ corresponding to their atomic positions. The atoms are interacting via a configurational energy $E=E\left(x_{1}, \ldots, x_{n}\right)$ given in terms of classical potentials and taking into account both attractive-repulsive two-body interactions, minimized at a certain bond length, and three-body terms favoring specific angles between bonds $[6,69,70]$. The $s p^{2}$-type covalent bonding implies that each atom 
has exactly three first neighbors and that bond angles of $2 \pi / 3$ are energetically preferred [12]. The reader is referred to [16,20,32,57,68] for a collection of results on local and global minimizers in this setting and to [27,51] for additional results on carbon structures.

The focus of this paper is to show the local minimality of periodic configurations, both in the unstreched case and under the effect of small stretching. More specifically, we prove that, by applying a small stretching to a zigzag nanotube, the energy $E$ is locally strictly minimized by a specific periodic configuration where all atoms see the same local configuration (Theorem 3.3). Local minimality is here checked with respect to all small perturbations in $\mathbb{R}^{3 n}$, namely not restricting a priori to periodic perturbations. On the contrary, periodicity is proved here to emerge as effect of the global variational nature of the problem.

The novelty of this result is threefold. At first, given the periodicity of the mentioned local minimizers, the actual configuration in $\mathbb{R}^{3 n}$ can be determined by solving a simple minimization problem in $\mathbb{R}^{2}$, which consists in identifying the length of two specific bond lengths between neighboring atoms. This is indeed the standpoint of a number of contributions, see [1,8,30,31,43,44,46,50] among many others, where nevertheless periodicity is a priori assumed. In this regard, our result offers a justification for these lower-dimensional approaches. Our assumptions on $E$ are kept fairly general in order to include the menagerie of different possible choices for energy terms which have been implemented in computational chemistry codes $[7,11,38,60,75]$. A by-product of our results is hence the cross-validation of these choices in view of their capability of describing carbon nanotube geometries.

Secondly, we rigorously check that, also in the presence of small stretching, the geometrical model obtained via local minimization corresponds neither to the classical rolled-up model $[18,19,45]$, where two out of three bond angles at each atom are $2 \pi / 3$, nor to the polyhedral model $[14,15,52]$, where all bond angles are equal. The optimal configuration lies between these two (Proposition 3.4), a fact which remarkably corresponds to measurements on very thin carbon nanotubes [80]. Moreover, in accordance with the results in [44], local minimizers are generically characterized by two different bond lengths.

Finally, our result proves the validity of the so-called Cauchy-Born rule for carbon nanotubes: by imposing a small tension, the periodicity cell deforms correspondingly and global periodicity is preserved. This fact rests at the basis of a possible elastic theory for carbon nanotubes. As a matter of fact, such periodicity is invariably assumed in a number of different contributions, see [4,29,40,79] among others, and then exploited in order to compute tensile strength as well as stretched geometries. Here again our results provide a theoretical justification of such approaches.

While the Cauchy-Born rule plays a pivotal role in mechanics [25,26,78], rigorous results are scarce. Among these we mention $[13,36]$, which assess its validity within two- and $d$-dimensional cubic mass-spring systems, respectively. More general interactions are considered in [21,22], where the Cauchy-Born rule is investigated under a specific ellipticity condition applying to the triangular and hexagonal lattice, both in the static and the dynamic case. Our result is, to the best of our knowledge, the first one dealing with a three-dimensional structure which is 
not a subset of a Bravais lattice nor of a multilattice. Note though the Saint Venant principle in [24], which corresponds to the validity of an approximate version of the Cauchy-Born rule, up to a small error. However, the setting of [24] is quite different from the present one, where long-range purely two-body interactions are considered.

This work is the culmination of a series on the geometry and mechanics of nanotubes $[55,56]$. The theoretical outcomes of this paper have been predicted computationally in [55], where stability of periodic configurations have been investigated with Monte Carlo techniques, both for zigzag and armchair topologies under moderate displacements. A first step toward a rigorous analytical result has been obtained in [56] for both zigzag and armchair topologies under no stretching. In [56], stability is checked against a number of non-periodic perturbations fulfilling a specific structural constraint, which is related to the nonplanarity of the hexagonal cells induced by the local geometry of the nanotube. Here, we remove such constraints and consider all small perturbations, even in presence of stretching.

Indeed, removing the structural assumption and extending the result of [56] to the present fully general setting requires a remarkably deeper analysis. In a nutshell, one has to reduce to a cell problem and solve it. The actual realization of this program poses however substantial technical challenges and relies on a combination of perturbative and convexity techniques.

Whereas the proof in [56] was essentially based on the convexity of the energy given by the three bond angles at one atom, in the present context we have to reduce to a cell which includes eight atoms and is slightly nonplanar. The convexity of cell energies for various Bravais lattices has already been investigated in the literature $[13,34,36,67]$, particularly for problems related to the validation of the CauchyBorn rule. In our setting, however, we need to deal with an almost planar structure embedded in the three-dimensional space and therefore, to confirm convexity of the cell energy, a careful analysis in terms of the nonplanarity is necessary, see Section 7.2 and Theorem 7.6. In this context, an additional difficulty lies in the fact that the reference configuration of the cell is not a stress-free state.

The convexity is then crucially exploited in order to obtain a quantitative control of the energy defect in terms of the symmetry defect produced by symmetrizing a cell (Theorem 4.4). On the other hand, a second quantitative estimate provides a bound on the defect in the nonplanarity of the cell (called angle defect) with respect to the symmetry defect of the cell (Lemma 4.1). The detailed combination of these two estimates and a convexity and monotonicity argument (Proposition 4.3) proves that ground states necessarily have symmetric cells, from which our stability result follows (Theorem 3.3).

The validation of the Cauchy Born rule essentially relies on the application of a slicing technique which has also been used in [34] in a more general setting: one reduces the problem to a chain of cells along the diameter of the structure and shows that identical deformation of each cell is energetically favorable. In the present context, however, additional slicing arguments along the cross sections of the nanotube are necessary in order to identify correctly the nonplanarity of each hexagonal cell. 
The paper is organized as follows. In Section 2 we introduce some notation and the mathematical setting. Section 3 collects our main results. In Section 4 we present the proof strategy, the essential auxiliary statements (Lemma 4.1, Theorem 4.4), and the proof of Theorem 3.3. The proofs of the various necessary ingredients are postponed to Sections 5-7.

\section{Carbon-Nanotube Geometry}

The aim of this section is to introduce some notation and the nanotube configurational energy. Let us start by introducing the mathematical setting as well as some preliminary observations.

As mentioned above, carbon nanotubes (nanotubes, in the following) are modeled by configurations of atoms, i.e., collections of points in $\mathbb{R}^{3}$ representing the atomic sites. Nanotubes are very long structures, measuring up to $10^{7}$ times their diameter. As such, we shall not be concerned with describing the fine nanotube geometry close to their ends. We thus restrict our attention to periodic configurations, i.e., configurations that are invariant with respect to a translation of a certain period in the direction of the nanotube axis. Without loss of generality we consider only nanotubes with axis in the $e_{1}:=(1,0,0)$ direction. Therefore, a nanotube is identified with a configuration

$$
\mathcal{C}:=C_{n}+L e_{1} \mathbb{Z}
$$

where $L>0$ is the period of $\mathcal{C}$ and $C_{n}:=\left\{x_{1}, \ldots, x_{n}\right\}$ is a collection of $n$ points $x_{i} \in \mathbb{R}^{3}$ such that $x_{i} \cdot e_{1} \in[0, L)$. In the following, we will refer to $C_{n}$ as the $n$-cell of $\mathcal{C}$, and since $\mathcal{C}$ is characterized by its $n$-cell $C_{n}$ and its period $L$, we will systematically identify the periodic configuration $\mathcal{C}$ with the couple $\left(C_{n}, L\right)$, i.e., $\mathcal{C}=\left(C_{n}, L\right)$.

\subsection{Configurational Energy}

We now introduce the configurational energy $E$ of a nanotube $\mathcal{C}$, and we detail the hypotheses on $E$ that we assume throughout the paper. We aim here at minimal assumptions in order to include in the analysis most of the many different possible choices for energy terms that have been successfully implemented in computational chemistry codes $[7,11,38,60,75]$.

The energy $E$ is given by the sum of two contributions, respectively accounting for two-body and three-body interactions among particles that are respectively modelled by the potentials $v_{2}$ and $v_{3}$, see (1).

We assume that the two-body potential $v_{2}:(0, \infty) \rightarrow[-1, \infty)$ is smooth and attains its minimum value only at 1 with $v_{2}(1)=-1$ and $v_{2}^{\prime \prime}(1)>0$. Moreover, we ask $v_{2}$ to be short-ranged, that is to vanish shortly after 1 . For the sake of definiteness, let us define $v_{2}(r)=0$ for $r \geqq 1.1$. These assumptions reflect the nature of covalent atomic bonding in carbon favoring a specific interatomic distance, here normalized to 1 . 
We say that two particles $x, y \in \mathcal{C}$ are bonded if $|x-y|<1.1$, and we refer to the graph formed by all the bonds as the bond graph of $\mathcal{C}$. Taking into account periodicity, this amounts to considering two particles $x_{i}$ and $x_{j}$ of the $n$-cell $C_{n}$ of $\mathcal{C}$ to be bonded if $\left|x_{i}-x_{j}\right|_{L}<1$.1, where $|\cdot|_{L}$ is the distance modulo $L$ defined by

$$
\left|x_{i}-x_{j}\right|_{L}:=\min _{t \in\{-1,0,+1\}}\left|x_{i}-x_{j}+L t e_{1}\right|
$$

for every $x_{i}, x_{j} \in C_{n}$. Let us denote by $\mathcal{N}$ the set of all couples of indices corresponding to bonded particles, i.e.,

$$
\mathcal{N}:=\left\{(i, j): x_{i}, x_{j} \in C_{n}, i \neq j, \text { and }\left|x_{i}-x_{j}\right|_{L}<1.1\right\} .
$$

The three-body potential $v_{3}:[0,2 \pi] \rightarrow[0, \infty)$ is assumed to be smooth and symmetric around $\pi$, namely $v_{3}(\alpha)=v_{3}(2 \pi-\alpha)$. Moreover, we suppose that the minimum value 0 is attained only at $2 \pi / 3$ and $4 \pi / 3$ with $v_{3}^{\prime \prime}(2 \pi / 3)>0$. Let $\mathcal{T}$ be the index set of the triples corresponding to first-neighboring particles, i.e.,

$$
\mathcal{T}:=\{(i, j, k): i \neq k,(i, j) \in \mathcal{N} \text { and }(j, k) \in \mathcal{N}\} .
$$

For all triples $(i, j, k) \in \mathcal{T}$ we denote by $\alpha_{i j k} \in[0, \pi]$ the bond angle formed by the vectors $x_{i}-x_{j}$ and $x_{k}-x_{j}$. The assumptions on $v_{3}$ reflect the basic geometry of carbon bonding in a nanotube: Each atom presents three $s p^{2}$-hybridized orbitals, which tend to form $2 \pi / 3$ angles.

The configurational energy $E$ of a nanotube $\mathcal{C}=\left(C_{n}, L\right)$ is now defined by

$$
E(\mathcal{C})=E\left(C_{n}, L\right):=\frac{1}{2} \sum_{(i, j) \in \mathcal{N}} v_{2}\left(\left|x_{i}-x_{j}\right|_{L}\right)+\frac{1}{2} \sum_{(i, j, k) \in \mathcal{T}} v_{3}\left(\alpha_{i j k}\right),
$$

where the factors $1 / 2$ are included to avoid double-counting the interactions among same atoms. Let us mention that the smoothness assumptions on $v_{2}$ and $v_{3}$ are for the sake of maximizing simplicity rather than generality and could be weakened. Observe that our assumptions are generally satisfied by classical interaction potentials for carbon (see $[69,70]$ ). Since the energy $E$ is clearly rotationally and translationally invariant, in the following we will tacitly assume that all statements are to be considered up to isometries. We say that a nanotube $\mathcal{C}=\left(C_{n}, L\right)$ is stable if $\left(C_{n}, L\right)$ is a strict local minimizer of the interaction energy $E$.

\subsection{Geometry of Zigzag Nanotubes}

We now introduce a specific two-parameter family of nanotubes which will play a crucial role in the following. This is the family of so-called zigzag nanotubes having the minimal period $\mu>0$. The term zigzag refers to a specific topology of nanotubes, which can be visualized as the result of a rolling-up of a graphene sheet along a specific lattice direction, see Fig. 1.

The resulting three-dimensional structure is depicted in Fig. 2. Note that our preference for the zigzag topology is solely motivated by the sake of definiteness. The other classical choice, namely the so-called armchair topology, could be 


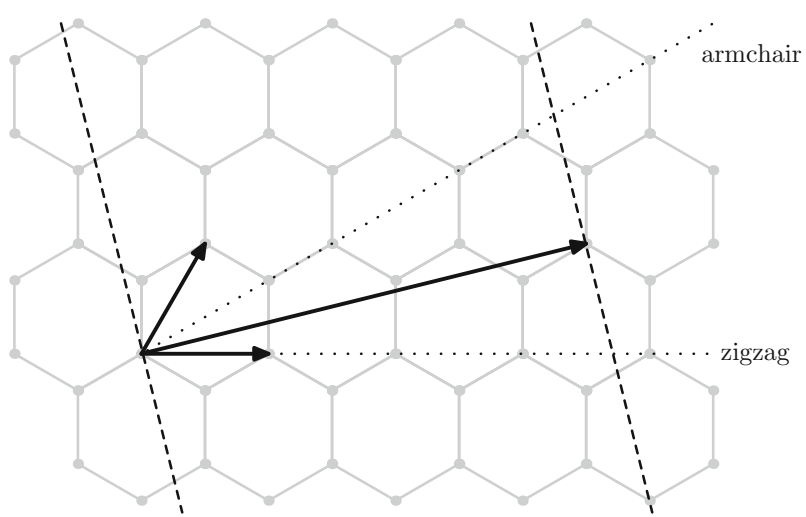

Fig. 1. Rolling-up a graphene sheet to a zigzag nanotube: the vector illustrates the identification of the two dashed vertical lines. The term zigzag refers to the orientation of this vector with respect to bonds. Different vectors correspond indeed to different nanotube topologies. The dotted line indicates the identification direction for armchair nanotubes

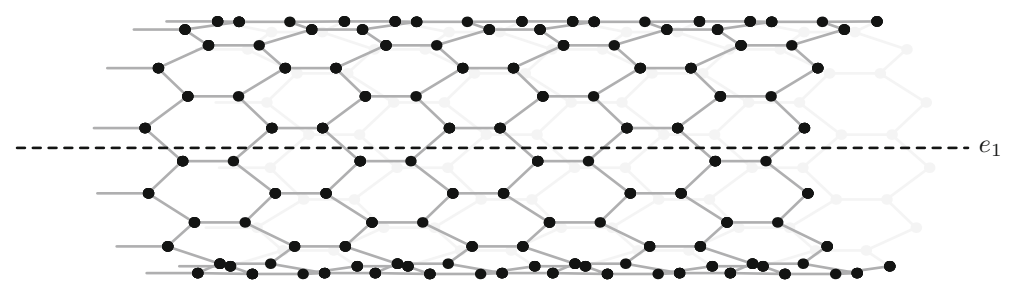

Fig. 2. Zigzag nanotube

considered as well. The reader is referred to [56] for some results on unstretched armchair geometries.

We let $\ell \in \mathbb{N}, \ell>3$, and define the family $\mathscr{F}(\mu)$ as the collection of all configurations that, up to isometries, coincide with

$$
\begin{gathered}
\left\{\left(k\left(\lambda_{1}+\sigma\right)+j\left(2 \sigma+2 \lambda_{1}\right)+l\left(2 \sigma+\lambda_{1}\right), \rho \cos \left(\frac{\pi(2 i+k)}{\ell}\right),\right.\right. \\
\left.\left.\rho \sin \left(\frac{\pi(2 i+k)}{\ell}\right)\right) \mid i=1, \ldots, \ell, j \in \mathbb{Z}, k, l \in\{0,1\}\right\}
\end{gathered}
$$

for some choice of

$\lambda_{1} \in(0, \mu / 2), \quad \lambda_{2} \in(0, \mu / 2), \quad \sigma \in(0, \mu / 2), \quad$ and $\quad \rho \in\left(0, \frac{\mu}{4 \sin (\pi /(2 \ell))}\right)$

such that

$$
2 \sigma+2 \lambda_{1}=\mu, \quad \sigma^{2}+4 \rho^{2} \sin ^{2}\left(\frac{\pi}{2 \ell}\right)=\lambda_{2}^{2} .
$$

Of course, the configurations in $\mathscr{F}(\mu)$ are periodic with minimal period $\mu$. The parameter $\rho$ indicates the diameter of the tube and $\lambda_{1}, \lambda_{2}$ are the two possibly 
different lengths of the covalent bonds in each hexagon of the tube, where the bonds of length $\lambda_{1}$ are oriented in the $e_{1}$ direction (see Fig. 4).

These configurations are objective [42]; they are obtained as orbits of two points under the action of a prescribed isometry group. The latter group is generated by a translation and by a translation combined with a rotation about the $e_{1}$-axis. Notice that our definition slightly differs from the one adopted in $[55,56]$ in the sense that for fixed $i, k$ the points identified by the quadruples $(i, j, k, l)$ for $j \in \mathbb{Z}, l \in\{0,1\}$ lie on a line parallel to $e_{1}$ (see Fig. 3).

For fixed $\mu>0, \mathscr{F}(\mu)$ is a two-parameter smooth family of configurations since each configuration in $\mathscr{F}(\mu)$ is uniquely determined by $\lambda_{1}$ and $\lambda_{2}$ by taking relation (3) into account. Later we will consider different values for the minimal period $\mu$ in order to model nanotubes under stretching.

We state the following basic geometric properties of configurations in $\mathscr{F}(\mu)$; see Fig. 3 (the analogous properties in the case $\lambda_{1}=\lambda_{2}=1$ have already been discussed in [55]):

Proposition 2.1. (Geometric structure of zigzag nanotubes). Let $\mathcal{F} \in \mathscr{F}(\mu)$. Then:

(a) Atoms in $\mathcal{F}$ lie on the surface of a cylinder with radius $\rho$ and axis $e_{1}$.

(b) Atoms in $\mathcal{F}$ are arranged in planar sections, perpendicular to $e_{1}$, obtained by fixing $j, k$, and $l$ in (2). Each of the sections contains exactly $\ell$ atoms, arranged at the vertices of a regular $\ell$-gon. For each section, the two closest sections are at distance $\sigma$ and $\lambda_{1}$, respectively.

(c) The configuration $\mathcal{F}$ is invariant under a rotation of $2 \pi / \ell$ around $e_{1}$, under the translation $\mu e_{1}$, and under a transformation consisting of a rotation of $\pi / \ell$ around $e_{1}$ and a translation along the vector $\left(\lambda_{1}+\sigma\right) e_{1}$ (see Fig. 4).

(d) Let $i \in\{1, \ldots, \ell\}, j \in \mathbb{Z}$ and $k, l \in\{0,1\}$ : the quadruple $(i, j, k, l)$ identifies points of $\mathcal{F}$, denoted by $x_{i, k}^{j, l}$, where $(0, j, k, l)$ is identified with $(\ell, j, k, l)$. Given $x_{i, 0}^{j, 0} \in \mathcal{F}$, the two points $x_{i, 1}^{j-1,1}, x_{i-1,1}^{j-1,1}$ have distance $\lambda_{2}$ and $x_{i, 0}^{j-1,1}$ has distance $\lambda_{1}$ from $x_{i, 0}^{j, 0}$. For $x_{i, 0}^{j, 1}$, the distance of $x_{i, 1}^{j, 0}$ and $x_{i-1,1}^{j, 0}$ is $\lambda_{2}$ and the distance from $x_{i, 0}^{j+1,0}$ is $\lambda_{1}$. See Fig. 3 for the analogous notation of $x_{i, 1}^{j, 0}$ and $x_{i, 1}^{j, 1}$.

Notice that for fixed $\lambda_{1}$ and $\lambda_{2}$ the other parameters range between two degenerate cases: $\rho=0$ (the cylinder is reduced to its axis) and $\sigma=0$ (sections collide). We shall however impose further restrictions, for each atom should have three bonds. In particular, the only three bonds per atom should be the ones identified by point (d) of Proposition 2.1. By recalling that two particles are bonded if their distance is less than the reference value 1.1, since the distance between two consecutive sections is either $\lambda_{1}$ or $\sigma$, we require $\lambda_{1}>0.9$ and $\sigma>0.2$. Additionally, we require $\lambda_{1}, \lambda_{2}<1$.1, which also implies $\sigma<1.1$ by (3). On the other hand, on each section, the edge of the regular $\ell$-gon should be greater than 1.1. Such length is given by $2 \rho \sin \gamma_{\ell}$, where $\gamma_{\ell}$ is the internal angle of a regular $2 \ell$-gon, i.e.,

$$
\gamma_{\ell}:=\pi\left(1-\frac{1}{\ell}\right) .
$$




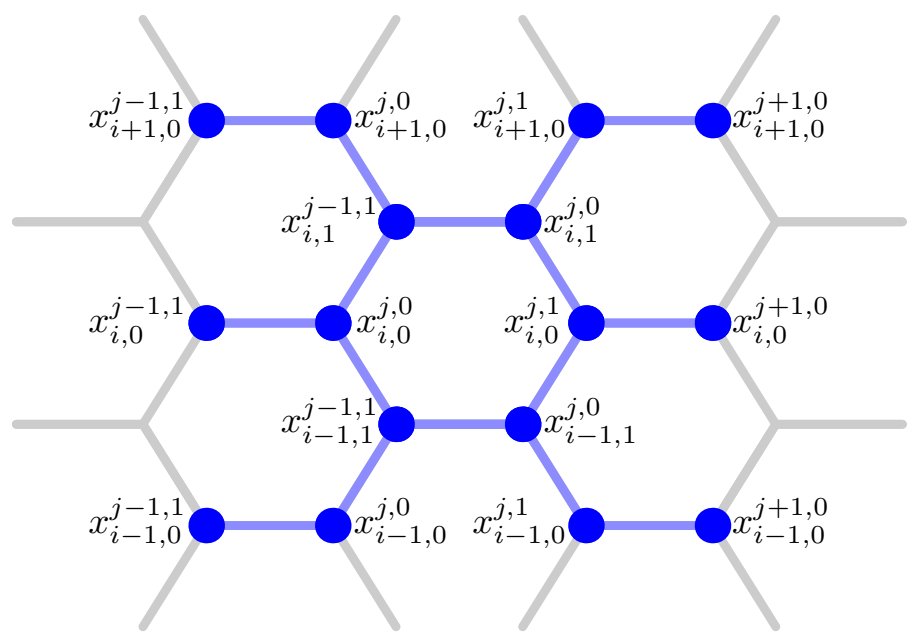

Fig. 3. Configuration points are identified by quadruples $(i, j, k, l)$ for $i=1, \ldots, \ell, j \in \mathbb{Z}$, and $k, l \in\{0,1\}$

Therefore, we need to impose $\rho>\rho^{-}:=0.55 / \sin \gamma_{\ell}$. With these restrictions we have the following:

Proposition 2.2. (Parametrization of the family). Let $\mathcal{F} \in \mathscr{F}(\mu)$ with $\rho>\rho^{-}$, $\sigma>0.2$ and $\lambda_{1}, \lambda_{2} \in(0.9,1.1)$. Then, all atoms in $\mathcal{F}$ have exactly three (firstnearest) neighbors, two at distance $\lambda_{2}$ and one at distance $\lambda_{1}$, where the bond corresponding to the latter neighbor is parallel to $e_{1}$. Among the corresponding three bond angles, which are smaller than $\pi$, two have amplitude $\alpha$ (the ones involving atoms in three different sections), and the third has amplitude $\beta$ (see Fig. 4), where $\alpha \in(\pi / 2, \pi)$ is obtained from

$$
\sin \alpha=\sqrt{1-\left(\sigma / \lambda_{2}\right)^{2}}=2\left(\rho / \lambda_{2}\right) \sin \left(\frac{\pi}{2 \ell}\right)
$$

and $\beta \in(\pi / 2, \pi)$ is given by

$$
\beta=\beta\left(\alpha, \gamma_{\ell}\right):=2 \arcsin \left(\sin \alpha \sin \frac{\gamma_{\ell}}{2}\right)
$$

The proof for the case $\lambda_{1}=\lambda_{2}=1$ was detailed in [55]. The extension to our setting is a straightforward adaption and is therefore omitted. As already mentioned, the collection $\mathscr{F}(\mu)$ is a two-parameter family where all its configurations are uniquely determined by the specification of $\lambda_{1}$ and $\lambda_{2}$. The corresponding element will be denoted by $\mathcal{F}_{\lambda_{1}, \lambda_{2}, \mu}$. Restricting the minimal period $\mu$ to the interval $(2.6,3.1)$ we observe by (3) and an elementary computation that the constraints $\lambda_{1}, \lambda_{2} \in(0.9,1.1)$ and $\ell>3$ automatically imply $0.2<\sigma<0.65$ and $\rho>\rho^{-}$. Therefore, the assumptions of Proposition 2.2 hold. 


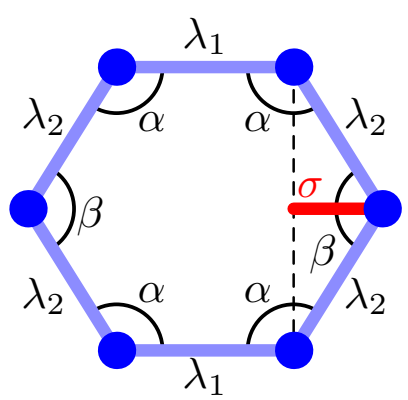

Fig. 4. The bond lengths and the angles for the hexagon of a configuration in $\mathscr{F}(\mu)$ are represented. A segment representing $\sigma$ is drawn in red

\section{Main Results}

In this section we collect our main results. The corresponding proofs will then be presented in Sections 4-7.

For a fixed integer $\ell>3$, let us consider a configuration $\mathcal{F}$ in the family $\mathscr{F}(\mu)$. As $\mathcal{F}$ is periodic, it can be identified with the couple $\left(F_{n}, L\right)$, where $F_{n}$ is the corresponding $n$-cell $(n=4 m \ell$ for some $m \in \mathbb{N})$, and

$$
L=L_{m}^{\mu}:=m \mu
$$

is the period parameter, corresponding to the cell length (notice that for $m=1$ we get the minimal period of the configuration). In view of (1) and the properties stated in Proposition 2.2, the energy can be written as

$$
E(\mathcal{F})=E\left(F_{n}, L_{m}^{\mu}\right)=\frac{n}{2}\left(v_{2}\left(\lambda_{1}\right)+2 v_{2}\left(\lambda_{2}\right)\right)+n\left(2 v_{3}(\alpha)+v_{3}\left(\beta\left(\alpha, \gamma_{\ell}\right)\right)\right) .
$$

\subsection{Unstrechted Nanotubes}

A first natural problem to be considered is the energy minimization restricted to the families $\mathscr{F}(\mu)$, with the values of $\mu$ in the reference interval $\mu \in(2.6,3.1)$. Let us denote by $\mathcal{F}_{\lambda_{1}, \lambda_{2}, \mu}$ an element of $\mathscr{F}(\mu)$ with bond lengths $\lambda_{1}, \lambda_{2}$. If we minimize among nanotubes $\mathcal{F}_{\lambda_{1}, \lambda_{2}, \mu}$ with respect to $\mu \in(2.6,3.1)$ and $\lambda_{1}, \lambda_{2}$ in a neighborhood of 1 , we reduce to the case $\lambda_{1}=\lambda_{2}=1$. Indeed, we can replace $\lambda_{1}, \lambda_{2}$ by 1 , leave $\alpha$ unchanged, and choose $\mu$ according to (3) and (5) such that the energy (8) decreases.

We notice that $\left\{\mathcal{F}_{1,1, \mu} \mid \mu \in(2.6,3.1)\right\}$ is a one-parameter family. It follows from Proposition 2.2 and (3) that this family can also be parametrized in terms of the bond angle $\alpha$ introduced in Proposition 2.2 using the relation $\mu=2(1-\cos \alpha)$. We indicate these configurations by $\mathcal{G}_{\alpha}$.

As already discussed in [55], there are two specific angles $\alpha_{\ell}^{\text {ch }}<\alpha^{\text {ru }}$ corresponding to the rolled-up $[18,19]$ and polyhedral $[14,15]$ configuration, respectively, with $\alpha^{\mathrm{ru}}=2 \pi / 3$ and $\alpha_{\ell}^{\mathrm{ch}}$ being the unique solution of the equation $\beta\left(\alpha_{\ell}^{\mathrm{ch}}, \gamma_{\ell}\right)=\alpha_{\ell}^{\mathrm{ch}}$ in $(\arccos (-0.4), \arccos (-0.6))$. The one variable minimization problem for the map $\alpha \mapsto E\left(\mathcal{G}_{\alpha}\right)$ has been investigated in [55, Theorem 4.3]. 
Proposition 3.1. (Existence and uniqueness of minimizer: Unstretched case). There exist an open interval $A$ and $\ell_{0} \in \mathbb{N}$ only depending on $v_{3}$ such that the following holds for all $\ell \geqq \ell_{0}$. There is a unique angle $\alpha_{\ell}^{\text {us }} \in A$ such that $\mathcal{G}_{\alpha_{\ell}}$ minimizes the energy $E$ in the class $\left\{\mathcal{G}_{\alpha} \mid \alpha \in A\right\}$. Moreover, one has $\alpha_{\ell}^{\mathrm{us}} \in\left(\alpha_{\ell}^{\mathrm{ch}}, \alpha^{\mathrm{ru}}\right) \subset A$.

Let us report the idea of the proof. Exploiting the monotonicity properties of $v_{3}$ and $\beta$ (the latter being decreasing as a function of $\alpha$ ), one derives that the minimum is attained for $\alpha$ in a small left neighborhood $I$ of $2 \pi / 3$, e.g., $I:=(2 \pi / 3-\sigma, 2 \pi / 3]$ for some small $\sigma>0$. Using in addition the convexity of $v_{3}$ and the concavity of $\beta$, it follows that $\alpha \mapsto E(\mathcal{F})=-3 n / 2+n\left(2 v_{3}(\alpha)+v_{3}\left(\beta\left(\alpha, \gamma_{\ell}\right)\right)\right)$ is strictly convex in $I$, which implies the assertion.

The result in particular shows that neither the polyhedral nor the rolled-up configuration is a local minimizer of the energy $E$. The corresponding minimal period of the nanotube is given by

$$
\mu_{\ell}^{\mathrm{us}}:=2-2 \cos \alpha_{\ell}^{\mathrm{us}} \text {, }
$$

cf. (3) and (5), and we notice $\mathcal{G}_{\alpha_{\ell}^{\text {us }}}=\mathcal{F}_{1,1, \mu_{\ell}^{\text {us }}}$. Nanotubes with $\mu=\mu_{\ell}^{\text {us }}$ will be referred to as unstretched nanotubes.

The aim of $[55,56]$ was to prove that $\mathcal{G}_{\alpha_{\ell}}$ us is a local minimizer. This has been illustrated numerically in [55] and checked analytically in [56], for a restricted class of perturbations. Our stability result Theorem 3.3 below delivers an analytical proof of stability with respect to all small perturbations. As such, it generalizes and improves known results, even in the unstreched case.

\subsection{Nanotubes Under Stretching}

Let us now move forward to the case of stretched nanotubes. This corresponds to choosing $\mu \neq \mu_{\ell}^{\text {us }}$. Indeed, we impose a tensile or compressive stress on the nanotube by simply modifying its minimal period. Given the role of periodicity in the definition of the energy $E$, see (1), this has the net effect of stretching/compressing the structure. Note that this action on the structure is very general. In particular, it includes, without reducing to, imposed Dirichlet boundary conditions, where only the first coordinate of the boundary atoms is prescribed. For fixed $\mu \in(2.6,3.1)$ we consider the minimization problem

$$
E_{\min }(\mu)=\min \left\{E\left(\mathcal{F}_{\lambda_{1}, \lambda_{2}, \mu}\right) \mid \mathcal{F}_{\lambda_{1}, \lambda_{2}, \mu} \in \mathscr{F}(\mu), \lambda_{1}, \lambda_{2} \in(0.9,1.1)\right\} .
$$

We obtain the following existence result:

Theorem 3.2. (Existence and uniqueness of minimizer: General case). There exist $\ell_{0} \in \mathbb{N}$ and, for each $\ell \geqq \ell_{0}$, an open interval $M^{\ell}$ only depending on $v_{2}, v_{3}$, and $\ell$, with $\mu_{\ell}^{\text {us }} \in M^{\ell}$, such that for all $\mu \in M^{\ell}$ there is a unique pair of bondlengths $\left(\lambda_{1}^{\mu}, \lambda_{2}^{\mu}\right)$ such that $\mathcal{F}_{\lambda_{1}^{\mu}, \lambda_{2}^{\mu}, \mu}$ is a solution of the problem (10).

In the following the minimizer is denoted by $\mathcal{F}_{\mu}^{*}$. Note that we have $\mathcal{F}_{\mu_{\ell}^{\text {us }}}^{*}=\mathcal{G}_{\alpha_{\ell}^{\text {us }}}$ by Proposition 3.1 . 
Our aim is to investigate the local stability of $\mathcal{F}_{\mu}^{*}$. To this end, we consider general small perturbations $\tilde{\mathcal{F}}$ of $\mathcal{F}_{\mu}^{*}$ with the same bond graph, i.e., each atom keeps three and only three bonds, and we can identify the three neighboring atoms of the perturbed configurations with the ones for the configuration $\mathcal{F}_{\mu}^{*}$. By $F_{n}^{\mu}=$ $\left\{x_{1}^{\mu}, \ldots, x_{n}^{\mu}\right\}$ denote the $n$-cell of $\mathcal{F}_{\mu}^{*}$ so that $\mathcal{F}_{\mu}^{*}=\left(F_{n}^{\mu}, L_{m}^{\mu}\right)$ with $L_{m}^{\mu}$ as defined in (7) for $m \in \mathbb{N}$ with $n=4 m \ell$. We define small perturbations $\mathscr{P}_{\eta}(\mu)$ of $\mathcal{F}_{\mu}^{*}$ by

$$
\mathscr{P}_{\eta}(\mu)=\left\{\tilde{\mathcal{F}}=\left(F_{n}, L_{m}^{\mu}\right) \mid F_{n}:=\left\{x_{1}, \ldots, x_{n}\right\} \text { with }\left|x_{i}-x_{i}^{\mu}\right| \leqq \eta\right\} .
$$

The parameter $\eta>0$ will always be chosen sufficiently small such that the topology of the bond graph remains invariant. $\eta$ will in general also depend on $\ell$. Moreover, we recall $E(\tilde{\mathcal{F}})=E\left(F_{n}, L_{m}^{\mu}\right)$. We obtain our main result, concerning local stability under small stretching.

Theorem 3.3. (Local stability of minimizers). There exist $\ell_{0} \in \mathbb{N}$ and for each $\ell \geqq \ell_{0}$ some $\mu_{\ell}^{\text {crit }}>\mu_{\ell}^{\mathrm{us}}$ and $\eta_{\ell}>0$ only depending on $v_{2}, v_{3}$, and $\ell$ such that for all $\ell \geqq \ell_{0}$ and for all $\mu \in\left[\mu_{\ell}^{\mathrm{us}}, \mu_{\ell}^{\text {crit }}\right]$ we have

$$
E(\tilde{\mathcal{F}})>E\left(\mathcal{F}_{\mu}^{*}\right)
$$

for any nontrivial perturbation $\tilde{\mathcal{F}} \in \mathscr{P}_{\eta_{\ell}}(\mu)$ of the configuration $\mathcal{F}_{\mu}^{*}$.

The theorem asserts that, under prescribed and small stretchings (i.e., the value of $L_{m}^{\mu}$ is prescribed), there exists a periodic strict-local minimizer $\mathcal{F}_{\mu}^{*}$ that belongs to the family $\mathscr{F}(\mu)$. In other words, given $\mu>\mu^{\text {us }}$, the $\mu$-periodic configuration $\mathcal{F}_{\mu}^{*}$ is a local minimizer among configurations subject to the same macroscopic stretching, i.e., the atoms follow the macroscopic deformation. This can be seen as a validation of the Cauchy-Born rule in this specific setting. Especially, the result justifies the reduction of the $3 n$-dimensional minimization problem $\min \left\{E(\mathcal{F}) \mid \mathcal{F} \in \mathscr{P}_{\eta_{\ell}}(\mu)\right\}$ to the two-dimensional problem (10).

In the following statement we collect the main properties of the local minimizer:

Proposition 3.4. (Properties of minimizer). There exist $\ell_{0} \in \mathbb{N}$ and for each $\ell \geqq \ell_{0}$ an open interval $M^{\ell}$ only depending on $v_{2}, v_{3}$, and $\ell$, with $\mu_{\ell}^{\mathrm{us}} \in M^{\ell}$, such that:

1. The mapping $\mu \mapsto E\left(\mathcal{F}_{\mu}^{*}\right)=E_{\min }(\mu)$ is smooth, strictly convex on $M^{\ell}$ and attains its minimum in $\mu_{\ell}^{\mathrm{us}}$. Particularly, $\frac{d^{2}}{d \mu^{2}} E_{\min }\left(\mu_{\ell}^{\mathrm{us}}\right) \geqq$ cn for $c>0$ only depending on $v_{2}, v_{3}$.

2. The lengths $\lambda_{1}^{\mu}, \lambda_{2}^{\mu}$ increase continuously for $\mu \in M^{\ell}$. In particular, we have $\lambda_{1}^{\mu}, \lambda_{2}^{\mu}>1$ for $\mu>\mu_{\ell}^{\text {us }}$ and $\lambda_{1}^{\mu}, \lambda_{2}^{\mu}<1$ for $\mu<\mu_{\ell}^{\text {us }}$.

3. The angle $\alpha^{\mu}$ corresponding to $\lambda_{1}^{\mu}, \lambda_{2}^{\mu}$ given by the relations (3) and (5) satisfies $\alpha^{\mu} \in\left(\alpha_{\ell}^{\mathrm{ch}}, \alpha^{\mathrm{ru}}\right)$ for all $\mu \in M^{\ell}$.

4. Whenever $v_{2}^{\prime \prime}(1) \neq 6 v_{3}^{\prime \prime}(2 \pi / 3)$, the radius $\rho^{\mu}$ corresponding to $\lambda_{1}^{\mu}, \lambda_{2}^{\mu}$ given by relation (3) is continuously increasing or decreasing for $\mu \in M^{\ell}$, respectively, depending on whether $v_{2}^{\prime \prime}(1)<6 v_{3}^{\prime \prime}(2 \pi / 3)$ or $v_{2}^{\prime \prime}(1)>6 v_{3}^{\prime \prime}(2 \pi / 3)$. 
Properties 1 and 2 imply that that the nanotubes show elastic response for small extension and compression. Property 3 reconfirms that neither the polyhedral nor the rolled-up configuration is a local minimizer of the energy, for all $\mu$ near $\mu_{\ell}^{\text {us }}$. Finally, Property 4 implies that under stretching or compressing the radius of the nanotube changes whenever $v_{2}^{\prime \prime}(1) \neq 6 v_{3}^{\prime \prime}(2 \pi / 3)$. In particular, if $v_{2}^{\prime \prime}(1)>6 v_{3}^{\prime \prime}(2 \pi / 3)$, the radius of the nanotube decreases as changing the angles is energetically more convenient.

Notice that Theorem 3.3 provides a stability result only for the case of expansion $\mu \geqq \mu_{\ell}^{\text {us }}$ and for values $\mu$ near $\mu_{\ell}^{\text {us }}$. The situation for compression is more subtle from an analytical point of view and our proof techniques do not apply in this case. However, we expect stability of nanotubes also for small compression and refer to [55] for some numerical results in this direction. Let us complete the picture in the tension regime by discussing briefly the fact that for larger stretching cleavage along a section is energetically favored. More precisely, we have

Theorem 3.5. (Fracture). Let $\mathcal{H}_{\mu}$ be the configuration

$$
x_{i, k}^{j, l}= \begin{cases}\bar{x}_{i, k}^{j, l} & j \in[0, m / 2)+m \mathbb{Z}, \\ \bar{x}_{i, k}^{j, l}+m\left(\mu-\mu_{\ell}^{\text {us }}\right) & \text { else }\end{cases}
$$

for $i=1, \ldots, \ell$ and $k, l \in\{0,1\}$, where $\bar{x}_{i, k}^{j, l}$ denote the atomic positions of the

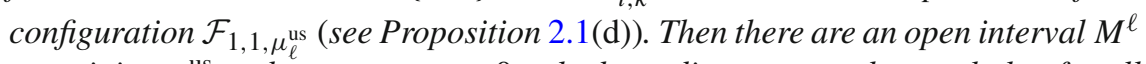
containing $\mu_{\ell}^{\text {us }}$ and a constant $c>0$ only depending on $v_{2}$ and $v_{3}$ such that for all $\mu \in M^{\ell}, \mu \geqq \mu_{\ell, m}^{\mathrm{frac}}:=\mu_{\ell}^{\mathrm{us}}+c / \sqrt{m}$, one has $E\left(\mathcal{H}_{\mu}\right)<E\left(\mathcal{F}_{\mu}^{*}\right)$.

Notice that the configuration $\mathcal{H}_{\mu}$ corresponds to a brittle nanotube cleaved along a cross-section. The energy is given by $E\left(\mathcal{H}_{\mu}\right)=E\left(\mathcal{F}_{1,1, \mu_{\ell}^{\text {us }}}\right)+4 \ell$ since in the con-

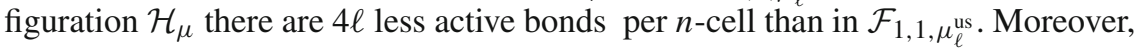
$\mathcal{H}_{\mu}$ is a stable configuration in the sense of Theorem 3.3 for all $\mu \geqq \mu_{\ell}^{\text {us }}$, which can be seen by applying Theorem 3.3 separately on the two parts of $\mathcal{H}_{\mu}$, consisting of the points $x_{i, k}^{j, l}$ with $j<m / 2$ and $j \geqq m / 2$, respectively.

As mentioned, nanotubes are long structures. In particular, $m$ should be expected to be many orders of magnitude larger than $\ell$. The case of large $m$ is hence a sensible one and for $m$ large enough we have $\mu_{\ell, m}^{\text {frac }}<\mu_{\ell}^{\text {crit }}$, with $\mu_{\ell}^{\text {crit }}$ from Theorem 3.3. Hence, by combining Theorem 3.3 with Theorem 3.5 , for all $\mu \geqq \mu_{\ell}^{\text {us }}$ we obtain a stability result for an elastically stretched or cleaved nanotube, respectively.

The proof of Theorem 3.5 is elementary and relies on the fact that the difference of the energy associated to $\mathcal{F}_{\mu}^{*}$ and $\mathcal{H}_{\mu}$ can be expressed by

$$
\begin{aligned}
& E\left(\mathcal{H}_{\mu}\right)-E\left(\mathcal{F}_{\mu}^{*}\right)=4 \ell+E\left(\mathcal{F}_{1,1, \mu_{\ell}^{\text {us }}}\right)-E\left(\mathcal{F}_{\mu}^{*}\right)=4 \ell+E_{\min }\left(\mu_{\ell}^{\text {us }}\right)-E_{\min }(\mu) \\
& =4 \ell-\frac{1}{2} \frac{d^{2}}{d^{2} \mu} E_{\min }\left(\mu_{\ell}^{\mathrm{us}}\right)\left(\mu-\mu_{\ell}^{\mathrm{us}}\right)^{2}+\mathrm{O}\left(\left(\mu-\mu_{\ell}^{\mathrm{us}}\right)^{3}\right) \\
& \leqq 4 \ell-\frac{1}{4} \frac{d^{2}}{d^{2} \mu} E_{\min }\left(\mu_{\ell}^{\mathrm{us}}\right)\left(\mu-\mu_{\ell}^{\mathrm{us}}\right)^{2} \leqq 4 \ell-m \ell c\left(\mu-\mu_{\ell}^{\mathrm{us}}\right)^{2}
\end{aligned}
$$

for $\mu$ in a small neighborhood around $\mu_{\ell}^{\text {us }}$, where we used Property 1 in Proposition 3.4 and $n=4 m \ell$. 


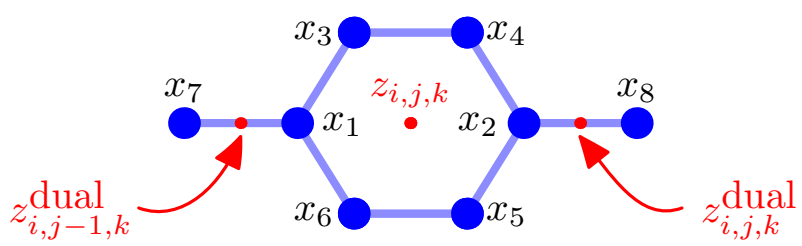

Fig. 5. Notation for the points and the centers in the basic cell

We close the section by noting that the scaling of $\mu_{\ell, m}^{\mathrm{frac}}-\mu_{\ell}^{\mathrm{us}}$ in $m$ is typical for atomistic systems with pairwise interactions of Lennard-Jones type and has also been obtained in related models, cf. [5,33,34].

\section{Existence and Stability: Proof of Theorem 3.2 and Theorem 3.3}

In this section we consider small perturbations $\tilde{\mathcal{F}}$ of configurations in $\mathscr{F}(\mu)$ with the same bond graph, as defined in (11). The atomic positions of $\tilde{\mathcal{F}}$ will be indicated by $x_{i, k}^{j, l}$ and are labeled as for a configuration $\mathscr{F}(\mu)$, cf. Proposition 2.1(d). We first introduce some further notation needed for the proof of our main result. In particular, we introduce a cell energy corresponding to the energy contribution of a specific basic cell.

Centers and dual centers. We introduce the cell centers

$$
z_{i, j, k}=\frac{1}{2}\left(x_{i, k}^{j, 0}+x_{i, k}^{j, 1}\right)
$$

and the dual cell centers

$$
z_{i, j, k}^{\mathrm{dual}}=\frac{1}{2}\left(x_{i, k}^{j, 1}+x_{i, k}^{j+1,0}\right) .
$$

Note that for a configuration in $\mathcal{F}(\mu)$ for fixed $j$ the $2 \ell$ points $z_{i, j, 0}$ and $z_{i, j-1,1}^{\text {dual }}$ for $i=1, \ldots, \ell$ lie in a plane perpendicular to $e_{1}$. Likewise, $z_{i, j, 1}$ and $z_{i, j, 0}^{\text {dual }}$ for $i=1, \ldots, \ell$ lie in a plane perpendicular to $e_{1}$.

Cell energy. The main strategy of our proof will be to reduce the investigation of (10) to a cell problem. In order to correctly capture the contribution of all bond lengths and angles to the energy, it is not enough to consider a hexagon as a basic cell, but two additional atoms have to be taken into account.

Let be given a center $z_{i, j, k}$ and number the atoms of the corresponding hexagon by $x_{1}=x_{i, k}^{j, 0}, x_{2}=x_{i, k}^{j, 1}$ and the remaining clockwisely by $x_{3}, x_{4}, x_{5}, x_{6}$ as indicated in Fig. 5, such that $x_{3}$ is consecutive to $x_{1}$, see also (54) below. Additionally, the atoms bonded to $x_{1}$ and $x_{2}$, respectively, which are not contained in the hexagon, are denoted by $x_{7}$ and $x_{8}$. Note that $z_{i, j-1, k}^{\text {dual }}=\left(x_{7}+x_{1}\right) / 2$ and $z_{i, j, k}^{\text {dual }}=\left(x_{2}+x_{8}\right) / 2$. For $i=1, \ldots, 6$ we define the bondlengths $b_{i}$ as indicated in Fig. 6 and $b_{7}=\left|x_{1}-x_{7}\right|$, $b_{8}=\left|x_{2}-x_{8}\right|$, where

$$
2\left|z_{i, j-1, k}^{\text {dual }}-x_{1}\right|=b_{7}, \quad 2\left|z_{i, j, k}^{\text {dual }}-x_{2}\right|=b_{8} .
$$




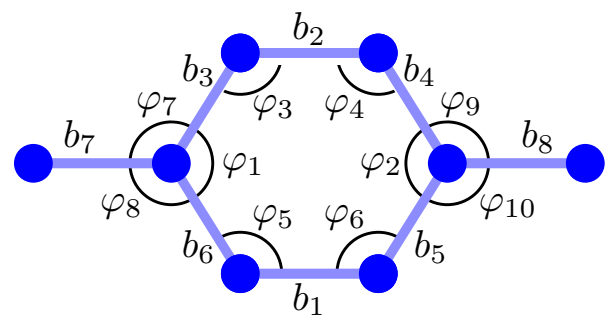

Fig. 6. Notation for the bond lengths and angles in the basic cell

By $\varphi_{i}$ we denote the interior angle of the hexagon at $x_{i}$. By $\varphi_{7}, \varphi_{8}$ we denote the remaining two angles at $x_{1}$ and by $\varphi_{9}, \varphi_{10}$ we denote the remaining two angles at $x_{2}$, see again Fig. 6 .

We define the cell energy by

$$
\begin{aligned}
E_{\text {cell }}\left(z_{i, j, k}\right)= & \frac{1}{4}\left(v_{2}\left(b_{1}\right)+v_{2}\left(b_{2}\right)\right)+\frac{1}{2} \sum_{h=3}^{6} v_{2}\left(b_{h}\right)+\frac{1}{4}\left(v_{2}\left(b_{7}\right)+v_{2}\left(b_{8}\right)\right) \\
& +v_{3}\left(\varphi_{1}\right)+v_{3}\left(\varphi_{2}\right)+\frac{1}{2} \sum_{h=3}^{6} v_{3}\left(\varphi_{h}\right)+\frac{1}{2} \sum_{h=7}^{10} v_{3}\left(\varphi_{h}\right)
\end{aligned}
$$

Notice that the cell energy is a function depending on the bond lengths and angles in the cell. However, as we identify each cell with its center $z_{i, j, k}$, for simplicity we use the notation $E_{\text {cell }}=E_{\text {cell }}\left(z_{i, j, k}\right)$. Furthermore, also for notational convenience we do not put indices $i, j, k$ on bond lengths and angles. To derive convexity properties of $E_{\text {cell }}$ it is convenient to take also the contribution of the angles $\varphi_{7}, \ldots, \varphi_{10}$ into account. Observe that

$$
E(\tilde{\mathcal{F}})=\sum_{i=1}^{\ell} \sum_{j=1}^{m} \sum_{k=0,1} E_{\mathrm{cell}}\left(z_{i, j, k}\right) .
$$

Indeed, each bond not (approximately) parallel to $e_{1}$ is contained exactly in two cells. Each bond (approximately) parallel to $e_{1}$ is contained in four cells, twice in form of a bond in a hexagon, once as a bond left of a hexagon and once as a bond right of a hexagon. Moreover, angles with index $\{1,2\}$ are contained exactly in one cell and angles with index $\{3, \ldots, 10\}$ are contained in exactly two cells.

Symmetrization of cells. A basic cell is a configuration of eight points of $\mathbb{R}^{3}$. By $\boldsymbol{x}_{\text {kink }}^{\ell} \in \mathbb{R}^{3 \times 8}$ we denote the unstretched kink configuration: a basic cell as found in the unstretched configuration $\mathcal{G}_{\alpha_{\ell}^{\text {us }}}$ from Section 3, see (54) below for the exact definition. Notice that the coordinates given in (54) correspond to a convenient choice of a new reference orthonormal system in $\mathbb{R}^{3}$.

Indeed, consider a cell of the nanotube $\mathcal{G}_{\alpha_{\ell}}$, where the eight points are ordered from $x_{1}$ to $x_{8}$ according to the convention of the previous subsection (see Fig. 5), in particular the points $x_{3}, x_{4}, x_{5}, x_{6}$ are numbered clockwisely with respect to an observer lying in the interior of the tube. We fix a new reference coordinate system as follows: we let the center of the cell be the origin, $e_{1}$ (axis direction) be the 
direction of $x_{2}-x_{1}, e_{2}$ the direction of $x_{3}-x_{6}$, and $e_{3}=e_{1} \wedge e_{2}$. Sometimes we will write $\mathbb{R}^{2} \times\{0\}$ for the plane generated by $e_{1}, e_{2}$. If $\boldsymbol{x} \in \mathbb{R}^{3 \times 8}$ denotes a generic cell, possibly after a rigid motion we may always assume that, with respect to the new reference system, the second and third components of $\left(x_{1}+x_{7}\right) / 2,\left(x_{2}+x_{8}\right) / 2$ are zero and the points $x_{4}, x_{5}$ lie in a plane parallel to $\mathbb{R}^{2} \times\{0\}$.

A key step in our analysis will be to show that the minimization of the cell energy (13) can be reduced to a special situation with high symmetry. To this end, we introduce the symmetrization of a cell. For $y=\left(y^{1}, y^{2}, y^{3}\right) \in \mathbb{R}^{3}$ we let $r_{1}(y):=\left(-y^{1}, y^{2}, y^{3}\right)$ and $r_{2}(y):=\left(y^{1},-y^{2}, y^{3}\right)$. For the generic cell $\boldsymbol{x}=$ $\left(x_{1}, \ldots, x_{8}\right) \in \mathbb{R}^{3 \times 8}$ we define the reflections

$$
\begin{aligned}
& S_{1}(\boldsymbol{x})=\left(r_{2}\left(x_{1}\right)\left|r_{2}\left(x_{2}\right)\right| r_{2}\left(x_{6}\right)\left|r_{2}\left(x_{5}\right)\right| r_{2}\left(x_{4}\right)\left|r_{2}\left(x_{3}\right)\right| r_{2}\left(x_{7}\right) \mid r_{2}\left(x_{8}\right)\right), \\
& S_{2}(\boldsymbol{x})=\left(r_{1}\left(x_{2}\right)\left|r_{1}\left(x_{1}\right)\right| r_{1}\left(x_{4}\right)\left|r_{1}\left(x_{3}\right)\right| r_{1}\left(x_{6}\right)\left|r_{1}\left(x_{5}\right)\right| r_{1}\left(x_{8}\right) \mid r_{1}\left(x_{7}\right)\right) .
\end{aligned}
$$

$S_{1}$ interchanges the pair of points $\left(x_{3}, x_{6}\right)$ and $\left(x_{4}, x_{5}\right)$, and changes the sign of the second components of all points. On the other hand, $S_{2}$ interchanges the pair of points $\left(x_{1}, x_{2}\right),\left(x_{3}, x_{4}\right),\left(x_{5}, x_{6}\right)$, and $\left(x_{7}, x_{8}\right)$, and changes the sign of the first components of all points.

We let

$$
\boldsymbol{x}_{S_{1}}:=\boldsymbol{x}_{\mathrm{kink}}^{\ell}+S_{1}\left(\boldsymbol{x}-\boldsymbol{x}_{\mathrm{kink}}^{\ell}\right), \quad \boldsymbol{x}_{S_{2}}:=\boldsymbol{x}_{\mathrm{kink}}^{\ell}+S_{2}\left(\boldsymbol{x}-\boldsymbol{x}_{\mathrm{kink}}^{\ell}\right) .
$$

If $\boldsymbol{x}$ is seen as a perturbation of $\boldsymbol{x}_{\text {kink }}^{\ell}, \boldsymbol{x}_{S_{1}}$ (resp. $\boldsymbol{x}_{S_{2}}$ ) is the reflected perturbation with respect to the plane generated by $e_{1}, e_{3}$ (resp. $\left.e_{2}, e_{3}\right)$. The symmetry of the configurations implies therefore $E_{\text {cell }}\left(\boldsymbol{x}_{S_{2}}\right)=E_{\text {cell }}\left(\boldsymbol{x}_{S_{1}}\right)=E_{\text {cell }}(\boldsymbol{x})$.

We define the symmetrized perturbations

$$
\begin{aligned}
\boldsymbol{x}^{\prime} & :=\boldsymbol{x}_{\mathrm{kink}}^{\ell}+\frac{1}{2}\left(\left(\boldsymbol{x}-\boldsymbol{x}_{\mathrm{kink}}^{\ell}\right)+S_{1}\left(\boldsymbol{x}-\boldsymbol{x}_{\mathrm{kink}}^{\ell}\right)\right), \\
\mathcal{S}(\boldsymbol{x}) & :=\boldsymbol{x}_{\mathrm{kink}}^{\ell}+\frac{1}{2}\left(\left(\boldsymbol{x}^{\prime}-\boldsymbol{x}_{\mathrm{kink}}^{\ell}\right)+S_{2}\left(\boldsymbol{x}^{\prime}-\boldsymbol{x}_{\mathrm{kink}}^{\ell}\right)\right) .
\end{aligned}
$$

We also introduce the symmetry defect

$$
\Delta\left(z_{i, j, k}\right):=\left|\boldsymbol{x}-\boldsymbol{x}^{\prime}\right|^{2}+\left|\boldsymbol{x}^{\prime}-\mathcal{S}(\boldsymbol{x})\right|^{2} .
$$

Notice that for notational simplicity in (18) we do not put indices $i, j, k$ on $\boldsymbol{x}, \boldsymbol{x}^{\prime}$, and $\mathcal{S}(\boldsymbol{x})$. A property that we remark is that for a basic cell $\boldsymbol{x}$ with center $z_{i, j, k}$ the quantity $\left|z_{i, j, k}^{\text {dual }}-z_{i, j-1, k}^{\text {dual }}\right|$ does not change when passing to $\mathcal{S}(\boldsymbol{x})$ since the second and third component of $z_{i, j, k}^{\text {dual }}, z_{i, j-1, k}^{\text {dual }}$ are assumed to be zero. Below we will see that the difference of the cell energy of $\mathcal{S}(\boldsymbol{x})$ and $\boldsymbol{x}$ can be controlled in terms of $\Delta\left(z_{i, j, k}\right)$ due to strict convexity of the energy.

Angles between planes. In what follows we denote the plane through three points $p_{1}, p_{2}$, and $p_{3}$ by $\left\{p_{1} p_{2} p_{3}\right\}$, i.e.,

$$
\left\{p_{1} p_{2} p_{3}\right\}:=\operatorname{span}_{\mathbb{R}}\left\{p_{1}-p_{2}, p_{3}-p_{2}\right\} .
$$

Furthermore, for each $y=x_{i, k}^{j, l}$ we denote by $y_{1}, y_{2}, y_{3}$ the three atoms that are bonded with $y$, where the three points are numbered such that $y_{3}-y$ is (approximately) parallel to the axis direction $e_{1}$. Let $\theta=\theta(x) \leqq \pi$ denote the angle 


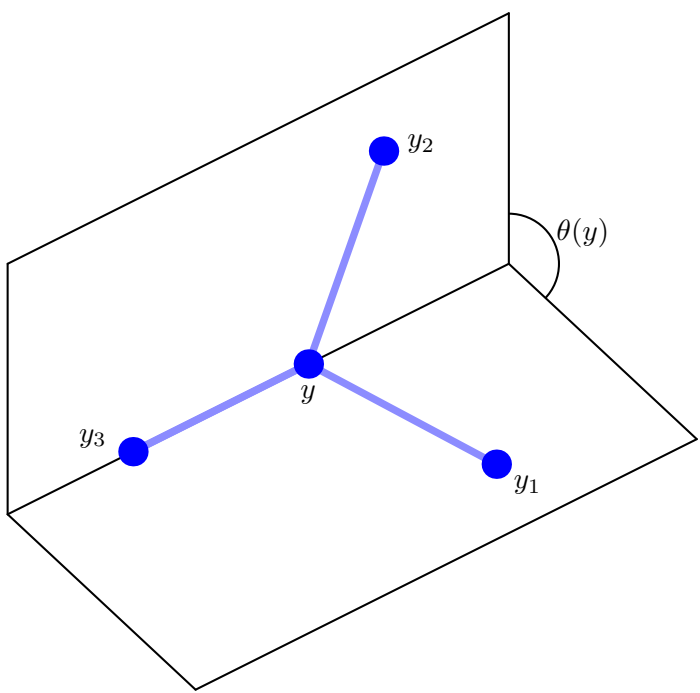

Fig. 7. The angle between the planes $\left\{y_{3} y y_{1}\right\}$ and $\left\{y_{3} y y_{2}\right\}$ is denoted by $\theta(y)$

between the planes defined by $\left\{y_{3} y y_{1}\right\}$ and $\left\{y_{3} y y_{2}\right\}$. More precisely, let $n_{13}, n_{23}$ denote unit normal vectors to the planes $\left\{y_{3} y y_{1}\right\}$ and $\left\{y_{3} y y_{2}\right\}$, respectively. Then we have

$$
\theta(y)=\max \left\{\pi-\arccos \left(n_{13} \cdot n_{23}\right), \arccos \left(n_{13} \cdot n_{23}\right)\right\}
$$

as represented in Fig. 7. With these preparations we will now define angles corresponding to centers and dual centers. Let $z_{i, j, k}=\frac{1}{2}\left(x_{i, k}^{j, 0}+x_{i, k}^{j, 1}\right)$ be a center of a given hexagon. As before we denote the points of the hexagon by $x_{1}, \ldots, x_{6}$. By $\theta_{l}\left(z_{i, j, k}\right)$ we denote the angle between the planes $\left\{x_{1} x_{3} x_{4}\right\}$ and $\left\{x_{1} x_{6} x_{5}\right\}$. By $\theta_{r}\left(z_{i, j, k}\right)$ we denote the angle between the planes $\left\{x_{3} x_{4} x_{2}\right\}$ and $\left\{x_{2} x_{5} x_{6}\right\}$. For a dual center $z_{i, j, k}^{\text {dual }}=\left(x_{i, k}^{j, 1}+x_{i, k}^{j+1,0}\right) / 2$ we introduce $\theta_{l}\left(z_{i, j, k}^{\text {dual }}\right)=\theta\left(x_{i, k}^{j, 1}\right)$ and $\theta_{r}\left(z_{i, j, k}^{\text {dual }}\right)=\theta\left(x_{i, k}^{j+1,0}\right)$.

In Section 5 we prove the following lemma which provides a linear control for the oscillation of plane angles of a perturbed configuration $\tilde{\mathcal{F}}$ with respect to those of a configuration in $\mathscr{F}(\mu)$ in terms of the symmetry defect from (18).

Lemma 4.1. (Symmetry defect controls angle defect). There is a universal constant $c>0$ such that for $\eta>0$ small enough for all $\tilde{\mathcal{F}} \in \mathscr{P}_{\eta}(\mu)$ with $\Delta\left(z_{i, j, k}\right) \leqq \eta$ for all centers $z_{i, j, k}$ we have

$$
\begin{aligned}
& \sum_{j=1}^{m} \sum_{i=1}^{\ell} \sum_{k=0,1}\left(\theta_{l}\left(z_{i, j, k}\right)+\theta_{l}\left(z_{i, j, k}^{\text {dual }}\right)+\theta_{r}\left(z_{i, j, k}\right)+\theta_{r}\left(z_{i, j, k}^{\text {dual }}\right)\right) \\
& \leqq 4 m(2 \ell-2) \pi+c \sum_{j=1}^{m} \sum_{i=1}^{\ell} \sum_{k=0,1} \Delta\left(z_{i, j, k}\right) .
\end{aligned}
$$




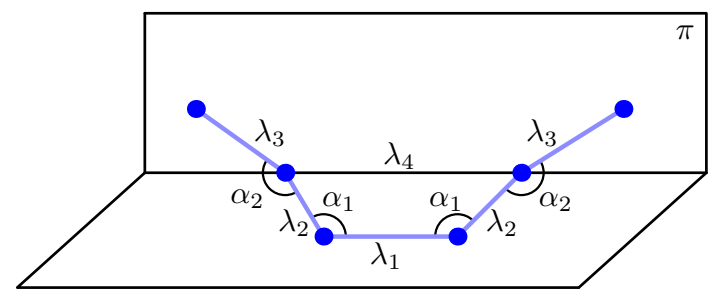

Fig. 8. Half of a cell configuration kinked at the plane $\pi$ and satisfying conditions (20). The other half of the cell configuration can be determined by symmetry with respect to the plane $\pi$

Note that the sum on the left equals exactly $4 m(2 \ell-2) \pi$ if $\tilde{\mathcal{F}} \in \mathscr{F}(\mu)$.

Reduced energy. A key step in our analysis will be to show that the minimization of the cell energy (13) can be reduced to a special situation with high symmetry. As represented in Fig. 8, this corresponds to the conditions

$$
\begin{aligned}
& b_{1}=b_{2}=\lambda_{1}, \quad b_{3}=b_{4}=b_{5}=b_{6}=\lambda_{2}, \quad b_{7}=b_{8}=\lambda_{3}, \\
& z_{i, j, k}^{\text {dual }}-z_{i, j-1, k}^{\text {dual }}=\tilde{\mu} e_{1}, \quad x_{2}-x_{1}=\lambda_{4} e_{1}, \\
& \varphi_{1}=\varphi_{2}=\beta, \quad \varphi_{3}=\varphi_{4}=\varphi_{5}=\varphi_{6}=\alpha_{1}, \quad \varphi_{7}=\varphi_{8}=\varphi_{9}=\varphi_{10}=\alpha_{2}, \\
& \theta_{l}\left(z_{i, j, k}\right)=\theta_{r}\left(z_{i, j, k}\right)=\gamma_{1}, \quad \theta_{l}\left(z_{i, j, k}^{\text {dual }}\right)=\theta_{r}\left(z_{i, j-1, k}^{\text {dual }}\right)=\gamma_{2}
\end{aligned}
$$

with $\lambda_{1}, \lambda_{2}, \lambda_{3} \in(0.9,1.1), \lambda_{4} \in(0.9,3.3), \tilde{\mu} \in(2.6,3.1), \alpha_{1}, \alpha_{2}, \beta \in$ $(\arccos (-0.4), \arccos (-0.6)), \gamma_{1}, \gamma_{2} \in\left[\frac{3}{4} \pi, \pi\right]$. Note that $\theta_{r}\left(z_{i, j-1, k}^{\text {dual }}\right)=\theta\left(x_{1}\right)$ and $\theta_{l}\left(z_{i, j, k}^{\text {dual }}\right)=\theta\left(x_{2}\right)$ with the angles introduced in (19). The notation $\tilde{\mu}$ is reminiscent of the fact that we have indeed $\tilde{\mu}=\mu$ for a basic cell of a nanotube in $\mathscr{F}(\mu)$. Under (20), arguing along the lines of Proposition 2.2, we obtain

$\beta=\beta\left(\alpha_{1}, \gamma_{1}\right)=2 \arcsin \left(\sin \alpha_{1} \sin \frac{\gamma_{1}}{2}\right)=\beta\left(\alpha_{2}, \gamma_{2}\right)=2 \arcsin \left(\sin \alpha_{2} \sin \frac{\gamma_{2}}{2}\right)$.

By elementary trigonometry, cf. Fig. 8, we also get

$$
\lambda_{4}=\lambda_{1}-2 \lambda_{2} \cos \alpha_{1} .
$$

We now introduce the symmetric energy by

$$
\begin{aligned}
E_{\mu, \gamma_{1}, \gamma_{2}}^{\mathrm{sym}}\left(\lambda, \alpha_{1}, \alpha_{2}\right)= & 2 v_{2}(\lambda)+\frac{1}{2} v_{2}\left(\mu / 2+\lambda \cos \alpha_{1}\right)+\frac{1}{2} v_{2}\left(\mu / 2+\lambda \cos \alpha_{2}\right) \\
& +2 v_{3}\left(\alpha_{1}\right)+2 v_{3}\left(\alpha_{2}\right)+v_{3}\left(\beta\left(\alpha_{1}, \gamma_{1}\right)\right)+v_{3}\left(\beta\left(\alpha_{2}, \gamma_{2}\right)\right) .
\end{aligned}
$$

Notice that $E_{\text {cell }}\left(z_{i, j, k}\right)=E_{\widetilde{\mu}, \gamma_{1}, \gamma_{2}}^{\mathrm{sym}}\left(\lambda, \alpha_{1}, \alpha_{2}\right)$ if the conditions (20) hold with $\alpha_{1}=\alpha_{2}, \gamma_{1}=\gamma_{2}, \lambda_{1}=\lambda_{3}=\mu / 2+\lambda \cos \alpha_{1}$, and $\lambda_{2}=\lambda$. In general, we show that, up to a small perturbation, the symmetric energy $E_{\widetilde{\mu}, \gamma_{1}, \gamma_{2}}^{\mathrm{sym}}$ delivers a lower bound for $E_{\text {cell }}$ for cells satysfying (20). 
Lemma 4.2. (Cell energy and symmetric energy). There exist a constant $c_{0}>0$ and $\ell_{0} \in \mathbb{N}$ only depending on $v_{2}$ and $v_{3}$ such that for each $\tilde{\mathcal{F}} \in \mathscr{P}_{\eta}(\mu)$ and all centers $z_{i, j, k}$ satisfying conditions $(20)$ with $\left|\lambda_{1}-1\right|+\left|\lambda_{3}-1\right| \leqq \ell^{-4}$ and $\left|\gamma_{1}-\gamma_{2}\right| \leqq \ell^{-2}$ we have

$$
E_{\text {cell }}\left(z_{i, j, k}\right) \geqq E_{\widetilde{\mu}, \gamma_{1}, \gamma_{2}}^{\mathrm{sym}}\left(\lambda_{2}, \alpha_{1}, \alpha_{2}\right)-c_{0} \ell^{-4}\left(\gamma_{1}-\gamma_{2}\right)^{2} .
$$

This lemma will be proved in Section 6. The idea in the proof is to express $\lambda_{3}$ in terms of the relations (20) and (22) to find $\lambda_{3}=\tilde{\mu}-\lambda_{1}+2 \lambda \cos \alpha_{1}+\mathrm{O}\left(\left(\gamma_{1}-\gamma_{2}\right)^{2}\right)$, where we set $\lambda=\lambda_{2}$. Here the term $\mathrm{O}\left(\left(\gamma_{1}-\gamma_{2}\right)^{2}\right)$ appears as the points $x_{7}, x_{1}, x_{2}, x_{8}$ in general do not lie on a line. Likewise, we obtain $\lambda_{1}=\tilde{\mu}-\lambda_{3}+2 \lambda \cos \alpha_{2}+$ $\mathrm{O}\left(\left(\gamma_{1}-\gamma_{2}\right)^{2}\right)$. Finally, we use $v_{2}\left(\lambda_{1}\right)+v_{2}\left(\lambda_{3}\right) \geqq 2 v_{2}\left(\left(\lambda_{1}+\lambda_{3}\right) / 2\right)$ by convexity of $v_{2}$.

We also introduce the reduced energy

$$
\begin{aligned}
E_{\mathrm{red}}\left(\mu, \gamma_{1}, \gamma_{2}\right) & =\min \left\{E_{\mu, \gamma_{1}, \gamma_{2}}^{\mathrm{sym}}\left(\lambda, \alpha_{1}, \alpha_{2}\right) \mid \lambda\right. \\
& \left.\in(0.9,1.1), \alpha_{1}, \alpha_{2} \in(\arccos (-0.4), \arccos (-0.6))\right\}
\end{aligned}
$$

Since $E_{\mu, \gamma_{1}, \gamma_{2}}^{\mathrm{sym}}$ is symmetric in $\left(\alpha_{1}, \gamma_{1}\right)$ and $\left(\alpha_{2}, \gamma_{2}\right)$, we observe that $E_{\text {red }}$ is symmetric in $\gamma_{1}$ and $\gamma_{2}$, i.e., $E_{\text {red }}\left(\mu, \gamma_{1}, \gamma_{2}\right)=E_{\text {red }}\left(\mu, \gamma_{2}, \gamma_{1}\right)$. The following result, which is proved in Section 6, collects the fundamental properties of $E_{\text {red }}$.

Proposition 4.3. (Properties of $E_{\mathrm{red}}$ ). There exists $\ell_{0} \in \mathbb{N}$ and for each $\ell \geqq \ell_{0}$ there are open intervals $M^{\ell}, G^{\ell}$ only depending on $v_{2}, v_{3}$ and $\ell$ with $\mu_{\ell}^{\mathrm{us}} \in M^{\ell}$, $\gamma_{\ell} \in G^{\ell}$ (where we recall that $\mu_{\ell}^{\mathrm{us}}$ and $\gamma_{\ell}$ were defined in (9) and (4), respectively) such that the following holds:

1. (Unique minimizer) For each $\left(\mu, \gamma_{1}, \gamma_{2}\right) \in M^{\ell} \times G^{\ell} \times G^{\ell}$ there exists a unique triple $\left(\lambda^{\mu}, \alpha_{1}^{\mu}, \alpha_{2}^{\mu}\right)$ solving the minimization problem (24). Moreover, $\alpha_{1}^{\mu}=\alpha_{2}^{\mu}$ if $\gamma_{1}=\gamma_{2}$. (For simplicity, the dependence of the triple on $\gamma_{1}, \gamma_{2}$ is not included in the notation.)

2. (Strict convexity) $E_{\mathrm{red}}$ is strictly convex on $M^{\ell} \times G^{\ell} \times G^{\ell}$, in particular there is a constant $c_{0}^{\prime}>0$ only depending on $v_{2}$ and $v_{3}$ such that

$$
E_{\text {red }}\left(\mu, \gamma_{1}, \gamma_{2}\right) \geqq E_{\text {red }}(\mu, \bar{\gamma}, \bar{\gamma})+c_{0}^{\prime} \ell^{-2}\left(\gamma_{1}-\gamma_{2}\right)^{2}
$$

with $\bar{\gamma}=\left(\gamma_{1}+\gamma_{2}\right) / 2$ for all $\mu \in M^{\ell}$ and $\gamma_{1}, \gamma_{2} \in G^{\ell}$.

3. (Monotonicity in $\gamma$ ) For each $\mu \in M^{\ell}$, the mapping $g(\gamma):=E_{\mathrm{red}}(\mu, \gamma, \gamma)$ is decreasing on $G^{\ell}$ with $\left|g^{\prime}(\gamma)\right| \leqq C \ell^{-3}$ for all $\gamma \in G^{\ell}$ for some $C>0$ depending only on $v_{3}$.

4. (Monotonicity in $\mu$ ) The mapping $h(\mu):=E_{\mathrm{red}}\left(\mu, \gamma_{\ell}, \gamma_{\ell}\right)$ is strictly convex on $M^{\ell}$ with $h^{\prime \prime}\left(\mu_{\ell}^{\text {us }}\right)>0$ and strictly increasing on $M^{\ell} \cap\left\{\mu \geqq \mu_{\ell}^{\text {us }}\right\}$.

5. (Minimization) For each $\mu \in M^{\ell}$ and $\gamma_{1}=\gamma_{2}=\gamma_{\ell}$, letting $\lambda_{1}^{\mu}=\mu / 2+$ $\lambda^{\mu} \cos \alpha_{1}^{\mu}$ and $\lambda_{2}^{\mu}=\lambda^{\mu}$ with $\lambda^{\mu}$ and $\alpha_{1}^{\mu}$ from 1., the configuration $\mathcal{F}_{\lambda_{1}^{\mu}, \lambda_{2}^{\mu}, \mu}$ is the unique minimizer of the problem (10) with

$$
E\left(\mathcal{F}_{\mu}^{*}\right)=E\left(\mathcal{F}_{\lambda_{1}^{\mu}, \lambda_{2}^{\mu}, \mu}\right)=2 m \ell E_{\text {red }}\left(\mu, \gamma_{\ell}, \gamma_{\ell}\right)
$$


Proof of Theorem 3.2 and Theorem 3.3. We postpone the proofs of the auxiliary results Lemma 4.1, Lemma 4.2, and Proposition 4.3 to the next sections and now proceed with the proof of Theorem 3.2 and Theorem 3.3. For the proof of Proposition 3.4 we refer to Section 6. Moving from the properties of the reduced energy $E_{\text {red }}$, we directly obtain Theorem 3.2.

Proof of Theorem 3.2. Theorem 3.2 follows from Property 5 of Proposition 4.3.

We denote the unique minimzer again by $\mathcal{F}_{\mu}^{*}$ and recall the definition of small perturbations $\mathscr{P}_{\eta}(\mu)$ in (11). Based on the properties of the reduced energy $E_{\text {red }}$, we are able to show that, up to a linear perturbation in terms of the symmetry defect $\Delta$ defined in (18), $E_{\text {red }}$ bounds the cell energy $E_{\text {cell }}$ from below. More precisely, we have the following:

Theorem 4.4. (Energy defect controls symmetry defect). There exist $C>0$ and $\ell_{0} \in \mathbb{N}$ only depending on $v_{2}$ and $v_{3}$, and for each $\ell \geqq \ell_{0}$ there are $\eta_{\ell}>0$ and an open interval $M^{\ell}$ containing $\mu_{\ell}^{\mathrm{us}}$ such that for all $\mu \in M^{\ell}, \tilde{\mathcal{F}} \in \mathscr{P}_{\eta_{\ell}}(\mu)$, and centers $z_{i, j, k}$ we have

$$
E_{\text {cell }}\left(z_{i, j, k}\right) \geqq E_{\text {red }}\left(\left|z_{i, j, k}^{\text {dual }}-z_{i, j-1, k}^{\text {dual }}\right|, \bar{\theta}\left(z_{i, j, k}\right), \bar{\theta}\left(z_{i, j, k}\right)\right)+C \ell^{-2} \Delta\left(z_{i, j, k}\right),
$$

where $\bar{\theta}\left(z_{i, j, k}\right):=\left(\theta_{l}\left(z_{i, j, k}\right)+\theta_{r}\left(z_{i, j, k}\right)+\theta_{l}\left(z_{i, j, k}^{\text {dual }}\right)+\theta_{r}\left(z_{i, j-1, k}^{\text {dual }}\right)\right) / 4$.

We postpone the proof of Theorem 4.4 to Section 7 and close this section with the proof of our main stability result Theorem 3.3.

Proof of Theorem 3.3. Let $M^{\ell}$ be an open interval containing $\mu_{\ell}^{\text {us }}$ such that Proposition 4.3 and Theorem 4.4 hold for all $\mu \in M^{\ell}$ and let $G^{\ell}$ be the interval from Proposition 4.3. Then choose $\mu_{\ell}^{\text {crit }}>\mu_{\ell}^{\text {us }}$ such that $\left[\mu_{\ell}^{\text {us }}, \mu_{\ell}^{\text {crit }}\right] \subset \subset M^{\ell}$. Let $\ell \geqq \ell_{0}$ and $\mu \in\left[\mu_{\ell}^{\text {us }}, \mu_{\ell}^{\text {crit }}\right]$ be given. Consider a nontrivial perturbation $\tilde{\mathcal{F}} \in \mathscr{P}_{\eta_{\ell}}(\mu)$ with $\eta_{\ell}$ as in Theorem 4.4. We denote the atomic positions by $x_{i, k}^{j, l}$ and the centers by $z_{i, j, k}, z_{i, j, k}^{\text {dual }}$ as introduced at the beginning of the section, see (12) and Fig. 5. Define

$$
\bar{\theta}\left(z_{i, j, k}\right)=\frac{1}{4}\left(\theta_{l}\left(z_{i, j, k}\right)+\theta_{r}\left(z_{i, j, k}\right)+\theta_{l}\left(z_{i, j, k}^{\text {dual }}\right)+\theta_{r}\left(z_{i, j-1, k}^{\text {dual }}\right)\right)
$$

and also

$$
\bar{\mu}=\frac{1}{2 m \ell} \sum_{j=1}^{m} \sum_{i=1}^{\ell} \sum_{k=0,1}\left|z_{i, j, k}^{\mathrm{dual}}-z_{i, j-1, k}^{\mathrm{dual}}\right|, \quad \bar{\theta}=\frac{1}{2 m \ell} \sum_{j=1}^{m} \sum_{i=1}^{\ell} \sum_{k=0,1} \bar{\theta}\left(z_{i, j, k}\right) .
$$

Possibly passing to a smaller $\eta_{\ell}$, we get $\left|z_{i, j, k}^{\text {dual }}-z_{i, j-1, k}^{\text {dual }}\right| \in M^{\ell}$ and $\bar{\theta}\left(z_{i, j, k}\right) \in G^{\ell}$ for all $i, j, k$. By Theorem 4.4 we have for each cell

$$
E_{\text {cell }}\left(z_{i, j, k}\right) \geqq E_{\text {red }}\left(\left|z_{i, j, k}^{\text {dual }}-z_{i, j-1, k}^{\text {dual }}\right|, \bar{\theta}\left(z_{i, j, k}\right), \bar{\theta}\left(z_{i, j, k}\right)\right)+C \ell^{-2} \Delta\left(z_{i, j, k}\right)
$$


if $\ell_{0}$ is chosen sufficiently large. Then, taking the sum over all cells and using Property 2. of Proposition 4.3, we get by (14)

$$
\begin{aligned}
E(\tilde{\mathcal{F}})= & \sum_{i=1}^{\ell} \sum_{j=1}^{m} \sum_{k=0,1} E_{\text {cell }}\left(z_{i, j, k}\right) \geqq 2 m \ell E_{\text {red }}(\bar{\mu}, \bar{\theta}, \bar{\theta}) \\
& +C \ell^{-2} \sum_{i=1}^{\ell} \sum_{j=1}^{m} \sum_{k=0,1} \Delta\left(z_{i, j, k}\right) .
\end{aligned}
$$

Possibly passing to a smaller $\eta_{\ell}$, we can assume that $\Delta\left(z_{i, j, k}\right) \leqq \eta$ for all centers with $\eta$ from Lemma 4.1. Then using Lemma 4.1 and recalling (25) we find that

$$
\begin{aligned}
\bar{\theta} & \leqq \frac{1}{8 m \ell}\left(4 m(2 \ell-2) \pi+C \sum_{j=1}^{m} \sum_{i=1}^{\ell} \sum_{k=0,1} \Delta\left(z_{i, j, k}\right)\right) \\
& \leqq \gamma_{\ell}+\frac{c}{2 m \ell} \sum_{j=1}^{m} \sum_{i=1}^{\ell} \sum_{k=0,1} \Delta\left(z_{i, j, k}\right),
\end{aligned}
$$

where in the last step we have used the fact that $\gamma_{\ell}=\pi(1-1 / \ell)$, see (4). This together with Property 3 of Proposition 4.3 yields

$$
E(\tilde{\mathcal{F}}) \geqq 2 m \ell E_{\text {red }}\left(\bar{\mu}, \gamma_{\ell}, \gamma_{\ell}\right)+\left(C \ell^{-2}-C^{\prime} \ell^{-3}\right) \sum_{j=1}^{m} \sum_{i=1}^{\ell} \sum_{k=0,1} \Delta\left(z_{i, j, k}\right)
$$

for some $C^{\prime}>0$ only depending on $v_{3}$. Recalling the constraint in definition (11), we get for fixed $i$ and $k$ that

$$
m \mu=L_{m}^{\mu}=\left|\sum_{j=1}^{m} z_{i, j, k}^{\text {dual }}-z_{i, j-1, k}^{\text {dual }}\right| \leqq \sum_{j=1}^{m}\left|z_{i, j, k}^{\text {dual }}-z_{i, j-1, k}^{\text {dual }}\right|
$$

and therefore, by taking the sum over all $i$ and $k$, we get $\bar{\mu} \geqq \mu \geqq \mu_{\ell}^{\text {us }}$. Then we derive by Property 4 and 5 of Proposition 4.3

$$
\begin{aligned}
E(\tilde{\mathcal{F}}) & \geqq 2 m \ell E_{\text {red }}\left(\mu, \gamma_{\ell}, \gamma_{\ell}\right)+C^{\prime \prime} \ell^{-2} \sum_{i=1}^{\ell} \sum_{j=1}^{m} \sum_{k=0,1} \Delta\left(z_{i, j, k}\right) \\
& =E\left(\mathcal{F}_{\mu}^{*}\right)+C^{\prime \prime} \ell^{-2} \sum_{i=1}^{\ell} \sum_{j=1}^{m} \sum_{k=0,1} \Delta\left(z_{i, j, k}\right)
\end{aligned}
$$

for $\ell_{0}$ sufficiently large and a possibly smaller constant $C^{\prime \prime}>0$. Note that in this step of the proof we have fundamentally used that $\mu \geqq \mu_{\ell}^{\text {us }}$, i.e., the nanotube is stretched, so that a monotonicity argument can be applied.

It remains to confirm the strict inequality $E(\tilde{\mathcal{F}})>E\left(\mathcal{F}_{\mu}^{*}\right)$. If $\Delta\left(z_{i, j, k}\right)>0$ for some center $z_{i, j, k}$, this follows directly from the previous estimate. Otherwise, as $\tilde{\mathcal{F}}$ is a nontrivial perturbation, one of the angles in (25) or one of the lengths 
$\left|z_{i, j, k}^{\text {dual }}-z_{i, j-1, k}^{\text {dual }}\right|$ does not coincide with the corresponding mean value and then at least one of the inequalities (26)-(27) is strict due to the strict convexity and monotonicity of the mappings considered in Proposition 4.3.

\section{Symmetry Defect Controls Angle Defect: Proof of Lemma 4.1}

This short section is devoted to the proof of Lemma 4.1. Recall the definition of the centers in (12), the angles (19), and the symmetry defect (18).

Proof of Lemma 4.1. Let $\tilde{\mathcal{F}}$ be a small perturbation of $\mathcal{F}^{\prime} \in \mathscr{F}(\mu)$, with $\Delta\left(z_{i, j, k}\right) \leqq \eta$ for all centers $z_{i, j, k}$. Due to the symmetry of the problem it suffices to show

$$
\sum_{j=1}^{m} \sum_{i=1}^{\ell}\left(\theta_{l}\left(z_{i, j, 0}\right)+\theta_{l}\left(z_{i, j-1,1}^{\text {dual }}\right)\right) \leqq m(2 \ell-2) \pi+c \sum_{j=1}^{m} \sum_{i=1}^{\ell} \sum_{k=0,1} \Delta\left(z_{i, j, k}\right) .
$$

For brevity we write $\theta_{i}^{\prime}=\theta_{l}\left(z_{\frac{i+1}{2}, j, 0}\right)$ for $i=1,3, \ldots, 2 \ell-1$ and $\theta_{i}^{\prime}=\theta_{l}\left(z_{\frac{i}{2}, j-1,1}^{\text {dual }}\right)$ for $i=2,4, \ldots, 2 \ell$. (Note that for convenience we do not include the index $j$ in the notation.)

Let $n_{i}, n_{i+1}$ be unit normal vectors as introduced before (19) such that $n_{i} \cdot n_{i+1}$ is near 1 and the smallest angle between them, which we denote by $\varangle\left(n_{i}, n_{i+1}\right)$, is given by

$$
\varangle\left(n_{i}, n_{i+1}\right)=\pi-\theta_{i}^{\prime}
$$

for $i=1,3, \ldots, 2 \ell-1$. For a suitable ordering of $n_{i}$ and $n_{i+1}$ we then also obtain $\varangle\left(n_{i}, n_{i+1}\right)=\pi-\theta_{i}^{\prime}$ for $i=2,4, \ldots, 2 \ell$. Fix a center $x_{0} \in \mathbb{R}^{3}$ and let $P$ be the $2 \ell$-gon with vertices $v_{i}:=x_{0}+n_{i}, i=1, \ldots, 2 \ell$. Denote the interior angles accordingly by $\varphi_{i}$. Note that each edge of $P$ forms a triangle with $x_{0}$ with angles $\pi-\theta_{i}^{\prime}, \psi_{i}^{1}$, and $\psi_{i}^{2}$, where $\psi_{i}^{1}$ is the angle at the vertex $v_{i}$ and $\psi_{i}^{2}$ is the angle at $v_{i+1}$. The key ingredient in the proof is now the observation that there exists a universal $c>0$ such that

$$
\begin{aligned}
& \psi_{i+1}^{1}+\psi_{i}^{2}-\varphi_{i+1} \leqq c \Delta\left(z_{\frac{i+1}{2}, j, 0}\right)+c \Delta\left(z_{\frac{i+3}{2}, j, 0}\right) \\
& \psi_{i}^{1}+\psi_{i-1}^{2}-\varphi_{i} \leqq c \Delta\left(z_{\frac{i-1}{2}, j, 0}\right)+c \Delta\left(z_{\frac{i+1}{2}, j, 0}\right)
\end{aligned}
$$

for $i=1,3 \ldots, 2 \ell-1$, where it is understood that $\psi_{0}^{2}=\psi_{2 \ell}^{2}$ and $z_{0, j, 0}=z_{\ell, j, 0}$. We defer the derivation of this property to the end of the proof. Notice that $\theta_{i}^{\prime}=\psi_{i}^{1}+\psi_{i}^{2}$ for $i=1, \ldots, 2 \ell$ and that $\sum_{i=1}^{2 \ell} \varphi_{i} \leqq(2 \ell-2) \pi$ since $P$ is a $2 \ell$-gon. We now obtain by (28)

$$
\sum_{i=1}^{2 \ell} \theta_{i}^{\prime}=\sum_{i=1}^{2 \ell}\left(\psi_{i}^{1}+\psi_{i}^{2}\right) \leqq(2 \ell-2) \pi+c \sum_{i=1}^{\ell} \Delta\left(z_{i, j, 0}\right)
$$

The assertion then follows by taking the sum over all $j=1, \ldots, m$. 
It remains to confirm (28). Fix $i=1,3, \ldots, 2 \ell-1$ and let $N_{i+1}$ be the plane containing the points $v_{i}, v_{i+1}$, and $v_{i+2}$. By $d_{i+1}$ we denote the distance of $x_{0}$ from $N_{i+1}$ and by $n_{i+1}^{\prime}$ the orthogonal projection of the vector $n_{i+1}$ onto $N_{i+1}$. Note that $d_{i+1} \leqq \delta$ for $\delta$ small, depending only on the choice of $\eta$, and that $\left|n_{i+1}^{\prime}\right|=\left|n_{i+1}\right|+\mathrm{O}\left(d_{i+1}^{2}\right)$. The segments $v_{i+2}-v_{i+1}, n_{i+1}^{\prime}$ and $v_{i}-v_{i+1}, n_{i+1}^{\prime}$ enclose two angles, denoted by $\hat{\psi}_{i+1}^{1}$ and $\hat{\psi}_{i}^{2}$, so that $\varphi_{i+1}=\hat{\psi}_{i+1}^{1}+\hat{\psi}_{i}^{2}$. Observe that $\hat{\psi}_{i+1}^{1}$ and $\hat{\psi}_{i}^{2}$ are the projections of $\psi_{i+1}^{1}, \psi_{i}^{2}$, respectively, onto $N_{i+1}$. For notational convenience suppose $\left(v_{i+2}-v_{i+1}\right) \cdot n_{i+1}^{\prime}>0$ and $\left(v_{i+2}-v_{i+1}\right)$. $n_{i+1}>0$, which holds after possibly changing the signs of the vectors. Using that $\left(v_{i+2}-v_{i+1}\right) \cdot\left(n_{i+1}-n_{i+1}^{\prime}\right)=0$ and recalling that $d_{i+1}$ is small, we calculate by a Taylor expansion

$$
\begin{aligned}
\hat{\psi}_{i+1}^{1} & =\arccos \left(\frac{\left(v_{i+2}-v_{i+1}\right) \cdot n_{i+1}^{\prime}}{\left|v_{i+2}-v_{i+1}\right|\left|n_{i+1}^{\prime}\right|}\right) \\
& =\arccos \left(\frac{\left(v_{i+2}-v_{i+1}\right) \cdot n_{i+1}}{\left|v_{i+2}-v_{i+1}\right|\left(\left|n_{i+1}\right|+\mathrm{O}\left(d_{i+1}^{2}\right)\right)}\right) \\
& =\psi_{i+1}^{1}+\mathrm{O}\left(d_{i+1}^{2}\right),
\end{aligned}
$$

where $\mathrm{O}(\cdot)$ is universal. Likewise, we have $\hat{\psi}_{i}^{2}=\psi_{i}^{2}+\mathrm{O}\left(d_{i+1}^{2}\right)$. Since $\varphi_{i+1}=$ $\hat{\psi}_{i+1}^{1}+\hat{\psi}_{i}^{2}$, to conclude (28a), it therefore remains to show

$$
d_{i+1}^{2} \leqq c\left(\Delta\left(z_{\frac{i+1}{2}, j, 0}\right)+\Delta\left(z_{\frac{i+3}{2}, j, 0}\right)\right)
$$

for a universal constant $c>0$. To see this, we first note that we have $d_{i+1}=0$ whenever $\Delta\left(z_{\frac{i+1}{2}, j, 0}\right)+\Delta\left(z_{\frac{i+3}{2}, j, 0}\right)=0$. Indeed, if $\Delta\left(z_{\frac{i+1}{2}, j, 0}\right)+\Delta\left(z_{\frac{i+3}{2}, j, 0}\right)=0$, the high symmetry of the atoms in the cells with centers $z_{\frac{i+1}{2}, j, 0}$ and $\frac{z_{i+3}, j, 0}{2}$ (cf. (18)) implies that the three normal vectors $n_{i}, n_{i+1}$, and $n_{i+2}$ are coplanar. Thus, $x_{0}$ is contained in $N_{i+1}$ and therefore $d_{i+1}=0$.

Note that $d_{i+1}^{2}, \Delta\left(z_{\frac{i+1}{2}, j, 0}\right)$, and $\Delta\left(z_{\frac{i+3}{2}, j, 0}\right)$ are functions of the positions of the atoms contained in the adjacent cells with center $z_{\frac{i+1}{2}, j, 0}, \frac{i+3}{2}, j, 0$, denoted by $\tilde{\boldsymbol{y}}=\left(\tilde{y}_{1}, \ldots, \tilde{y}_{14}\right) \in \mathbb{R}^{3 \times 14}$. By (18) we find that $\Delta\left(z_{\frac{i+1}{2}, j, 0}\right)+\Delta\left(z_{\frac{i+3}{2}, j, 0}\right)=$ $\left(\tilde{\boldsymbol{y}}-\boldsymbol{y}^{0}\right)^{T} \mathcal{Q}\left(\tilde{\boldsymbol{y}}-\boldsymbol{y}^{0}\right)$ is quadratic with $\mathcal{Q} \in \mathbb{R}^{42 \times 42}$, where $\boldsymbol{y}^{0}$ denotes the atomic positions of $\mathcal{F}^{\prime} \in \mathscr{F}(\mu)$. Moreover, the fact that $d_{i+1}^{2}$ is smooth as a function in $\tilde{\boldsymbol{y}}$, a Taylor expansion, and $d_{i+1} \leqq \delta$ yield $d_{i+1}^{2} \leqq C\left|\tilde{\boldsymbol{y}}-\boldsymbol{y}^{0}\right|^{2}$ for a universal constant $C>0$. Now (29) follows from the property that $d_{i+1}=0$ whenever $\Delta\left(z_{\frac{i+1}{2}, j, 0}\right)+\Delta\left(z_{\frac{i+3}{2}, j, 0}\right)=0$.

The second estimate (28b) can be shown along similar lines. This concludes the proof.

\section{Properties of the Reduced Energy: Proof of Lemma 4.2, Proposition 4.3, and Proposition 3.4}

In this section we investigate the properties of the symmetric energy and the reduced energy as introduced in (23) and (24), respectively. 


\subsection{Proof of Lemma 4.2}

We start with the relation of the cell energy (13) and the symmetric energy (23).

Proof of Lemma 4.2. In the proof we let $\lambda=\lambda_{2}$. Given the cell energy, the symmetric energy, and the constraints (20)-(21), we observe that it suffices to show

$$
v_{2}\left(\lambda_{1}\right)+v_{2}\left(\lambda_{3}\right) \geqq 2 v_{2}\left(\tilde{\mu} / 2+2 \lambda \cos \alpha_{i}\right)-c_{0} \ell^{-4}\left(\gamma_{1}-\gamma_{2}\right)^{2} \text { for } i=1,2
$$

for a constant $c_{0}$ only depending on $v_{2}$ and $v_{3}$. First, with the notation of (20), particularly recalling $\lambda_{3}=\left|x_{8}-x_{2}\right|=\left|2\left(z_{i, j, k}^{\text {dual }}-x_{2}\right)\right|$, we see

$$
\lambda_{3}^{2}=\left(\tilde{\mu}-\lambda_{4}\right)^{2}+4\left|\left(x_{2}-z_{i, j, k}^{\mathrm{dual}}\right) \cdot e_{2}\right|^{2}+4\left|\left(x_{2}-z_{i, j, k}^{\mathrm{dual}}\right) \cdot e_{3}\right|^{2} .
$$

As in the special case $\gamma_{1}=\gamma_{2}$ the points $x_{1}, x_{2}, z_{i, j, k}^{\text {dual }}$ are contained in one line and thus the latter two terms vanish, we obtain by a Taylor expansion $\lambda_{3}=\tilde{\mu}-\lambda_{4}+$ $\mathrm{O}\left(\left(\gamma_{1}-\gamma_{2}\right)^{2}\right)$, which together with (22) gives

$$
\lambda_{1}+\lambda_{3}=\tilde{\mu}+2 \lambda \cos \alpha_{1}+\mathrm{O}\left(\left(\gamma_{1}-\gamma_{2}\right)^{2}\right) .
$$

By a similar argument, interchanging the roles of $\lambda_{1}$ and $\lambda_{3}$, we also get

$$
\lambda_{1}+\lambda_{3}=\tilde{\mu}+2 \lambda \cos \alpha_{2}+\mathrm{O}\left(\left(\gamma_{1}-\gamma_{2}\right)^{2}\right) .
$$

Recall that $\left|\lambda_{1}-1\right|+\left|\lambda_{3}-1\right| \leqq \ell^{-4}$ and $\left|\gamma_{1}-\gamma_{2}\right| \leqq \ell^{-2}$ by assumption. Then by the convexity of $v_{2}$ in a neighborhood of 1 and a Taylor expansion we derive

$$
\begin{aligned}
v_{2}\left(\lambda_{1}\right)+v_{2}\left(\lambda_{3}\right) \geqq & 2 v_{2}\left(\tilde{\mu} / 2+\lambda \cos \alpha_{i}+\mathrm{O}\left(\left(\gamma_{1}-\gamma_{2}\right)^{2}\right)\right) \\
\geqq & 2 v_{2}\left(\tilde{\mu} / 2+\lambda \cos \alpha_{i}\right)-C\left|v_{2}^{\prime}\left(\tilde{\mu} / 2+\lambda \cos \alpha_{i}\right)\right|\left(\gamma_{1}-\gamma_{2}\right)^{2} \\
& -C\left(\gamma_{1}-\gamma_{2}\right)^{4}
\end{aligned}
$$

for $i=1,2$. We recall that $\left|v_{2}^{\prime}\left(\tilde{\mu} / 2+\lambda \cos \alpha_{i}\right)\right|=\mathrm{O}\left(\ell^{-4}\right)$ since $\left|\lambda_{1}-1\right|+\mid \lambda_{3}-$ $1|+| \gamma_{1}-\left.\gamma_{2}\right|^{2} \leqq 2 \ell^{-4}$, and $v_{2}$ is smooth and attains its minimum in 1. Moreover, observe that by $\left|\gamma_{1}-\gamma_{2}\right| \leqq \ell^{-2}$ we get $\left|\gamma_{1}-\gamma_{2}\right|^{4} \leqq \ell^{-4}\left|\gamma_{1}-\gamma_{2}\right|^{2}$. This concludes the proof of (30).

\subsection{Convexity of the Reduced Energy}

Let us now concentrate on the symmetric energy $E_{\mu, \gamma_{1}, \gamma_{2}}^{\mathrm{sym}}$ introduced in (23). We recall the definition of the angle $\beta=\beta(\alpha, \gamma)=2 \arcsin \left(\sin \alpha \sin \frac{\gamma}{2}\right)$ in (21) and for later use we note that the function $\beta$ is smooth on $\left[\frac{1}{2} \pi, \frac{3}{4} \pi\right] \times\left[\frac{3}{4} \pi, \pi\right]$ and satisfies

$$
\begin{aligned}
\partial_{\alpha} \beta(2 \pi / 3, \pi) & =-2, \quad \partial_{\alpha \alpha}^{2} \beta(2 \pi / 3, \pi)=0, \quad \partial_{\gamma} \beta(2 \pi / 3, \pi)=0, \\
\partial_{\gamma \gamma}^{2} \beta(2 \pi / 3, \pi) & =-\sqrt{3} / 2, \quad \partial_{\alpha \gamma}^{2} \beta(2 \pi / 3, \pi)=0 .
\end{aligned}
$$


More precisely, a Taylor expansion also shows

$$
\lim _{\ell \rightarrow \infty} \ell \partial_{\gamma} \beta\left(2 \pi / 3, \gamma_{\ell}\right)=\frac{\sqrt{3}}{2} \pi, \quad \lim _{\ell \rightarrow \infty} \ell^{2} \partial_{\alpha \alpha}^{2} \beta\left(2 \pi / 3, \gamma_{\ell}\right)=-2 \sqrt{3} \pi^{2},
$$

where $\gamma_{\ell}$ was defined in (4). For the exact expressions of the derivatives of the function $\beta$ we refer the reader to [56, Section 4]. Recall the definition of $\alpha_{\ell}^{\text {us }}$ in Proposition 3.1.

Lemma 6.1. (Angles of unstretched nanotubes). There exist $0<c_{1}<c_{2}$ and $\ell_{0} \in \mathbb{N}$ only depending on $v_{3}$ such that for all $\ell \geqq \ell_{0}$

$$
\alpha_{\ell}^{\text {us }}, \beta\left(\alpha_{\ell}^{\text {us }}, \gamma_{\ell}\right) \in\left(2 \pi / 3-c_{2} \ell^{-2}, 2 \pi / 3-c_{1} \ell^{-2}\right) \text {. }
$$

Proof. By Proposition 3.1 and the fact that $\alpha \mapsto \beta\left(\alpha, \gamma_{\ell}\right)$ is decreasing, we obtain $\alpha_{\ell}^{\text {us }} \geqq \alpha_{\ell}^{\text {ch }}$ and $\beta\left(\alpha_{\ell}^{\text {us }}, \gamma_{\ell}\right) \leqq \alpha_{\ell}^{\text {us }} \leqq 2 \pi / 3$. By [55, (11)] we have $2 \pi / 3-\alpha_{\ell}^{\text {ch }}=$ $\mathrm{O}\left(\ell^{-2}\right)$. Moreover, in view of (4), (6) and a Taylor expansion, we find $\alpha_{\ell}^{\text {us }}-$ $\beta\left(\alpha_{\ell}^{\mathrm{us}}, \gamma_{\ell}\right) \geqq C \ell^{-2}$. Summarizing, we get

$$
2 \pi / 3-\alpha_{\ell}^{\mathrm{us}} \leqq C \ell^{-2}, \quad 2 \pi-2 \alpha_{\ell}^{\mathrm{us}}-\beta\left(\alpha_{\ell}^{\mathrm{us}}, \gamma_{\ell}\right) \geqq C \ell^{-2}
$$

for some universal $C>0$. As $2 v_{3}(\alpha)+v_{3}\left(\beta\left(\alpha, \gamma_{\ell}\right)\right)$ is minimized at $\alpha=\alpha_{\ell}^{\text {us }}$ (see Proposition 3.1), we get $2 v_{3}^{\prime}\left(\alpha_{\ell}^{\text {us }}\right)+v_{3}^{\prime}\left(\beta\left(\alpha_{\ell}^{\text {us }}, \gamma_{\ell}\right)\right) \partial_{\alpha} \beta\left(\alpha_{\ell}^{\text {us }}, \gamma_{\ell}\right)=0$. Using (31a) and a Taylor expansion of $v_{3}^{\prime}$ around $2 \pi / 3$, we deduce that for $\ell_{0}$ large enough and all $\ell \geqq \ell_{0}$

$$
\frac{2 \pi / 3-\alpha_{\ell}^{\mathrm{us}}}{2 \pi / 3-\beta\left(\alpha_{\ell}^{\mathrm{us}}, \gamma_{\ell}\right)} \in\left[C^{\prime}, 1\right]
$$

for a constant $0<C^{\prime}<1$ only depending on $v_{3}$. This together with (33) concludes the proof.

Recall the minimization problem (24) for the symmetric energy introduced in (23). We proceed with the identification of the minimizers of (24).

Proposition 6.2. (Existence and uniqueness of minimizers). There exists $\delta>0$ depending only on $v_{2}, v_{3}$ such that, for any fixed $\mu \in[3-\delta, 3+\delta]$ and $\gamma=\left(\gamma_{1}, \gamma_{2}\right) \in[\pi-\delta, \pi]^{2}$, the minimization problem (24) has a unique solution $\left(\lambda^{*}(\mu, \gamma), \alpha_{1}^{*}(\mu, \gamma), \alpha_{2}^{*}(\mu, \gamma)\right)$, which satisfies

$$
\nabla E_{\mu, \gamma_{1}, \gamma_{2}}^{\mathrm{sym}}\left(\lambda^{*}(\mu, \gamma), \alpha_{1}^{*}(\mu, \gamma), \alpha_{2}^{*}(\mu, \gamma)\right)=0,
$$

where $\nabla$ denotes the derivative with respect to $\left(\lambda, \alpha_{1}, \alpha_{2}\right)$.

Proof. We start the proof with a direct computation of the derivatives. Replace $E_{\mu, \gamma_{1}, \gamma_{2}}^{\mathrm{sym}}$ by $\tilde{E}$ for notational convenience. We obtain

$$
\begin{aligned}
\partial_{\lambda} \tilde{E}\left(\lambda, \alpha_{1}, \alpha_{2}\right)= & 2 v_{2}^{\prime}(\lambda)+\sum_{i=1,2}\left(\frac{1}{2} \cos \alpha_{i} v_{2}^{\prime}\left(\mu / 2+\lambda \cos \alpha_{i}\right)\right), \\
\partial_{\alpha_{i}} \tilde{E}\left(\lambda, \alpha_{1}, \alpha_{2}\right)= & -\frac{1}{2} \lambda \sin \alpha_{i} v_{2}^{\prime}\left(\mu / 2+\lambda \cos \alpha_{i}\right) \\
& +v_{3}^{\prime}\left(\beta\left(\alpha_{i}, \gamma_{i}\right)\right) \partial_{\alpha} \beta\left(\alpha_{i}, \gamma_{i}\right)+2 v_{3}^{\prime}\left(\alpha_{i}\right), \quad i=1,2 .
\end{aligned}
$$


Moreover, for $i=1,2$

$$
\begin{aligned}
\partial_{\lambda \lambda}^{2} \tilde{E}\left(\lambda, \alpha_{1}, \alpha_{2}\right)= & 2 v_{2}^{\prime \prime}(\lambda)+\sum_{j=1,2}\left(\frac{1}{2} \cos ^{2} \alpha_{j} v_{2}^{\prime \prime}\left(\mu / 2+\lambda \cos \alpha_{j}\right)\right) \\
\partial_{\alpha_{i} \alpha_{i}}^{2} \tilde{E}\left(\lambda, \alpha_{1}, \alpha_{2}\right)= & \frac{1}{2} \lambda^{2} \sin ^{2} \alpha_{i} v_{2}^{\prime \prime}\left(\mu / 2+\lambda \cos \alpha_{i}\right) \\
& -\frac{1}{2} \lambda \cos \alpha_{i} v_{2}^{\prime}\left(\mu / 2+\lambda \cos \alpha_{i}\right)+2 v_{3}^{\prime \prime}\left(\alpha_{i}\right) \\
& +v_{3}^{\prime \prime}\left(\beta\left(\alpha_{i}, \gamma_{i}\right)\right)\left(\partial_{\alpha} \beta\left(\alpha_{i}, \gamma_{i}\right)\right)^{2} \\
& +v_{3}^{\prime}\left(\beta\left(\alpha_{i}, \gamma_{i}\right)\right) \partial_{\alpha \alpha}^{2} \beta\left(\alpha_{i}, \gamma_{i}\right) \\
\partial_{\lambda \alpha_{i}}^{2} \tilde{E}\left(\lambda, \alpha_{1}, \alpha_{2}\right)= & -\frac{1}{2} \sin \alpha_{i} v_{2}^{\prime}\left(\mu / 2+\lambda \cos \alpha_{i}\right) \\
& -\frac{1}{2} \lambda \sin \alpha_{i} \cos \alpha_{i} v_{2}^{\prime \prime}\left(\mu / 2+\lambda \cos \alpha_{i}\right) \\
\partial_{\alpha_{1} \alpha_{2}}^{2} \tilde{E}\left(\lambda, \alpha_{1}, \alpha_{2}\right)= & 0
\end{aligned}
$$

For notational convenience we define $s_{\text {ref }}:=(1,2 \pi / 3,2 \pi / 3)$. Recall that $\partial_{\alpha} \beta(2 \pi / 3, \pi)=-2$ by $(31 \mathrm{a}), \beta(2 \pi / 3, \pi)=2 \pi / 3$ by $(21), v_{3}^{\prime}(2 \pi / 3)=0$, $\cos (2 \pi / 3)=-1 / 2, \sin (2 \pi / 3)=\sqrt{3} / 2$. At the planar reference configuration $\mu=3, \gamma_{1}=\gamma_{2}=\pi, \alpha_{1}=\alpha_{2}=2 \pi / 3, \lambda=1$ the derivative then reads after some computation

$$
\begin{aligned}
& \partial_{\lambda \lambda}^{2} E_{3, \pi, \pi}^{\mathrm{sym}}\left(s_{\mathrm{ref}}\right)=\frac{9}{4} v_{2}^{\prime \prime}(1), \quad \partial_{\alpha_{i} \alpha_{i}}^{2} E_{3, \pi, \pi}^{\mathrm{sym}}\left(s_{\mathrm{ref}}\right)=\frac{3}{8} v_{2}^{\prime \prime}(1)+6 v_{3}^{\prime \prime}(2 \pi / 3), \quad i=1,2, \\
& \partial_{\lambda \alpha_{i}}^{2} E_{3, \pi, \pi}^{\mathrm{sym}}\left(s_{\mathrm{ref}}\right)=\frac{\sqrt{3}}{8} v_{2}^{\prime \prime}(1), \quad i=1,2, \quad \partial_{\alpha_{1} \alpha_{2}}^{2} E_{3, \pi, \pi}^{\mathrm{sym}}\left(s_{\mathrm{ref}}\right)=0 .
\end{aligned}
$$

We shall check the positivity of the Hessian matrix in a neighborhood of the reference configuration. Since

$$
\begin{aligned}
\operatorname{det}\left(D_{\alpha_{1} \alpha_{2}}^{2} E_{3, \pi, \pi}^{\mathrm{sym}}\left(s_{\mathrm{ref}}\right)\right)= & \left(\partial_{\alpha_{1} \alpha_{1}}^{2} E_{3, \pi, \pi}^{\mathrm{sym}}\left(s_{\mathrm{ref}}\right)\right)^{2} \\
\operatorname{det}\left(D^{2} E_{3, \pi, \pi}^{\mathrm{sym}}\left(s_{\mathrm{ref}}\right)\right)= & \left(\partial_{\alpha_{1} \alpha_{1}}^{2} E_{3, \pi, \pi}^{\mathrm{sym}}\left(s_{\mathrm{ref}}\right)\right)^{2} \partial_{\lambda \lambda}^{2} E_{3, \pi, \pi}^{\mathrm{sym}}\left(s_{\mathrm{ref}}\right) \\
& -2\left(\partial_{\lambda \alpha_{1}}^{2} E_{3, \pi, \pi}^{\mathrm{sym}}\left(s_{\mathrm{ref}}\right)\right)^{2} \partial_{\alpha_{1} \alpha_{1}}^{2} E_{3, \pi, \pi}^{\mathrm{sym}}\left(s_{\mathrm{ref}}\right)
\end{aligned}
$$

are positive, the principal minors of the Hessian matrix $D^{2} E_{3, \pi, \pi}^{\mathrm{sym}}(1,2 \pi / 3,2 \pi / 3)$ are positive. Due to the smoothness of the potentials $v_{2}, v_{3}$ and the mapping $(\alpha, \gamma) \mapsto \beta(\alpha, \gamma)$, we get that for $\delta^{\prime}>0$ sufficiently small the principal minors of the Hessian matrix $D^{2} E_{\mu, \gamma_{1}, \gamma_{2}}^{\mathrm{sym}}\left(\lambda, \alpha_{1}, \alpha_{2}\right)$ are positive for all $\left(\lambda, \alpha_{1}, \alpha_{2}\right) \in D_{\delta^{\prime}}$ and for all $\mu \in\left[3-\delta^{\prime}, 3+\delta^{\prime}\right],\left(\gamma_{1}, \gamma_{2}\right) \in\left[\pi-\delta^{\prime}, \pi\right]^{2}$, where

$$
D_{\delta^{\prime}}:=\left[1-\delta^{\prime}, 1+\delta^{\prime}\right] \times\left[2 \pi / 3-\delta^{\prime}, 2 \pi / 3+\delta^{\prime}\right]^{2} .
$$

Since we have shown that $E_{\mu, \gamma_{1}, \gamma_{2}}^{\mathrm{sym}}$ is strictly convex on $D_{\delta^{\prime}}$, it follows that it has a unique minimizer $\left(\lambda^{*}(\mu, \gamma), \alpha_{1}^{*}(\mu, \gamma), \alpha_{2}^{*}(\mu, \gamma)\right)$ for all $\mu \in\left[3-\delta^{\prime}, 3+\delta^{\prime}\right]$ and 
$\gamma=\left(\gamma_{1}, \gamma_{2}\right) \in\left[\pi-\delta^{\prime}, \pi\right]^{2}$. Moreover, a continuity argument shows that

$$
\begin{aligned}
& \left(\lambda^{*}(\mu, \gamma), \alpha_{1}^{*}(\mu, \gamma), \alpha_{2}^{*}(\mu, \gamma)\right) \rightarrow\left(\lambda^{*}(3, \pi, \pi), \alpha_{1}^{*}(3, \pi, \pi), \alpha_{2}^{*}(3, \pi, \pi)\right) \\
& \quad=(1,2 \pi / 3,2 \pi / 3)
\end{aligned}
$$

as $\gamma \rightarrow(\pi, \pi)$ and $\mu \rightarrow 3$. Recalling (23) and the fact that $v_{2}$ and $v_{3}$ attain their minimum exactly at 1 and $2 \pi / 3$, respectively, we find $\inf _{\left(\lambda, \alpha_{1}, \alpha_{2}\right) \notin D_{\delta^{\prime}}} E_{\mu, \gamma_{1}, \gamma_{2}}^{\mathrm{syy}}\left(\lambda, \alpha_{1}\right.$, $\left.\alpha_{2}\right)>-3$. On the other hand, by (21), (23), and (36) we get $E_{\mu, \gamma_{1}, \gamma_{2}}^{\mathrm{sym}}\left(\lambda^{*}(\mu, \gamma)\right.$, $\left.\alpha_{1}^{*}(\mu, \gamma), \alpha_{2}^{*}(\mu, \gamma)\right) \rightarrow-3$ as $\gamma \rightarrow(\pi, \pi)$ and $\mu \rightarrow 3$. This shows that for all $\mu \in\left[3-\delta^{\prime \prime}, 3+\delta^{\prime \prime}\right]$ and $\gamma \in\left[\pi-\delta^{\prime \prime}, \pi\right]^{2}$, for some small $\delta^{\prime \prime}>0$, the triple $\left(\lambda^{*}(\mu, \gamma), \alpha_{1}^{*}(\mu, \gamma), \alpha_{2}^{*}(\mu, \gamma)\right)$ is the unique solution of the minimization problem (24). Moreover, if $\delta^{\prime \prime}>0$ is chosen small enough, the triple lies in the interior of $D_{\delta^{\prime}}$ and the first order optimality conditions (34) follow. We conclude the proof by setting $\delta=\min \left\{\delta^{\prime}, \delta^{\prime \prime}\right\}$.

We now study convexity properties of the reduced energy $E_{\text {red }}$ defined in (24). Recall the definition of $\gamma_{\ell}$ in (4) and the definition of $\mu_{\ell}^{\text {us }}$ in (9).

Proposition 6.3. (Convexity of reduced energy). There exists $\ell_{0} \in \mathbb{N}$ and for each $\ell \geqq \ell_{0}$ there exits $\varepsilon=\varepsilon(\ell)>0$ such that $E_{\text {red }}$ is strictly convex on $D_{\varepsilon}^{\ell}:=$ $\left[\mu_{\ell}^{\mathrm{us}}-\varepsilon, \mu_{\ell}^{\mathrm{us}}+\varepsilon\right] \times\left[\gamma_{\ell}-\varepsilon, \gamma_{\ell}+\varepsilon\right]^{2}$. Moreover, there exists $c_{0}^{\prime}>0$ depending only on $v_{2}$ and $v_{3}$ such that for all $\ell \geqq \ell_{0}$ and $\left(\mu, \gamma_{1}, \gamma_{2}\right) \in D_{\varepsilon}^{\ell}$

$E_{\text {red }}\left(\mu, \gamma_{1}, \gamma_{2}\right)=E_{\text {red }}\left(\mu, \gamma_{2}, \gamma_{1}\right) \geqq E_{\text {red }}\left(\mu, \frac{\gamma_{1}+\gamma_{2}}{2}, \frac{\gamma_{1}+\gamma_{2}}{2}\right)+c_{0}^{\prime} \ell^{-2}\left(\gamma_{1}-\gamma_{2}\right)^{2}$.

Proof. Choosing $\ell$ sufficiently large and $\varepsilon>0$ small we can suppose that $D_{\varepsilon}^{\ell} \subset$ $[3-\delta, 3+\delta] \times[\pi-\delta, \pi]^{2}$ with $\delta$ from Proposition 6.2 since $\mu_{\ell}^{\text {us }}=2-2 \cos \alpha_{\ell}^{\text {us }} \rightarrow 3$ as $\ell \rightarrow \infty$. Then (34) holds for $\left(\mu, \gamma_{1}, \gamma_{2}\right) \in D_{\varepsilon}^{\ell}$.

We drop the brackets $\left(\mu, \gamma_{1}, \gamma_{2}\right)$ and indicate the unique solution at $\left(\mu, \gamma_{1}, \gamma_{2}\right)$ by $\left(\lambda^{*}, \alpha_{1}^{*}, \alpha_{2}^{*}\right)$ for notational convenience. Taking the partial derivatives and making use of the first order optimality conditions (34), we get

$$
\begin{aligned}
\partial_{\mu} E_{\mathrm{red}}\left(\mu, \gamma_{1}, \gamma_{2}\right)= & \frac{d}{d \mu} E_{\mu, \gamma_{1}, \gamma_{2}}^{\mathrm{sym}}\left(\lambda^{*}, \alpha_{1}^{*}, \alpha_{2}^{*}\right) \\
= & \frac{\partial E_{\mu, \gamma_{1}, \gamma_{2}}^{\mathrm{sym}}}{\partial \mu}\left(\lambda^{*}, \alpha_{1}^{*}, \alpha_{2}^{*}\right) \\
& +\nabla E_{\mu, \gamma_{1}, \gamma_{2}}^{\mathrm{sym}}\left(\lambda^{*}, \alpha_{1}^{*}, \alpha_{2}^{*}\right) \cdot\left(\partial_{\mu} \lambda^{*}, \partial_{\mu} \alpha_{1}^{*}, \partial_{\mu} \alpha_{2}^{*}\right) \\
= & \frac{\partial E_{\mu, \gamma_{1}, \gamma_{2}}^{\mathrm{sym}}}{\partial \mu}\left(\lambda^{*}, \alpha_{1}^{*}, \alpha_{2}^{*}\right)=\sum_{j=1,2} \frac{1}{4} v_{2}^{\prime}\left(\mu / 2+\lambda^{*} \cos \alpha_{j}^{*}\right),
\end{aligned}
$$

where $\nabla$ denotes the derivative with respect to $\left(\lambda, \alpha_{1}, \alpha_{2}\right)$. Likewise, we get for $i=1,2$

$$
\partial_{\gamma_{i}} E_{\mathrm{red}}\left(\mu, \gamma_{1}, \gamma_{2}\right)=\frac{\partial E_{\mu, \gamma_{1}, \gamma_{2}}^{\mathrm{sym}}}{\partial \gamma_{i}}\left(\lambda^{*}, \alpha_{1}^{*}, \alpha_{2}^{*}\right)=v_{3}^{\prime}\left(\beta\left(\alpha_{i}^{*}, \gamma_{i}\right)\right) \partial_{\gamma} \beta\left(\alpha_{i}^{*}, \gamma_{i}\right) \text {. }
$$


Next we compute the second derivatives and obtain

$$
\begin{aligned}
\partial_{\mu \mu}^{2} E_{\mathrm{red}}\left(\mu, \gamma_{1}, \gamma_{2}\right)= & \sum_{j=1,2} \frac{1}{4} v_{2}^{\prime \prime}\left(\mu / 2+\lambda^{*} \cos \alpha_{j}^{*}\right) w_{j, \mu}\left(\mu, \gamma_{1}, \gamma_{2}\right) \\
\partial_{\gamma_{i} \gamma_{i}}^{2} E_{\mathrm{red}}\left(\mu, \gamma_{1}, \gamma_{2}\right)= & v_{3}^{\prime}\left(\beta\left(\alpha_{i}^{*}, \gamma_{i}\right)\right)\left(\partial_{\gamma \gamma}^{2} \beta\left(\alpha_{i}^{*}, \gamma_{i}\right)+\partial_{\gamma \alpha}^{2} \beta\left(\alpha_{i}^{*}, \gamma_{i}\right) \partial_{\gamma_{i}} \alpha_{i}^{*}\right) \\
& +v_{3}^{\prime \prime}\left(\beta\left(\alpha_{i}^{*}, \gamma_{i}\right)\right) \partial_{\gamma} \beta\left(\alpha_{i}^{*}, \gamma_{i}\right) \cdot\left(\partial_{\gamma} \beta\left(\alpha_{i}^{*}, \gamma_{i}\right)\right. \\
& \left.+\partial_{\alpha} \beta\left(\alpha_{i}^{*}, \gamma_{i}\right) \partial_{\gamma_{i}} \alpha_{i}^{*}\right), i=1,2 \\
\partial_{\mu \gamma_{i}}^{2} E_{\mathrm{red}}\left(\mu, \gamma_{1}, \gamma_{2}\right)= & \sum_{j=1,2} \frac{1}{4} v_{2}^{\prime \prime}\left(\mu / 2+\lambda^{*} \cos \alpha_{j}^{*}\right) w_{j, \gamma_{i}}\left(\mu, \gamma_{1}, \gamma_{2}\right), \quad i=1,2, \\
\partial_{\gamma_{1} \gamma_{2}}^{2} E_{\mathrm{red}}\left(\mu, \gamma_{1}, \gamma_{2}\right)= & v_{3}^{\prime}\left(\beta\left(\alpha_{1}^{*}, \gamma_{1}\right)\right) \partial_{\gamma \alpha}^{2} \beta\left(\alpha_{1}^{*}, \gamma_{1}\right) \partial_{\gamma_{2}} \alpha_{1}^{*} \\
& +v_{3}^{\prime \prime}\left(\beta\left(\alpha_{1}^{*}, \gamma_{1}\right)\right) \partial_{\gamma} \beta\left(\alpha_{1}^{*}, \gamma_{1}\right) \partial_{\alpha} \beta\left(\alpha_{1}^{*}, \gamma_{1}\right) \partial_{\gamma_{2}} \alpha_{1}^{*}
\end{aligned}
$$

where for brevity we have introduced

$$
\begin{aligned}
& w_{j, \mu}\left(\mu, \gamma_{1}, \gamma_{2}\right)=1 / 2+\partial_{\mu} \lambda^{*} \cos \alpha_{j}^{*}-\lambda^{*} \sin \alpha_{j}^{*} \partial_{\mu} \alpha_{j}^{*}, \quad j=1,2, \\
& w_{j, \gamma_{i}}\left(\mu, \gamma_{1}, \gamma_{2}\right)=\partial_{\gamma_{i}} \lambda^{*} \cos \alpha_{j}^{*}-\lambda^{*} \sin \alpha_{j}^{*} \partial_{\gamma_{i}} \alpha_{j}^{*}, \quad i, j=1,2 .
\end{aligned}
$$

We now exploit the identity $\nabla E_{\mu, \gamma_{1}, \gamma_{2}}^{\mathrm{sym}}\left(\lambda^{*}, \alpha_{1}^{*}, \alpha_{2}^{*}\right)=0$ : differentiating (35) with respect to $\mu, \gamma_{1}$ or $\gamma_{2}$, respectively, we obtain

$$
\begin{aligned}
0= & 2 v_{2}^{\prime \prime}\left(\lambda^{*}\right) \partial_{X} \lambda^{*}+\sum_{j=1,2}\left(-\frac{1}{2} \sin \alpha_{j}^{*} \partial_{X} \alpha_{j}^{*} v_{2}^{\prime}\left(\mu / 2+\lambda^{*} \cos \alpha_{j}^{*}\right)\right) \\
& +\sum_{j=1,2}\left(\frac{1}{2} \cos \alpha_{j}^{*} v_{2}^{\prime \prime}\left(\mu / 2+\lambda^{*} \cos \alpha_{j}^{*}\right) w_{j, X}\left(\mu, \gamma_{1}, \gamma_{2}\right)\right), \\
0= & -\frac{1}{2} v_{2}^{\prime}\left(\mu / 2+\lambda^{*} \cos \alpha_{j}^{*}\right)\left(\partial_{X} \lambda^{*} \sin \alpha_{j}^{*}+\lambda^{*} \cos \alpha_{j}^{*} \partial_{X} \alpha_{j}^{*}\right) \\
& -\frac{1}{2} \lambda^{*} \sin \alpha_{j}^{*} v_{2}^{\prime \prime}\left(\mu / 2+\lambda^{*} \cos \alpha_{j}^{*}\right) w_{j, X}\left(\mu, \gamma_{1}, \gamma_{2}\right) \\
& +v_{3}^{\prime}\left(\beta\left(\alpha_{j}^{*}, \gamma_{j}\right)\right) \partial_{\alpha \alpha}^{2} \beta\left(\alpha_{j}^{*}, \gamma_{j}\right) \partial_{X} \alpha_{j}^{*} \\
& +v_{3}^{\prime \prime}\left(\beta\left(\alpha_{j}^{*}, \gamma_{j}\right)\right)\left(\partial_{\alpha} \beta\left(\alpha_{j}^{*}, \gamma_{j}\right)\right)^{2} \partial_{X} \alpha_{j}^{*} \\
& +2 v_{3}^{\prime \prime}\left(\alpha_{j}^{*}\right) \partial_{X} \alpha_{j}^{*}+z_{j, X}\left(\mu, \gamma_{1}, \gamma_{2}\right), \quad j=1,2,
\end{aligned}
$$

where $X \in\left\{\mu, \gamma_{1}, \gamma_{2}\right\}$ and where we have defined for brevity

$$
\begin{aligned}
z_{j, \gamma_{j}}\left(\mu, \gamma_{1}, \gamma_{2}\right)= & v_{3}^{\prime}\left(\beta\left(\alpha_{j}^{*}, \gamma_{j}\right)\right) \partial_{\alpha \gamma} \beta\left(\alpha_{j}^{*}, \gamma_{j}\right) \\
& +v_{3}^{\prime \prime}\left(\beta\left(\alpha_{j}^{*}, \gamma_{j}\right)\right) \partial_{\alpha} \beta\left(\alpha_{j}^{*}, \gamma_{j}\right) \partial_{\gamma} \beta\left(\alpha_{j}^{*}, \gamma_{j}\right), \\
z_{j, \gamma_{i}}\left(\mu, \gamma_{1}, \gamma_{2}\right)= & z_{j, \mu}\left(\mu, \gamma_{1}, \gamma_{2}\right)=0, \quad i \neq j .
\end{aligned}
$$

For brevity let $t_{\mathrm{ref}}^{\ell}:=\left(\mu_{\ell}^{\text {us }}, \gamma_{\ell}, \gamma_{\ell}\right)$ and $t_{\mathrm{ref}}:=(3, \pi, \pi)$. Observe that $t_{\mathrm{ref}}^{\ell} \rightarrow t_{\mathrm{ref}}$ as $\ell \rightarrow \infty$ by (4), (9), and Lemma 6.1. Moreover, by (36) we get that the unique 
solution of the problem (24) corresponding to $t_{\text {ref }}^{\ell}$ converges to $(1,2 \pi / 3,2 \pi / 3)$, in particular $\alpha_{j}^{*}\left(t_{\mathrm{ref}}^{\ell}\right) \rightarrow 2 \pi / 3$ for $j=1,2$. We also recall $\beta\left(\alpha_{j}^{*}\left(t_{\mathrm{ref}}^{\ell}\right), \gamma_{\ell}\right) \rightarrow 2 \pi / 3$ for $j=1,2$ (see (21)). Using $v_{2}^{\prime}(1)=v_{3}^{\prime}(2 \pi / 3)=0, \cos (2 \pi / 3)=-1 / 2$, $\sin (2 \pi / 3)=\sqrt{3} / 2$ and (31) we then deduce from (45)-(46)

$$
\begin{aligned}
& 0=2 v_{2}^{\prime \prime}(1) \partial_{X} \lambda^{*}\left(t_{\mathrm{ref}}\right)-\frac{1}{4} v_{2}^{\prime \prime}(1) \sum_{j=1,2} w_{j, X}\left(t_{\mathrm{ref}}\right), \\
& 0=-v_{2}^{\prime \prime}(1) w_{j, X}\left(t_{\mathrm{ref}}\right)+8 \sqrt{3} v_{3}^{\prime \prime}(2 \pi / 3) \partial_{X} \alpha_{j}^{*}\left(t_{\mathrm{ref}}\right), \quad j=1,2,
\end{aligned}
$$

as $\ell \rightarrow \infty$, where $X \in\left\{\mu, \gamma_{1}, \gamma_{2}\right\}$. Inserting the identities into (44), we obtain, after some elementary but tedious calculations,

$$
\begin{aligned}
& w_{1, \mu}\left(t_{\mathrm{ref}}\right)=w_{2, \mu}\left(t_{\mathrm{ref}}\right)=4 / K, \quad w_{1, \gamma_{i}}\left(t_{\mathrm{ref}}\right)=w_{2, \gamma_{i}}\left(t_{\mathrm{ref}}\right)=0, \quad i=1,2, \\
& \partial_{\mu} \lambda^{*}\left(t_{\mathrm{ref}}\right)=1 / K, \quad \partial_{\mu} \alpha_{1}^{*}\left(t_{\mathrm{ref}}\right)=\partial_{\mu} \alpha_{2}^{*}\left(t_{\mathrm{ref}}\right)=v_{2}^{\prime \prime}(1) /\left(2 \sqrt{3} K v_{3}^{\prime \prime}(2 \pi / 3)\right),
\end{aligned}
$$

where $K:=9+v_{2}^{\prime \prime}(1) /\left(2 v_{3}^{\prime \prime}(2 \pi / 3)\right)$. In particular, the last two equalities of the first line together with (47) yield that $\partial_{\gamma_{i}} \lambda^{*}, \partial_{\gamma_{i}} \alpha_{1}^{*}$, and $\partial_{\gamma_{i}} \alpha_{2}^{*}$ vanish at $t_{\text {ref. Thus, }}$ by a Taylor expansion in terms of $1 / \ell$ the limits $w_{j, \gamma_{i}}^{\infty}:=\lim _{\ell \rightarrow \infty} \ell w_{j, \gamma_{i}}\left(t_{\mathrm{ref}}^{\ell}\right)$, $\partial_{\gamma_{i}} \lambda^{\infty}:=\lim _{\ell \rightarrow \infty} \ell \partial_{\gamma_{i}} \lambda^{*}\left(t_{\mathrm{ref}}^{\ell}\right)$, and $\partial_{\gamma_{i}} \alpha_{j}^{\infty}:=\lim _{\ell \rightarrow \infty} \ell \partial_{\gamma_{i}} \alpha_{j}^{*}\left(t_{\mathrm{ref}}^{\ell}\right)$ for $i, j=1,2$ exist and are finite.

By Lemma 6.1 and the fact that $v_{3}$ is smooth with minimum at $2 \pi / 3$ we note that one has $\left|v_{3}^{\prime}\left(\beta\left(\alpha_{\ell}^{\text {us }}, \gamma_{\ell}\right)\right)\right| \leqq C \ell^{-2}$ for a constant only depending on $v_{3}$. Consequently, multiplying the estimates in (45)-(46) by $\ell$ and letting $\ell \rightarrow \infty$ we get using (31) and (32)

$$
\begin{aligned}
& 0=2 v_{2}^{\prime \prime}(1) \partial_{\gamma_{i}} \lambda^{\infty}-\frac{1}{4} v_{2}^{\prime \prime}(1) \sum_{j=1,2} w_{j, \gamma_{i}}^{\infty}, \quad i=1,2, \\
& 0=-\frac{1}{4} v_{2}^{\prime \prime}(1) w_{j, \gamma_{i}}^{\infty}+2 \sqrt{3} v_{3}^{\prime \prime}(2 \pi / 3) \partial_{\gamma_{i}} \alpha_{j}^{\infty}-v_{3}^{\prime \prime}(2 \pi / 3) \pi \delta_{i j}, \quad i, j=1,2,
\end{aligned}
$$

where $\delta_{i j}$ denotes the Kronecker delta. As before, inserting the identities into (44b), we obtain after some tedious calculations

$$
\begin{aligned}
\sum_{j=1,2} w_{j, \gamma_{i}}^{\infty} & =-\frac{2 \pi}{K}, \quad \sum_{j=1,2} \partial_{\gamma_{i}} \alpha_{j}^{\infty}=\frac{\pi}{2 \sqrt{3}}-\frac{\pi v_{2}^{\prime \prime}(1)}{4 \sqrt{3} K v_{3}^{\prime \prime}(2 \pi / 3)}, \\
\partial_{\gamma_{i}} \alpha_{i}^{\infty} & =\frac{\pi}{2 \sqrt{3}}-\frac{\pi v_{2}^{\prime \prime}(1)}{4 \sqrt{3} K v_{3}^{\prime \prime}(2 \pi / 3)}-\frac{\pi}{K K^{\infty}}, \quad \partial_{\gamma_{i}} \alpha_{j}^{\infty}=\frac{\pi}{K K^{\infty}}, \quad i \neq j,
\end{aligned}
$$

for $i=1,2$ with $K$ as defined after (48) and $K^{\infty}:=64 \sqrt{3} v_{3}^{\prime \prime}(2 \pi / 3) / v_{2}^{\prime \prime}(1)+4 \sqrt{3}$. Moreover, we notice by (31b) and Lemma 6.1 that

$$
v_{3}^{\prime}\left(\beta\left(\alpha_{\ell}^{\mathrm{us}}, \gamma_{\ell}\right)\right) \partial_{\gamma \gamma}^{2} \beta\left(\alpha_{\ell}^{\mathrm{us}}, \gamma_{\ell}\right) \geqq 0
$$


for $\ell$ sufficiently large. With this at hand, we go back to (40)-(43) and derive as $\ell \rightarrow \infty$ by (31), (32), (48), and (49)

$$
\begin{aligned}
\partial_{\mu \mu}^{2} E_{\mathrm{red}}\left(t_{\mathrm{ref}}^{\ell}\right)= & \frac{2 v_{2}^{\prime \prime}(1)}{K}+\mathrm{O}\left(\ell^{-1}\right) \\
\partial_{\gamma_{i} \gamma_{i}}^{2} E_{\mathrm{red}}\left(t_{\mathrm{ref}}^{\ell}\right) \geqq & \ell^{-2}\left(v_{3}^{\prime \prime}(2 \pi / 3) \frac{3}{4} \pi^{2}-v_{3}^{\prime \prime}(2 \pi / 3) \sqrt{3} \pi \partial_{\gamma_{i}} \alpha_{i}^{\infty}\right)+\mathrm{O}\left(\ell^{-3}\right) \\
= & \ell^{-2} v_{3}^{\prime \prime}(2 \pi / 3) \pi^{2}\left(\frac{1}{4}+\frac{v_{2}^{\prime \prime}(1)}{4 K v_{3}^{\prime \prime}(2 \pi / 3)}+\frac{\sqrt{3}}{K K^{\infty}}\right) \\
& +\mathrm{O}\left(\ell^{-3}\right), i=1,2 \\
\partial_{\mu \gamma_{i}}^{2} E_{\mathrm{red}}\left(t_{\mathrm{ref}}^{\ell}\right)= & -\ell^{-1} \frac{\pi v_{2}^{\prime \prime}(1)}{2 K}+\mathrm{O}\left(\ell^{-2}\right), i=1,2 \\
\partial_{\gamma_{1} \gamma_{2}}^{2} E_{\mathrm{red}}\left(t_{\mathrm{ref}}^{\ell}\right)= & -\ell^{-2} v_{3}^{\prime \prime}(2 \pi / 3) \sqrt{3} \pi \partial_{\gamma_{1}} \alpha_{2}^{\infty}+\mathrm{O}\left(\ell^{-3}\right) \\
= & -\ell^{-2} v_{3}^{\prime \prime}(2 \pi / 3) \frac{\sqrt{3} \pi^{2}}{K K^{\infty}}+\mathrm{O}\left(\ell^{-3}\right)
\end{aligned}
$$

We now check the positivity of the Hessian $D^{2} E_{\text {red }}$ by considering the minors $H_{1}=\partial_{\gamma_{2} \gamma_{2}}^{2} E_{\text {red }}, H_{2}=\operatorname{det}\left(D_{\gamma_{1} \gamma_{2}}^{2} E_{\text {red }}\right)$ and $H_{3}=\operatorname{det}\left(D^{2} E_{\text {red }}\right)$. First, we get for $\ell \in \mathbb{N}$ sufficiently large

$$
H_{1}\left(t_{\mathrm{ref}}^{\ell}\right) \geqq \ell^{-2} v_{3}^{\prime \prime}(2 \pi / 3) \frac{\pi^{2}}{4}>0, \quad H_{2}\left(t_{\mathrm{ref}}^{\ell}\right) \geqq \ell^{-4}\left(v_{3}(2 \pi / 3)^{\prime \prime}\right)^{2} \pi^{4}(1 / 4)^{2}>0
$$

and finally for $\ell$ large enough

$$
\begin{aligned}
H_{3}\left(t_{\mathrm{ref}}^{\ell}\right)= & \left(\partial_{\gamma_{2} \gamma_{2}}^{2} E_{\mathrm{red}}-\partial_{\gamma_{1} \gamma_{2}}^{2} E_{\mathrm{red}}\right) \cdot\left(\partial _ { \mu \mu } ^ { 2 } E _ { \mathrm { red } } \left(\partial_{\gamma_{2} \gamma_{2}}^{2} E_{\mathrm{red}}\right.\right. \\
& \left.\left.+\partial_{\gamma_{1} \gamma_{2}}^{2} E_{\mathrm{red}}\right)-2\left(\partial_{\mu \gamma_{1}}^{2} E_{\mathrm{red}}\right)^{2}\right) \\
\geqq & \ell^{-4} v_{3}^{\prime \prime}(2 \pi / 3) \frac{\pi^{2}}{4}\left(\frac{\pi^{2} v_{2}^{\prime \prime}(1) v_{3}^{\prime \prime}(2 \pi / 3)}{2 K}\right. \\
& \left.+\frac{\pi^{2}\left(v_{2}^{\prime \prime}(1)\right)^{2}}{2 K^{2}}-2 \frac{\pi^{2}\left(v_{2}^{\prime \prime}(1)\right)^{2}}{4 K^{2}}\right)>0 .
\end{aligned}
$$

Due to the smoothness of the potentials $v_{2}, v_{3}$, the mapping $(\alpha, \gamma) \mapsto \beta(\alpha, \gamma)$, and the solutions $\left(\lambda^{*}, \alpha_{1}^{*}, \alpha_{2}^{*}\right)$ as functions of $\left(\mu, \gamma_{1}, \gamma_{2}\right)$, we get that for $\ell_{0} \in \mathbb{N}$ sufficiently large and $\varepsilon>0$ small (depending on $\ell$ ) $H_{i}\left(\mu, \gamma_{1}, \gamma_{2}\right)>0$ for $i=$ $1,2,3$ for all $\left(\mu, \gamma_{1}, \gamma_{2}\right) \in\left[\mu_{\ell}^{\text {us }}-\varepsilon, \mu_{\ell}^{\text {us }}+\varepsilon\right] \times\left[\gamma_{\ell}-\varepsilon, \gamma_{\ell}+\varepsilon\right]^{2}$.

It remains to confirm (37). The first identity is a consequence of the fact that $E_{\mu, \gamma_{1}, \gamma_{2}}^{\mathrm{sym}}$ is symmetric in $\left(\alpha_{1}, \gamma_{1}\right)$ and $\left(\alpha_{2}, \gamma_{2}\right)$. Recalling (50) and the fact that $D^{2} E_{\text {red }}$ is positive definite, we can control the eigenvalues of $\ell^{2} D^{2} E_{\text {red }}$ from below and find $\ell^{2} D^{2} E_{\text {red }} \geqq 8 c_{0}^{\prime} \mathbf{I}+\mathrm{O}\left(\ell^{-1}\right)$ for some constant $c_{0}^{\prime}$ depending only on $v_{2}^{\prime \prime}(1)$ and $v_{3}^{\prime \prime}(2 \pi / 3)$, where $\mathbf{I}$ denotes the identity matrix. This implies the second estimate of (37). 


\subsection{Proof of Proposition 4.3 and Proposition 3.4}

We are now in the position to show the main properties of $E_{\text {red }}$.

Proof of Proposition 4.3. Property 2 follows directly from Proposition 6.3 if the intervals $M^{\ell}, G^{\ell}$ are chosen appropriately depending on $\varepsilon$, with $\varepsilon$ from Proposition 6.3.

In Proposition 6.2 we have seen that for given $\left(\mu, \gamma_{1}, \gamma_{2}\right) \in M^{\ell} \times G^{\ell} \times G^{\ell}$ there is a unique solution $\left(\lambda^{*}, \alpha_{1}^{*}, \alpha_{2}^{*}\right)$ of the minimization problem (24). In particular, if $\gamma_{1}=\gamma_{2}$ we obtain $\alpha^{*}:=\alpha_{1}^{*}=\alpha_{2}^{*}$ as then (24) is completely symmetric in $\alpha_{1}$ and $\alpha_{2}$. This proves Property 1 .

We now specifically consider the case $\gamma_{1}=\gamma_{2}=\gamma_{\ell}$ and denote the minimizer in (24) by $\left(\lambda^{\mu}, \alpha^{\mu}, \alpha^{\mu}\right)$. We observe that $\lambda_{1}^{\mu}:=\mu / 2+\lambda^{\mu} \cos \alpha^{\mu}, \lambda_{2}^{\mu}:=\lambda^{\mu}$, and $\sigma^{\mu}:=-\lambda^{\mu} \cos \alpha^{\mu}$ satisfy the relations (3) and (5). Then by (8), (23), and the fact that $n=4 m \ell$ we derive

$$
\begin{aligned}
E_{\text {red }}\left(\mu, \gamma_{\ell}, \gamma_{\ell}\right) & =2 v_{2}\left(\lambda^{\mu}\right)+v_{2}\left(\mu / 2+\lambda^{\mu} \cos \alpha^{\mu}\right)+4 v_{3}\left(\alpha^{\mu}\right)+2 v_{3}\left(\beta\left(\alpha^{\mu}, \gamma_{\ell}\right)\right) \\
& =2 v_{2}\left(\lambda_{2}^{\mu}\right)+v_{2}\left(\lambda_{1}^{\mu}\right)+4 v_{3}\left(\alpha^{\mu}\right)+2 v_{3}\left(\beta\left(\alpha^{\mu}, \gamma_{\ell}\right)\right) \\
& =\frac{1}{2 m \ell} E\left(\mathcal{F}_{\lambda_{1}^{\mu}, \lambda_{2}^{\mu}, \mu}\right),
\end{aligned}
$$

which concludes the proof of Property 5.

To see Property 3, we introduce $g(\gamma)=E_{\text {red }}(\mu, \gamma, \gamma)$ for $\mu \in M^{\ell}$. By (39) we have

$$
g^{\prime}(\gamma)=\sum_{i=1,2} \partial_{\gamma_{i}} E_{\mathrm{red}}(\mu, \gamma, \gamma)=2 v_{3}^{\prime}\left(\beta\left(\alpha^{*}, \gamma\right)\right) \partial_{\gamma} \beta\left(\alpha^{*}, \gamma\right)
$$

where $\alpha^{*}=\alpha^{*}(\mu, \gamma, \gamma)$. Using (32) and the fact that $v_{3}^{\prime}\left(\beta\left(\alpha^{*}, \gamma\right)\right)<0$ since $\beta\left(\alpha^{*}, \gamma\right)<2 \pi / 3$, we get $g^{\prime}(\gamma)<0$. Moreover, taking again (32) and Lemma 6.1 into account, a Taylor expansion shows $\left|g^{\prime}(\gamma)\right| \leqq C \ell^{-3}$ for some $C>0$ only depending on $v_{3}$. This shows Property 3 .

Finally, we show Property 4 . The strict convexity of $\mu \mapsto E_{\text {red }}\left(\mu, \gamma_{\ell}, \gamma_{\ell}\right)$ follows from (50) and a continuity argument, exactly as in the proof of Proposition 6.3. To show that the mapping is strictly increasing for $\mu>\mu_{\ell}^{\text {us }}$, we have to show that for $\mu>\mu_{\ell}^{\mathrm{us}}$

$$
\mu / 2+\lambda^{\mu} \cos \alpha^{\mu}>1
$$

as then the property follows from (38). Using the monotonicity properties of $v_{2}$ we see that the first-order optimality conditions (34) and (35a) imply

$$
\mu / 2+\lambda^{\mu} \cos \alpha^{\mu}>1 \Leftrightarrow \lambda^{\mu}>1 \text {. }
$$

We prove (51) by contradiction. Suppose $\lambda^{\mu} \leqq 1$. This together with the fact $\mu>\mu_{\ell}^{\text {us }}=2-2 \cos \alpha_{\ell}^{\text {us }}$ (see (9)) and $\cos \alpha^{\mu}<0$ would imply by (52)

$$
2 \cos \alpha^{\mu}-2 \cos \alpha_{\ell}^{\text {us }}+1=\mu_{\ell}^{\text {us }}-1+2 \cos \alpha^{\mu}<\mu-1+2 \lambda^{\mu} \cos \alpha^{\mu} \leqq 1
$$


and thus $\alpha^{\mu}>\alpha_{\ell}^{\text {us }}$. By the optimality condition in the unstretched case (see (35b) and recall that bond lengths are all equal to 1) we get

$$
v_{3}^{\prime}\left(\beta\left(\alpha_{\ell}^{\mathrm{us}}, \gamma_{\ell}\right)\right) \partial_{\alpha} \beta\left(\alpha_{\ell}^{\mathrm{us}}, \gamma_{\ell}\right)+2 v_{3}^{\prime}\left(\alpha_{\ell}^{\mathrm{us}}\right)=0 \text {. }
$$

Consider the mapping $\alpha \mapsto v_{3}^{\prime}\left(\beta\left(\alpha, \gamma_{\ell}\right)\right) \partial_{\alpha} \beta\left(\alpha, \gamma_{\ell}\right)+2 v_{3}^{\prime}(\alpha)$ and observe that its derivative is

$$
v_{3}^{\prime}\left(\beta\left(\alpha, \gamma_{\ell}\right)\right) \partial_{\alpha \alpha}^{2} \beta\left(\alpha, \gamma_{\ell}\right)+v_{3}^{\prime \prime}\left(\beta\left(\alpha, \gamma_{\ell}\right)\right)\left(\partial_{\alpha} \beta\left(\alpha, \gamma_{\ell}\right)\right)^{2}+2 v_{3}^{\prime \prime}(\alpha)
$$

Thus, the mapping is strictly increasing in a left neighborhood of $2 \pi / 3$ by (32) and the fact that $\beta\left(\alpha, \gamma_{\ell}\right)<2 \pi / 3$. Since $\alpha^{\mu}>\alpha_{\ell}^{\text {us }}$, this gives

$$
v_{3}^{\prime}\left(\beta\left(\alpha^{\mu}, \gamma_{\ell}\right)\right) \partial_{\alpha} \beta\left(\alpha^{\mu}, \gamma_{\ell}\right)+2 v_{3}^{\prime}\left(\alpha^{\mu}\right)>0 \text {. }
$$

In view of (35b) and the first order optimality conditions (34), we get $\mu / 2+$ $\lambda^{\mu} \cos \alpha^{\mu}>1$, which contradicts the last inequality in (53). Consequently, (51) holds, which concludes the proof.

We close this section with the proof of Proposition 3.4.

Proof of Proposition 3.4. Let $M^{\ell}$ be the interval given by Proposition 4.3. The strict convexity of the mapping $\mu \mapsto E_{\min }(\mu)$ on $M^{\ell}$ as well as $\frac{d^{2}}{d \mu^{2}} E_{\min }\left(\mu_{\ell}^{\text {us }}\right) \geqq c 2 m \ell \geqq$ $c n$ follow from Properties 4 and 5 of Proposition 4.3. The fact that the energy minimum is attained at $\mu_{\ell}^{\mathrm{us}}$ follows from the definition of $\mu_{\ell}^{\mathrm{us}}$, see Proposition 3.1 and (9). This shows Property 1.

Now consider Property 2. We define $\lambda_{1}^{\mu}=\mu / 2+\lambda^{\mu} \cos \alpha^{\mu}, \lambda_{2}^{\mu}=\lambda^{\mu}$ with $\lambda^{\mu}, \alpha^{\mu}$ being the solution of (24) for $\mu$ and $\gamma_{1}=\gamma_{2}=\gamma_{\ell}$ (cf. Proposition 4.3(v)) and use (48b) to obtain $\partial_{\mu} \lambda_{2}^{\mu}\left(t_{\text {ref }}\right)=\partial_{\mu} \lambda^{*}\left(t_{\text {ref }}\right)=1 / K$ and $\partial_{\mu} \lambda_{1}^{\mu}\left(t_{\text {ref }}\right)=1 / 2-$ $\partial_{\mu} \lambda^{*}\left(t_{\mathrm{ref}}\right) / 2-\sqrt{3} \partial_{\mu} \alpha_{1}^{*}\left(t_{\mathrm{ref}}\right) / 2=4 / K$ with $K=9+v_{2}^{\prime \prime}(1) /\left(2 v_{3}^{\prime \prime}(2 \pi / 3)\right)$. (Recall the definition $t_{\text {ref }}=(3, \pi, \pi)$.) Consequently, by a standard continuity argument we see that $\lambda_{1}^{\mu}$ and $\lambda_{2}^{\mu}$ increase continuously for $\mu \in M^{\ell}$, possibly passing to a smaller (not relabeled) open interval $M^{\ell}$ containing $\mu_{\ell}^{\text {us }}$. The proof of the fact that $\mu>\mu_{\ell}^{\mathrm{us}}$ implies $\lambda_{1}^{\mu}, \lambda_{2}^{\mu}>1$ is already contained in the proof of Proposition 4.3, see particularly (51) and (52). The fact that $\mu<\mu_{\ell}^{\mathrm{us}}$ implies $\lambda_{1}^{\mu}, \lambda_{2}^{\mu}<1$ can be proved along similar lines.

To see Property 3, recall that by Proposition 3.1 we have $\alpha_{\ell}^{\text {us }}=\alpha^{\mu_{\ell}^{\text {us }}} \in$ $\left(\alpha_{\ell}^{\mathrm{ch}}, \alpha^{\mathrm{ru}}\right)$ in the unstretched case. By a continuity argument we particularly obtain the convergence of minimizers, i.e., $\alpha^{\mu} \rightarrow \alpha^{\mu_{\ell}^{\text {us }}}$ as $\mu \rightarrow \mu_{\ell}^{\text {us }}$. Consequently, again possibly passing to a smaller interval $M^{\ell}$, Property 3 follows. We finally concern ourselves with Property 4. Recall by (5) that the radius of the nanotube is given by

$$
\rho^{\mu}=\lambda_{2}^{\mu} \sin \alpha^{\mu} /(2 \sin (\pi /(2 \ell))) .
$$

We compute the derivative and obtain

$$
\partial_{\mu} \rho^{\mu}=\left(\lambda_{2}^{\mu} \cos \alpha^{\mu} \partial_{\mu} \alpha^{\mu}+\partial_{\mu} \lambda_{2}^{\mu} \sin \alpha^{\mu}\right) /(2 \sin (\pi /(2 \ell))) .
$$


By (48b) the derivative at the unstrechted planar reference configuration is

$$
\begin{aligned}
\lim _{\ell \rightarrow \infty} \partial_{\mu} \rho^{\mu_{\ell}^{\mathrm{us}} \cdot(2 \sin (\pi /(2 \ell)))} & =-\frac{1}{2} \partial_{\mu} \alpha_{1}^{*}\left(t_{\mathrm{ref}}\right)+\frac{1}{2} \sqrt{3} \partial_{\mu} \lambda^{*}\left(t_{\mathrm{ref}}\right) \\
& =\frac{\sqrt{3}}{2 K}\left(1-\frac{v_{2}^{\prime \prime}(1)}{6 v_{3}^{\prime \prime}(2 \pi / 3)}\right) .
\end{aligned}
$$

Consequently, whenever $\left.v_{2}^{\prime \prime}(1) \neq 6 v_{3}^{\prime \prime}(2 \pi / 3)\right)$, by a continuity argument the sign of $\partial_{\mu} \rho^{\mu}$ for $\ell \in \mathbb{N}$ large in a small neighborhood of $\mu_{\ell}^{\text {us }}$ only depends on the sign of $v_{2}^{\prime \prime}(1)-6 v_{3}^{\prime \prime}(2 \pi / 3)$.

\section{Energy Defect Controls Symmetry Defect: Proof of Theorem 4.4}

This section is devoted to the proof of Theorem 4.4. The fact that the minimum of the cell energy is attained for a special configuration with high symmetry (see (20)) essentially relies on convexity properties of the cell energy $E_{\text {cell }}$ defined in (13). Throughout the section we consider a cell consisting of eight points $\boldsymbol{x}=$ $\left(x_{1}, \ldots, x_{8}\right) \in \mathbb{R}^{3 \times 8}$ as defined before (13), see Fig. 5. Likewise, the bond lengths are again denoted by $b_{1}, \ldots, b_{8}$ and the angles by $\varphi_{1}, \ldots, \varphi_{10}$, see Fig. 6 . With a slight abuse of notation we denote the cell energy for a given configuration $\boldsymbol{x}$ by $E_{\text {cell }}(\boldsymbol{x})$.

\subsection{Relation Between Atomic Positions, Bonds, and Angles}

We will investigate the convexity properties of $E_{\text {cell }}$ near the planar reference configuration $\boldsymbol{x}^{0}=\left(x_{1}^{0}, \ldots, x_{8}^{0}\right) \in \mathbb{R}^{3 \times 8}$ defined by

$$
\begin{aligned}
& x_{1}^{0}=(-1,0,0), \quad x_{2}^{0}=(1,0,0), \quad x_{3}^{0}=(-1 / 2, \sqrt{3} / 2,0), \\
& x_{4}^{0}=(1 / 2, \sqrt{3} / 2,0), \quad x_{5}^{0}=(1 / 2,-\sqrt{3} / 2,0), \quad x_{6}^{0}=(-1 / 2,-\sqrt{3} / 2,0), \\
& x_{7}^{0}=(-2,0,0), \quad x_{8}^{0}=(2,0,0) .
\end{aligned}
$$

Moreover, we introduce the unstretched kink configuration $\boldsymbol{x}_{\mathrm{kink}}^{\ell}=\left(x_{1}^{\mathrm{kink}}, \ldots\right.$, $\left.x_{8}^{\text {kink }}\right) \in \mathbb{R}^{3 \times 8}$ by

$$
\begin{aligned}
& x_{1}^{\text {kink }}=\left(-1 / 2-\sigma^{\mathrm{us}}, 0,0\right), \\
& x_{2}^{\text {kink }}=\left(1 / 2+\sigma^{\mathrm{us}}, 0,0\right), \\
& x_{3}^{\text {kink }}=\left(-1 / 2, \sin \alpha_{\ell}^{\mathrm{us}} \sin \left(\gamma_{\ell} / 2\right), \sin \alpha_{\ell}^{\mathrm{us}} \cos \left(\gamma_{\ell} / 2\right)\right), \\
& x_{4}^{\mathrm{kink}}=\left(1 / 2, \sin \alpha_{\ell}^{\mathrm{us}} \sin \left(\gamma_{\ell} / 2\right), \sin \alpha_{\ell}^{\mathrm{us}} \cos \left(\gamma_{\ell} / 2\right)\right), \\
& x_{5}^{\mathrm{kink}}=\left(1 / 2,-\sin \alpha_{\ell}^{\mathrm{us}} \sin \left(\gamma_{\ell} / 2\right), \sin \alpha_{\ell}^{\mathrm{us}} \cos \left(\gamma_{\ell} / 2\right)\right), \\
& x_{6}^{\mathrm{kink}}=\left(-1 / 2,-\sin \alpha_{\ell}^{\mathrm{us}} \sin \left(\gamma_{\ell} / 2\right), \sin \alpha_{\ell}^{\mathrm{us}} \cos \left(\gamma_{\ell} / 2\right)\right), \\
& x_{7}^{\text {kink }}=\left(-3 / 2-\sigma^{\mathrm{us}}, 0,0\right), \\
& x_{8}^{\text {kink }}=\left(3 / 2+\sigma^{\mathrm{us}}, 0,0\right),
\end{aligned}
$$


where $\gamma_{\ell}=\pi(1-1 / \ell)$ and $\sigma^{\mathrm{us}}=-\cos \alpha_{\ell}^{\mathrm{us}}$ with $\alpha_{\ell}^{\mathrm{us}}$ as given by Proposition 3.1 (cf. also (5)). Note that $\boldsymbol{x}_{\text {kink }}^{\ell}$ represents the mutual position of atoms in a cell for

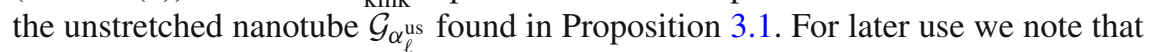
by Lemma 6.1 and a Taylor expansion we find

$$
\left|x^{0}-x_{\text {kink }}^{\ell}\right| \leqq C \ell^{-1}
$$

for some universal $C>0$ large enough. In order to discuss the convexity properties of $E_{\text {cell }}$ we need to introduce a specific basis of $\mathbb{R}^{3 \times 8}$, i.e., the space of cell configurations. This will consist of three collections of vectors, denoted by $\mathcal{V}_{\text {degen }}, \mathcal{V}_{\text {good }}$, and $\mathcal{V}_{\text {bad }}$, where the sets are defined as follows: We introduce the translations and infinitesimal rotations

$$
\begin{aligned}
\mathcal{V}_{\text {trans }} & =\left\{\left(e_{1}, \ldots, e_{1}\right),\left(e_{2}, \ldots, e_{2}\right),\left(e_{3}, \ldots, e_{3}\right)\right\} \subset \mathbb{R}^{3 \times 8} \\
\mathcal{V}_{\text {rot }} & =\left\{\boldsymbol{v}_{1}:=\left(\begin{array}{ccc}
0 & 1 & 0 \\
-1 & 0 & 0 \\
0 & 0 & 0
\end{array}\right) \boldsymbol{x}^{0}, \boldsymbol{v}_{2}:=\left(\begin{array}{ccc}
0 & 0 & 1 \\
0 & 0 & 0 \\
-1 & 0 & 0
\end{array}\right) \boldsymbol{x}^{0}, \boldsymbol{v}_{3}:=\left(\begin{array}{ccc}
0 & 0 & 0 \\
0 & 0 & 1 \\
0 & -1 & 0
\end{array}\right) \boldsymbol{x}^{0}\right\} \\
& \subset \mathbb{R}^{3 \times 8}
\end{aligned}
$$

and set $\mathcal{V}_{\text {degen }}=\mathcal{V}_{\text {trans }} \cup \mathcal{V}_{\text {rot. }}$ The family $\mathcal{V}_{\text {good }}$ contains the 13 vectors

$$
\begin{aligned}
\boldsymbol{u}_{1}= & (-1,0,0|1,0,0|-1 / 2, \sqrt{3} / 2,0|1 / 2, \sqrt{3} / 2,0| 1 / 2,-\sqrt{3} / 2,0 \mid-1 / 2, \\
& -\sqrt{3} / 2,0|0,0,0| 0,0,0), \\
\boldsymbol{u}_{2}= & (0,0,0|0,0,0| 1 / 2, \sqrt{3} / 2,0|-1 / 2, \sqrt{3} / 2,0| 0,0,0|0,0,0| 0,0,0 \mid 0,0,0), \\
\boldsymbol{u}_{3}= & (0,0,0|1,0,0| 0,0,0|1,0,0| 1,0,0|0,0,0| 0,0,0 \mid 0,0,0), \\
\boldsymbol{u}_{4}= & (0,0,0|1 / 2,-\sqrt{3} / 2,0| 1 / 2, \sqrt{3} / 2,0|-1 / 2, \sqrt{3} / 2,0| 1,0,0|0,0,0| 0,0,0 \mid 0,0,0), \\
\boldsymbol{u}_{5}= & (0,0,0|0,0,0| 0,0,0|0,0,0| 0,0,0|0,0,0|-1,0,0 \mid 0,0,0), \\
\boldsymbol{u}_{6}= & (0,0,0|0,0,0| 0,0,0|0,0,0| 0,0,0|0,0,0|-1,0,0 \mid 1,0,0), \\
\boldsymbol{u}_{7}= & (\sqrt{3}, 0,0|0,0,0| 0,1,0|0,0,0| 0,0,0|0,-1,0| 0,0,0 \mid 0,0,0), \\
\boldsymbol{u}_{8}= & (0,0,0|0,0,0| \sqrt{3} / 2,-1 / 2,0|\sqrt{3} / 2,1 / 2,0| 0,0,0|0,0,0| 0,0,0 \mid 0,0,0), \\
\boldsymbol{u}_{9}= & (\sqrt{3} / 2,1 / 2,0|-\sqrt{3} / 2,1 / 2,0| 0,1,0|0,1,0| 0,0,0|0,0,0| 0,0,0 \mid 0,0,0), \\
\boldsymbol{u}_{10}= & (0,0,0|0,0,0| 0,0,0|0,0,0| 0,0,0|0,0,0| 0,1,0 \mid 0,0,0), \\
\boldsymbol{u}_{11}= & (0,0,0|0,0,0| 0,0,0|0,0,0| 0,0,0|0,0,0| 0,1,0 \mid 0,1,0), \\
\boldsymbol{u}_{12}= & (1,0,0|0,0,0| 0,0,0|0,0,0| 0,0,0|0,0,0| 0,0,0 \mid 0,0,0), \\
\boldsymbol{u}_{13}= & (0,1,0|0,0,0| 0,0,0|0,0,0| 0,0,0|0,0,0| 0,0,0 \mid 0,0,0)
\end{aligned}
$$

The first 6 vectors keep the angles fixed and modify only the bond lengths, see Fig. 9. The vectors $\boldsymbol{u}_{8}, \ldots, \boldsymbol{u}_{11}$ keep the bond lengths fixed to first order and change the angles, see Fig. 10. Eventually, the remaining vectors $\boldsymbol{u}_{12}$ and $\boldsymbol{u}_{13}$ modify both angles and bonds as in Fig. 11.

By $\mathcal{V}_{\text {bad }}$ we denote the collection of the vectors

$$
\begin{aligned}
& (0,0,1|0,0,0| 0,0,0|0,0,0| 0,0,0|0,0,0| 0,0,0 \mid 0,0,0), \\
& (0,0,1|0,0,0| 0,0,1|0,0,0| 0,0,0|0,0,0| 0,0,0 \mid 0,0,0),
\end{aligned}
$$



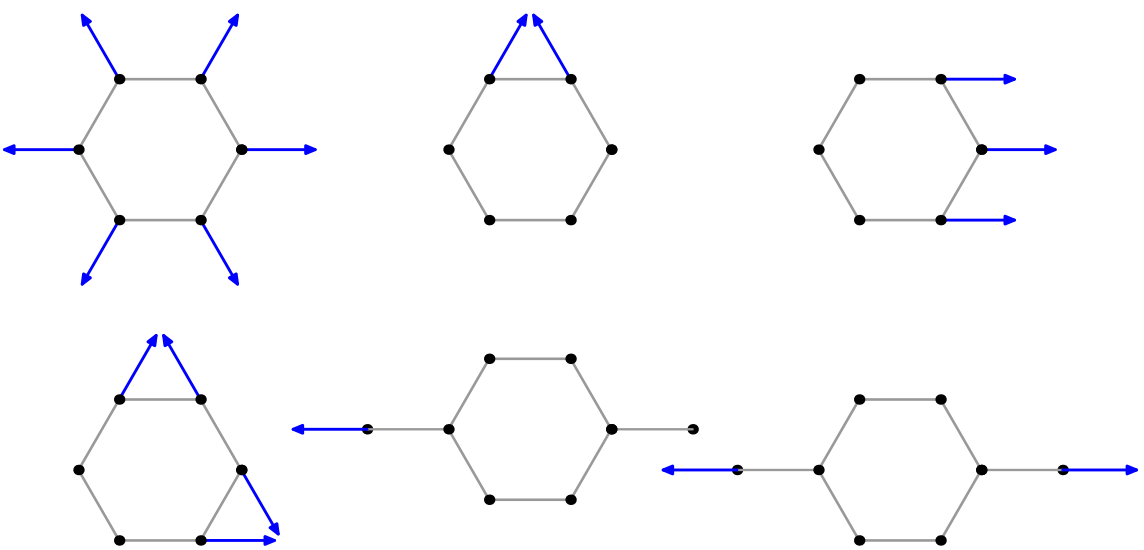

Fig. 9. Vectors $\boldsymbol{u}_{1}, \ldots, \boldsymbol{u}_{6}$ in $\mathcal{V}_{\text {good }}$ keep the angles fixed (ordered from left to right both in the first and in the second line)
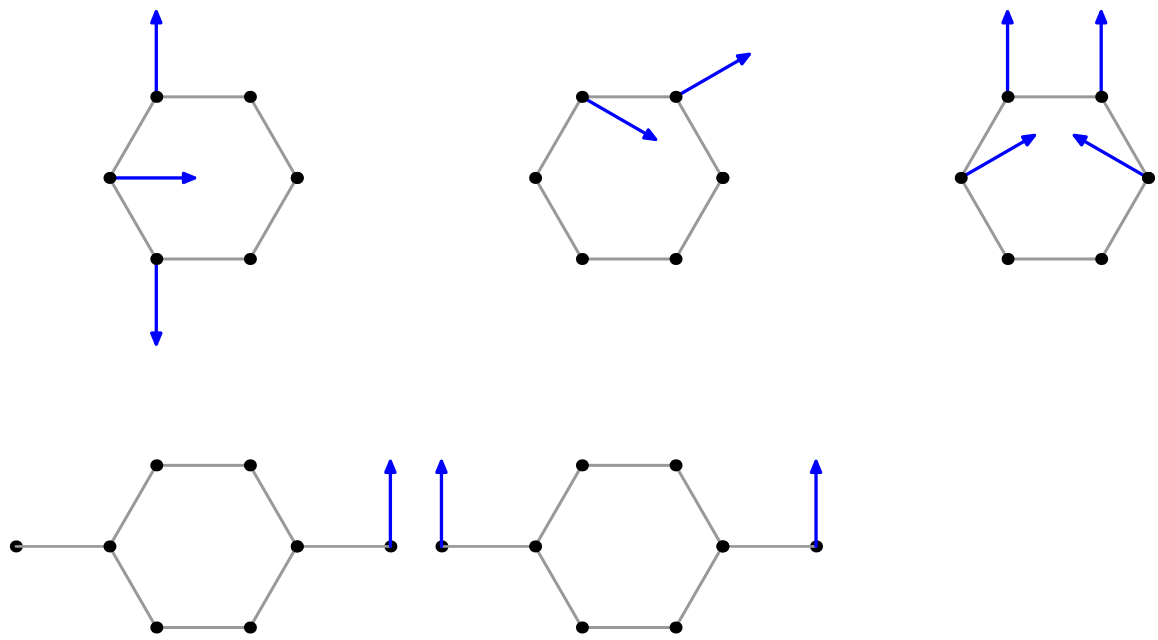

Fig. 10. Vectors $\boldsymbol{u}_{7}, \ldots, \boldsymbol{u}_{11}$ in $\mathcal{V}_{\text {good }}$ keep the bond lengths fixed (ordered from left to right both in the first and in the second line)
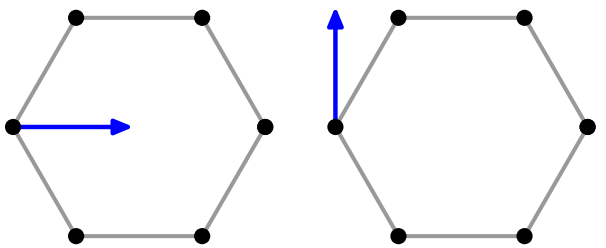

Fig. 11. Vectors $\boldsymbol{u}_{12}$ and $\boldsymbol{u}_{13}$ in $\mathcal{V}_{\text {good }}$ keep neither angles nor bond lengths fixed (ordered from left to right) 


$$
\begin{aligned}
& (0,0,1|0,0,0| 0,0,0|0,0,1| 0,0,1|0,0,0| 0,0,0 \mid 0,0,0), \\
& (0,0,0|0,0,0| 0,0,0|0,0,0| 0,0,0|0,0,0| 0,0,1 \mid 0,0,0), \\
& (0,0,0|0,0,0| 0,0,0|0,0,0| 0,0,0|0,0,0| 0,0,1 \mid 0,0,1) .
\end{aligned}
$$

It is elementary to check that the vectors $\mathcal{V}_{\text {degen }} \cup \mathcal{V}_{\text {good }} \cup \mathcal{V}_{\text {bad }}$ are linearly independent and thus form a basis of $\mathbb{R}^{3 \times 8}$. Note that the vectors in $\mathcal{V}_{\text {good }}$ are perpendicular to the vectors in $\mathcal{V}_{\text {bad }}$.

Clearly, the cell energy is strictly convex as a function of the bond lengths and angles by the assumptions on the potentials $v_{2}$ and $v_{3}$. Our goal is to show that the same property holds if the cell energy is given as a function of the atomic positions. To this end, we introduce the mapping $T=\left(T^{a}, T^{b}\right): \mathbb{R}^{3 \times 8} \rightarrow \mathbb{R}^{18}$ defined by

$$
T_{i}^{a}(\boldsymbol{x})=\varphi_{i} \text { for } i=1, \ldots, 10, \quad T_{i}^{b}(\boldsymbol{x})=b_{i} \text { for } i=1, \ldots, 8 .
$$

Then the cell energy reads as

$$
E_{\mathrm{cell}}(\boldsymbol{x})=\sum_{i=1}^{8} \kappa_{i}^{b} v_{2}\left(T_{i}^{b}(\boldsymbol{x})\right)+\sum_{i=1}^{10} \kappa_{i}^{a} v_{3}\left(T_{i}^{a}(\boldsymbol{x})\right)
$$

with the factors $\kappa_{1}^{b}=\kappa_{2}^{b}=\kappa_{7}^{b}=\kappa_{8}^{b}=1 / 4, \kappa_{3}^{b}=\kappa_{4}^{b}=\kappa_{5}^{b}=\kappa_{6}^{b}=1 / 2$, $\kappa_{1}^{a}=\kappa_{2}^{a}=1, \kappa_{3}^{a}=\ldots=\kappa_{10}^{a}=1 / 2$.

Before analyzing the mapping $T$, we need to introduce some more notation for the sum of angles $\varphi_{i}$. From here on, we denote by $\boldsymbol{e}_{1}, \ldots, \boldsymbol{e}_{10}$ the canonical basis of $\mathbb{R}^{10}$, and we let

$$
a_{1}:=e_{1}+\ldots+e_{6}, \quad a_{2}:=e_{1}+e_{7}+e_{8}, \quad a_{3}:=e_{2}+e_{9}+e_{10}
$$

be vectors in $\mathbb{R}^{10}$. Elementary geometry yields $T^{a}\left(\boldsymbol{x}^{0}\right) \cdot \boldsymbol{a}_{1}=4 \pi$ and $T^{a}\left(\boldsymbol{x}^{0}\right) \cdot \boldsymbol{a}_{j}=$ $2 \pi$ for $j=2,3$ as well as $T^{a}(\boldsymbol{x}) \cdot \boldsymbol{a}_{1} \leqq 4 \pi$ and $T^{a}(\boldsymbol{x}) \cdot \boldsymbol{a}_{j} \leqq 2 \pi$ for $j=2,3$ for each $x \in \mathbb{R}^{3 \times 8}$. Indeed, the sum of the interior angles in a hexagon is always smaller or equal to $4 \pi$ and exactly $4 \pi$ if the hexagon is planar. Likewise one argues for a triple junction.

Lemma 7.1. (Properties of $T$ ). The mapping $T$ is smooth in a neighborhood of $\boldsymbol{x}^{0}$. There is a constant $c_{\mathrm{kink}}>0$ such that:

1. $\operatorname{Ker}\left(D T\left(\boldsymbol{x}^{0}\right)\right)=\operatorname{span}\left(\mathcal{V}_{\text {degen }} \cup \mathcal{V}_{\text {bad }}\right), \quad \operatorname{dim}\left(\operatorname{Ker}\left(D T\left(\boldsymbol{x}^{0}\right)\right)\right)=11$,

2. $\operatorname{dim}\left(\operatorname{Ker}\left(D T^{a}\left(\boldsymbol{x}^{0}\right)\right)\right)=17$,

3. $\left(\boldsymbol{v}^{T} D^{2} T^{a}\left(\boldsymbol{x}^{0}\right) \boldsymbol{v}\right) \cdot \boldsymbol{a}_{j} \leqq 0$ for $j=1,2,3$, for all $\boldsymbol{v} \in \mathbb{R}^{3 \times 8}$,

4. $\sum_{j=1}^{3}\left(\boldsymbol{v}^{T} D^{2} T^{a}\left(\boldsymbol{x}^{0}\right) \boldsymbol{v}\right) \cdot \boldsymbol{a}_{j} \leqq-c_{\text {kink }}\left|\boldsymbol{v}-\boldsymbol{v}_{\mathrm{degen}}\right|^{2}$ for all $v \in \operatorname{span}\left(\mathcal{V}_{\text {degen }} \cup \mathcal{V}_{\text {bad }}\right)$, where $\boldsymbol{v}_{\text {degen }}$ is the orthogonal projection of $\boldsymbol{v}$ onto $\operatorname{span}\left(\mathcal{V}_{\mathrm{degen}}\right)$. 
Proof. First, to see Property 1, we note that $\operatorname{span}\left(\mathcal{V}_{\text {degen }} \cup \mathcal{V}_{\text {bad }}\right)$ is a subset of $\operatorname{Ker}\left(D T\left(x^{0}\right)\right)$ since each vector in $\mathcal{V}_{\text {degen }} \cup \mathcal{V}_{\text {bad }}$ does not change bond lengths and angles to first order. On the other hand, each vector in $\mathcal{V}_{\text {good }}$ changes bond lengths or angles to first order and is therefore not contained in the kernel of $D T\left(x^{0}\right)$. Indeed, the first six vectors of $\mathcal{V}_{\text {good }}$ are directions of perturbations that do not change angles to first order, but bond lengths. Vectors $\boldsymbol{u}_{7}, \ldots, \boldsymbol{u}_{11}$ are perturbations that do not change bond lengths in first order, but angles. Vectors $\boldsymbol{u}_{12}$ and $\boldsymbol{u}_{13}$ are in-plane displacements of a single atom and change both bond lengths and angles to first order. More precisely, for the changes of bond lengths we get

$$
\begin{array}{ll}
D T^{b}\left(\boldsymbol{x}^{0}\right) \boldsymbol{u}_{1} \|(1,1,1,1,1,1,-1,-1), & D T^{b}\left(\boldsymbol{x}^{0}\right) \boldsymbol{u}_{2} \|(0,-1,1,1,0,0,0,0), \\
D T^{b}\left(\boldsymbol{x}^{0}\right) \boldsymbol{u}_{3} \|(1,1,0,0,0,0,0,-1), & D T^{b}\left(\boldsymbol{x}^{0}\right) \boldsymbol{u}_{4} \|(2,-2,2,4,-2,0,0,-1), \\
D T^{b}\left(\boldsymbol{x}^{0}\right) \boldsymbol{u}_{5} \|(0,0,0,0,0,0,1,0), & D T^{b}\left(\boldsymbol{x}^{0}\right) \boldsymbol{u}_{6} \|(0,0,0,0,0,0,1,1), \\
D T^{b}\left(\boldsymbol{x}^{0}\right) \boldsymbol{u}_{12} \|(0,0,-1,0,0,-1,2,0), & D T^{b}\left(\boldsymbol{x}^{0}\right) \boldsymbol{u}_{13} \|(0,0,-1,0,0,1,0,0),
\end{array}
$$

where $\boldsymbol{w}_{1} \| \boldsymbol{w}_{2}$ indicates that $\boldsymbol{w}_{1}$ and $\boldsymbol{w}_{2}$ are linearly dependent. Likewise, for the changes of angles we have

$$
\begin{array}{ll}
D T^{a}\left(\boldsymbol{x}^{0}\right) \boldsymbol{u}_{7} \|(4,0,-3,1,1,-3,-2,-2,0,0), & D T^{a}\left(\boldsymbol{x}^{0}\right) \boldsymbol{u}_{8} \|(-1,1,2,-2,0,0,1,0,-1,0), \\
D T^{a}\left(\boldsymbol{x}^{0}\right) \boldsymbol{u}_{9} \|(-2,-2,1,1,1,1,1,1,1,1), & D T^{a}\left(\boldsymbol{x}^{0}\right) \boldsymbol{u}_{10} \|(0,0,0,0,0,0,0,0,-1,1), \\
D T^{a}\left(\boldsymbol{x}^{0}\right) \boldsymbol{u}_{11} \|(0,0,0,0,0,0,-1,1,-1,1), & D T^{a}\left(\boldsymbol{x}^{0}\right) \boldsymbol{u}_{12} \|(2,0,-1,0,0,-1,-1,-1,0,0), \\
D T^{a}\left(\boldsymbol{x}^{0}\right) \boldsymbol{u}_{13} \|(0,0,0,0,0,0,1,-1,0,0) . &
\end{array}
$$

(We prefer not to give details of the computation, but rather refer the reader to Figs. 9, 10 and 11 where the situation of the different directions is indicated.). It is elementary to check that the vectors $D T\left(\boldsymbol{x}^{0}\right) \boldsymbol{u}_{i}, i=1, \ldots, 13$, are linearly independent which concludes the proof of Property 1 by dimension counting.

Since $\operatorname{dim}\left(\operatorname{Ker}\left(D T\left(\boldsymbol{x}^{0}\right)\right)\right)=11$ and in $\mathcal{V}_{\text {good }}$ only the first six vectors do not change angles to first order, Property 2 holds.

Property 3 follows from the fact that the mapping $t \mapsto T^{a}\left(\boldsymbol{x}^{0}+t \boldsymbol{v}\right) \cdot \boldsymbol{a}_{j}$ has a local maximum at $t=0$ for $j=1,2,3$ and for all $v \in \mathbb{R}^{3 \times 8}$ as noticed before the statement of the lemma.

To see Property 4, we first consider the special case $\boldsymbol{v} \in \mathcal{V}_{\text {bad }}$. In this situation the property follows from an elementary computation, which we detail only in the case $\boldsymbol{v}=\left(e_{3}|0| \ldots \mid 0\right)$. In this case, after some calculations, we obtain $\left(T^{a}\left(\boldsymbol{x}^{0}+t \boldsymbol{v}\right)\right)_{i}=$ $\arccos \left(-1 / 2+3 t^{2} / 2\right)+\mathrm{O}\left(t^{3}\right) \leqq 2 \pi / 3-c t^{2}$ for some $c>0$ for $i=1,7$, 8, i.e., for the angles at the triple junction at point $x_{1}$. Using also Property 1 , this indeed implies $\left(\boldsymbol{v}^{T} D^{2} T^{a}\left(\boldsymbol{x}^{0}\right) \boldsymbol{v}\right) \cdot \boldsymbol{a}_{2} \leqq-c$, i.e., by a perturbation out of the plane the sum of the angles is reduced to second order. For the other triple junction and the interior angles of the hexagon we argue analogously. This shows the property for perturbations in the directions $\mathcal{V}_{\text {bad }}$. Likewise, we proceed for directions in $\operatorname{span}\left(\mathcal{V}_{\text {bad }}\right)$.

Now consider the general case $\boldsymbol{v}=\boldsymbol{v}_{\text {trans }}+\boldsymbol{v}_{\text {rot }}+\boldsymbol{v}_{\text {bad }} \in \operatorname{span}\left(\mathcal{V}_{\text {degen }} \cup \mathcal{V}_{\text {bad }}\right)$ for $\boldsymbol{v}_{\text {trans }} \in \operatorname{span}\left(\mathcal{V}_{\text {trans }}\right), \boldsymbol{v}_{\text {rot }} \in \operatorname{span}\left(\mathcal{V}_{\text {rot }}\right)$, and $\boldsymbol{v}_{\text {bad }} \in \operatorname{span}\left(\mathcal{V}_{\text {bad }}\right)$.

First, since $T(\boldsymbol{x}+\boldsymbol{w})=T(\boldsymbol{x})$ for all $\boldsymbol{x} \in \mathbb{R}^{3 \times 8}$ and all $\boldsymbol{w} \in \mathcal{V}_{\text {trans, }}$, we get $D T(\boldsymbol{x}) \boldsymbol{w}=0$ and $\boldsymbol{w}^{T} D^{2} T(\boldsymbol{x}) \boldsymbol{w}^{\prime}=0$ for all $\boldsymbol{w} \in \operatorname{span}\left(\mathcal{V}_{\text {trans }}\right), \boldsymbol{w}^{\prime} \in \mathbb{R}^{3 \times 8}$, and $\boldsymbol{x} \in$ 
$\mathbb{R}^{3 \times 8}$. Consequently, we deduce $\boldsymbol{v}^{T} D^{2} T^{a}\left(\boldsymbol{x}^{0}\right) \boldsymbol{v}=\left(\boldsymbol{v}_{\text {rot }}+\boldsymbol{v}_{\text {bad }}\right)^{T} D^{2} T^{a}\left(\boldsymbol{x}^{0}\right)\left(\boldsymbol{v}_{\text {rot }}+\right.$ $\left.\boldsymbol{v}_{\text {bad }}\right)$.

Moreover, let $A \in \mathbb{R}_{\text {skew }}^{3 \times 3}$ be such that $\boldsymbol{v}_{\text {rot }}=A \boldsymbol{x}^{0}$ and observe that there is a rotation $R_{t} \in S O(3)$ such that $x_{t}^{0}:=R_{t}\left(x^{0}+t \boldsymbol{v}_{\text {rot }}\right)$ is contained in the plane $\mathbb{R}^{2} \times\{0\}$ and one has $\left|R_{t}-(\mathbf{I}-t A)\right|=\mathrm{O}\left(|t A|^{2}\right)$, cf. [35, (3.20)]. (Here $\mathbf{I} \in \mathbb{R}^{3 \times 3}$ denotes the identity matrix.) Consequently, we get $\left|\boldsymbol{x}^{0}-\boldsymbol{x}_{t}^{0}\right|=\mathrm{O}\left(|t A|^{2}\right)$. This implies

$$
\begin{aligned}
T^{a}\left(\boldsymbol{x}^{0}+t\left(\boldsymbol{v}_{\mathrm{rot}}+\boldsymbol{v}_{\mathrm{bad}}\right)\right) & =T^{a}\left(R_{t}\left(\boldsymbol{x}^{0}+t\left(\boldsymbol{v}_{\mathrm{rot}}+\boldsymbol{v}_{\mathrm{bad}}\right)\right)\right)=T^{a}\left(\boldsymbol{x}_{t}^{0}+t R_{t} \boldsymbol{v}_{\mathrm{bad}}\right) \\
& =T^{a}\left(\boldsymbol{x}^{0}+t \boldsymbol{v}_{\mathrm{bad}}+t^{2} \boldsymbol{w}+\mathrm{O}\left(t^{3}\right)\right)
\end{aligned}
$$

for some $\boldsymbol{w} \in \mathbb{R}^{3 \times 8}$ with $|\boldsymbol{w}| \leqq c|A|^{2}$ and the property that the third component of each vector in $\boldsymbol{w}$ is zero. A Taylor expansion and Property 1 of the lemma then yield

$$
\begin{aligned}
T^{a}\left(\boldsymbol{x}^{0}+t\left(\boldsymbol{v}_{\mathrm{rot}}+\boldsymbol{v}_{\mathrm{bad}}\right)\right)= & T^{a}\left(\boldsymbol{x}^{0}\right)+t^{2} D T^{a}\left(\boldsymbol{x}^{0}\right) \boldsymbol{w} \\
& +\frac{t^{2}}{2} \boldsymbol{v}_{\mathrm{bad}}^{T} D^{2} T^{a}\left(\boldsymbol{x}^{0}\right) \boldsymbol{v}_{\mathrm{bad}}+\mathrm{O}\left(t^{3}\right) .
\end{aligned}
$$

As the sum of the angles in the hexagon and at the triple junctions remains invariant under perturbation $w$, we then deduce

$\sum_{j=1}^{3} T^{a}\left(\boldsymbol{x}^{0}+t\left(\boldsymbol{v}_{\mathrm{rot}}+\boldsymbol{v}_{\mathrm{bad}}\right)\right) \cdot \boldsymbol{a}_{j}=8 \pi+\sum_{j=1}^{3} \frac{t^{2}}{2} \boldsymbol{v}_{\mathrm{bad}}^{T} D^{2} T^{a}\left(\boldsymbol{x}^{0}\right) \boldsymbol{v}_{\mathrm{bad}} \cdot \boldsymbol{a}_{j}+\mathrm{O}\left(t^{3}\right)$.

The desired result now follows from the fact that $\sum_{j=1}^{3} \boldsymbol{v}_{\mathrm{bad}}^{T} D^{2} T^{a}\left(\boldsymbol{x}^{0}\right) \boldsymbol{v}_{\mathrm{bad}} \cdot \boldsymbol{a}_{j} \leqq$ $-c\left|\boldsymbol{v}_{\text {bad }}\right|^{2}$ has already been established in the first part of the proof, where we also note that $\left|\boldsymbol{v}_{\text {bad }}\right| \geqq c\left|\boldsymbol{v}-\boldsymbol{v}_{\text {degen }}\right|$ with $\boldsymbol{v}_{\text {degen }}$ being the orthogonal projection of $\boldsymbol{v}$ onto $\operatorname{span}\left(\mathcal{V}_{\text {degen }}\right)$.

For a later purpose we also introduce the mapping $\tilde{E}:[0,2 \pi]^{10} \times[0,+\infty)^{8} \rightarrow$ $\mathbb{R}$ defined by

$$
\tilde{E}(\boldsymbol{y})=\sum_{i=1}^{10} \kappa_{i}^{a} v_{3}\left(y_{i}\right)+\sum_{i=1}^{8} \kappa_{i}^{b} v_{2}\left(y_{i+10}\right)
$$

for $\boldsymbol{y} \in[0,2 \pi]^{10} \times[0,+\infty)^{8}$. Note that $E_{\text {cell }}(\boldsymbol{x})=\tilde{E}(T(\boldsymbol{x}))$ for all $\boldsymbol{x} \in \mathbb{R}^{3 \times 8}$.

Lemma 7.2. (Properties of $\tilde{E}$ ). The mapping $\tilde{E}$ is smooth and there are constants $0<c_{E, 1}<c_{E, 2}$ and $\ell_{0} \in \mathbb{N}$ depending only on $v_{2}$ and $v_{3}$ such that for $\ell \geqq \ell_{0}$

1. $\left(D \tilde{E}\left(T\left(x_{\text {kink }}^{\ell}\right)\right)\right)_{i}=0$ for $i=11, \ldots, 18$,

2. $-c_{E, 2} \ell^{-2} \leqq\left(D \tilde{E}\left(T\left(\boldsymbol{x}_{\mathrm{kink}}^{\ell}\right)\right)\right)_{i} \leqq-c_{E, 1} \ell^{-2}$ for $i=1, \ldots, 10$,

3. $c_{E, 1} \leqq\left(D^{2} \tilde{E}\left(T\left(\boldsymbol{x}_{\mathrm{kink}}^{\ell}\right)\right)\right)_{i i} \leqq c_{E, 2} \quad$ for $i=1, \ldots, 18, \quad\left(D^{2} \tilde{E}\left(T\left(\boldsymbol{x}_{\mathrm{kink}}^{\ell}\right)\right)\right)_{i j}$ $=0$ for $i \neq j$. 
Proof. Property 1 follows from the fact that $T^{b}\left(\boldsymbol{x}_{\text {kink }}^{\ell}\right)=(1, \ldots, 1) \in \mathbb{R}^{8}$ and $v_{2}^{\prime}(1)=0$. To see Property 2, we apply Lemma 6.1 to find $\left(T^{a}\left(\boldsymbol{x}_{\text {kink }}^{\ell}\right)\right)_{i} \in(2 \pi / 3-$ $\left.c_{2} \ell^{-2}, 2 \pi / 3-c_{1} \ell^{-2}\right)$ for $i=1, \ldots, 10$ and the fact that $v_{3} \in C^{2}$ with $v_{3}^{\prime}(2 \pi / 3)=$ $0, v_{3}^{\prime \prime}(2 \pi / 3)>0$. Likewise, Property 3 follows from $v_{2}^{\prime \prime}(1)>0$ and $v_{3}^{\prime \prime}(2 \pi / 3)>0$, respectively.

\subsection{Convexity of the Cell Energy}

The following theorem gives a first property of the Hessian of $E_{\text {cell }}$ at the kink configuration $\boldsymbol{x}_{\text {kink }}^{\ell}$.

Theorem 7.3. (Convexity of $E_{\text {cell }}$ in good directions). Let $0<r<1$. Then there exist $\ell_{0} \in \mathbb{N}$ and a constant $c>0$ depending only on $v_{2}, v_{3}$, and $r$ such that for $\ell \geqq \ell_{0}$ and each $\boldsymbol{v} \in \mathbb{R}^{3 \times 8}$ with

$$
|\boldsymbol{v} \cdot \boldsymbol{w}| \leqq r|\boldsymbol{w}||\boldsymbol{v}| \quad \text { for all } \quad \boldsymbol{w} \in \operatorname{span}\left(\mathcal{V}_{\text {degen }} \cup \mathcal{V}_{\text {bad }}\right)
$$

one has

$$
\boldsymbol{v}^{T} D^{2} E_{\text {cell }}\left(\boldsymbol{x}_{\text {kink }}^{\ell}\right) \boldsymbol{v} \geqq c|\boldsymbol{v}|^{2} .
$$

Proof. First, by the regularity of the mapping $T$, Property 1 in Lemma 7.1, and the fact that $x_{\text {kink }}^{\ell} \rightarrow x^{0}$ for $\ell \rightarrow \infty$, we find $\ell_{0} \in \mathbb{N}$ sufficiently large such that for $\ell \geqq \ell_{0}$ the kernel of $D T\left(\boldsymbol{x}_{\text {kink }}^{\ell}\right)$ has dimension at most 11 . Then we find universal constants $0<c_{1}<c_{2}$ such that for all $\ell \geqq \ell_{0}$, possibly for a larger $\ell_{0}$, we have

$$
\begin{aligned}
& c_{1}|\boldsymbol{v}| \leqq\left|D T\left(\boldsymbol{x}_{\text {kink }}^{\ell}\right) \boldsymbol{v}\right| \leqq c_{2}|\boldsymbol{v}| \text { for all } \boldsymbol{v} \in \operatorname{span}\left(\mathcal{V}_{\text {degen }} \cup \mathcal{V}_{\text {bad }}\right)^{\perp}, \\
& \left|D T\left(\boldsymbol{x}_{\text {kink }}^{\ell}\right) \boldsymbol{v}\right| \leqq c_{2}|\boldsymbol{v}| \ell^{-1} \text { for all } \boldsymbol{v} \in \operatorname{span}\left(\mathcal{V}_{\text {degen }} \cup \mathcal{V}_{\text {bad }}\right)
\end{aligned}
$$

For the second property we used (55). Let be given $\boldsymbol{v} \in \mathbb{R}^{3 \times 8}$ with $|\boldsymbol{v} \cdot \boldsymbol{w}| \leqq$ $r|\boldsymbol{w}||\boldsymbol{v}|$ for all $\boldsymbol{w} \in \operatorname{span}\left(\mathcal{V}_{\text {degen }} \cup \mathcal{V}_{\text {bad }}\right)$. The vector can be written as $\boldsymbol{v}=\boldsymbol{v}_{\text {good }}+$ $\boldsymbol{v}_{\text {good }}^{\perp}$ with two orthogonal vectors $\boldsymbol{v}_{\text {good }}, \boldsymbol{v}_{\text {good }}^{\perp}$ satisfying $\boldsymbol{v}_{\text {good }}^{\perp} \in \operatorname{span}\left(\mathcal{V}_{\text {degen }} \cup\right.$ $\left.\mathcal{V}_{\text {bad }}\right)$ and $\left|\boldsymbol{v}_{\text {good }}\right| \geqq \sqrt{1-r^{2}}|\boldsymbol{v}|$. Consider the mapping $f_{\boldsymbol{v}}: \mathbb{R} \rightarrow \mathbb{R}$ defined by $f_{\boldsymbol{v}}(t)=\tilde{E}\left(T\left(\boldsymbol{x}_{\mathrm{kink}}^{\ell}+t \boldsymbol{v}\right)\right)$. We compute

$$
\begin{aligned}
f_{\boldsymbol{v}}^{\prime}(t)= & D \tilde{E}\left(T\left(\boldsymbol{x}_{\mathrm{kink}}^{\ell}+t \boldsymbol{v}\right)\right)\left(D T\left(\boldsymbol{x}_{\mathrm{kink}}^{\ell}+t \boldsymbol{v}\right) \boldsymbol{v}\right), \\
f_{\boldsymbol{v}}^{\prime \prime}(t)= & \left(D T\left(\boldsymbol{x}_{\mathrm{kink}}^{\ell}+t \boldsymbol{v}\right) \boldsymbol{v}\right)^{T} D^{2} \tilde{E}\left(T\left(\boldsymbol{x}_{\mathrm{kink}}^{\ell}+t \boldsymbol{v}\right)\right)\left(D T\left(\boldsymbol{x}_{\mathrm{kink}}^{\ell}+t \boldsymbol{v}\right) \boldsymbol{v}\right) \\
& +D \tilde{E}\left(T\left(\left(\boldsymbol{x}_{\mathrm{kink}}^{\ell}+t \boldsymbol{v}\right)\right)\left(\boldsymbol{v}^{T} D^{2} T\left(\boldsymbol{x}_{\mathrm{kink}}^{\ell}+t \boldsymbol{v}\right) \boldsymbol{v}\right) .\right.
\end{aligned}
$$

We further observe that by Lemma 7.2, Property 1 and 2, there is a constant $c_{3}$ only depending on $c_{E, 2}$ such that

$$
\left|D \tilde{E}\left(T\left(\boldsymbol{x}_{\text {kink }}^{\ell}\right)\right)\left(\boldsymbol{v}^{T} D^{2} T\left(\boldsymbol{x}_{\text {kink }}^{\ell}\right) \boldsymbol{v}\right)\right| \leqq c_{3}|\boldsymbol{v}|^{2} \ell^{-2} .
$$

Then collecting (57)-(59) and using Property 3 of Lemma 7.2 we derive

$$
\begin{aligned}
\boldsymbol{v}^{T} D^{2} E_{\text {cell }}\left(\boldsymbol{x}_{\text {kink }}^{\ell}\right) \boldsymbol{v} & =f_{\boldsymbol{v}}^{\prime \prime}(0) \\
& \geqq c_{E, 1} c_{1}^{2}\left|\boldsymbol{v}_{\text {good }}\right|^{2}-2 c_{E, 2} c_{2}^{2}\left|\boldsymbol{v}_{\text {good }}\right|\left|\boldsymbol{v}_{\text {good }}^{\perp}\right| \ell^{-1}-c_{3}|\boldsymbol{v}|^{2} \ell^{-2} \\
& \geqq|\boldsymbol{v}|^{2}\left(c_{E, 1} c_{1}^{2}\left(1-r^{2}\right)-2 c_{E, 2} c_{2}^{2} \ell^{-1}-c_{3} \ell^{-2}\right) .
\end{aligned}
$$


For $\ell_{0}$ large enough (depending also on $r$ ) this implies the assertion of the lemma for $\ell \geqq \ell_{0}$.

To investigate the convexity properties in the directions $\mathcal{V}_{\text {bad }}$, we need some further preparations. Recall the reflections introduced in (15). The following lemma is a consequence of Theorem 7.3 and shows that variations in the directions $\mathcal{V}_{\text {good }}$ decrease the energy only to higher order.

Lemma 7.4. (Energy decrease in good directions). There exist $\ell_{0} \in \mathbb{N}$ and $a$ constant $C>0$ depending only on $v_{2}$ and $v_{3}$ such that for $\ell \geqq \ell_{0}$ and each $\boldsymbol{v} \in \operatorname{span}\left(\mathcal{V}_{\text {good }}\right)$

$$
D \tilde{E}\left(T\left(\boldsymbol{x}_{\text {kink }}^{\ell}\right)\right)\left(D T\left(\boldsymbol{x}_{\text {kink }}^{\ell}\right) \boldsymbol{v}\right) \geqq-C|\boldsymbol{v}| \ell^{-3} .
$$

Proof. Let $v \in \operatorname{span}\left(\mathcal{V}_{\text {good }}\right)$ be given and define a perturbation of $v$ by

$$
\boldsymbol{v}^{\prime}=\boldsymbol{v}+s \ell^{-1}|\boldsymbol{v}|\left(0,0, e_{3}, e_{3}, e_{3}, e_{3}, 0,0\right) \in \mathbb{R}^{3 \times 8}
$$

for some universal $s>0$ to be specified below. (Note that the direction $\boldsymbol{v}^{\prime}-\boldsymbol{v}$ increases the third components of the points $x_{3}, \ldots, x_{6}$ of the basic cell). By Property 1 and 2 of Lemma 7.2 and the fact that $\left|\boldsymbol{v}-\boldsymbol{v}^{\prime}\right| \leqq 4 s|\boldsymbol{v}| \ell^{-1}$ it clearly suffices to show

$$
D \tilde{E}\left(T\left(\boldsymbol{x}_{\text {kink }}^{\ell}\right)\right)\left(D T\left(\boldsymbol{x}_{\text {kink }}^{\ell}\right) \boldsymbol{v}^{\prime}\right) \geqq 0 .
$$

To this end, we will show that

$$
\tilde{E}\left(T\left(\boldsymbol{x}_{\mathrm{kink}}^{\ell}+t \boldsymbol{v}^{\prime}\right)\right) \geqq \tilde{E}\left(T\left(\boldsymbol{x}_{\mathrm{kink}}^{\ell}\right)\right)
$$

for all $t>0$ small. Then (61) follows by taking the limit $t \rightarrow 0$.

Consider $\boldsymbol{x}=\boldsymbol{x}_{\mathrm{kink}}^{\ell}+\boldsymbol{t} \boldsymbol{v}^{\prime}$ for $t>0$ small. Possibly after applying a rigid motion we can assume that the second and third components of $\left(x_{1}+x_{7}\right) / 2$ and $\left(x_{2}+x_{8}\right) / 2$ are zero, the points $x_{1}, x_{2}, x_{7}, x_{8}$ lie in the plane $\mathbb{R}^{2} \times\{0\}$ and that the points $x_{3}, x_{4}, x_{5}, x_{6}$ lie in a plane parallel to $\mathbb{R}^{2} \times\{0\}$. (Recall that $\boldsymbol{v}$ induces an in-plane perturbation, i.e., the third component of each vector in $v$ is zero.) We replace $\boldsymbol{x}$ by a symmetrized version as follows.

Define $\boldsymbol{x}_{S_{1}}$ by (16) and note that $E_{\text {cell }}\left(\boldsymbol{x}_{S_{1}}\right)=E_{\text {cell }}(\boldsymbol{x})$. Moreover, it is elementary to see that the third component of each vector in $w_{1}:=x_{S_{1}}-\boldsymbol{x}$ is zero. Consequently, $\boldsymbol{w}_{1}$ is perpendicular to $\mathcal{V}_{\text {bad }}, \mathcal{V}_{\text {trans }}$, and the rotations $\boldsymbol{v}_{2}, \boldsymbol{v}_{3}$. Clearly, as the reflection $S_{1}$ leaves the points $\left(x_{1}+x_{7}\right) / 2$ and $\left(x_{2}+x_{8}\right) / 2$ unchanged, we also have that $\boldsymbol{w}_{1}$ is not parallel to the rotation $\boldsymbol{v}_{1}$. Consequently, by Theorem 7.3 and a continuity argument with $t$ small enough, the mapping $t^{\prime} \mapsto E_{\text {cell }}\left(\boldsymbol{x}+t^{\prime} \boldsymbol{w}_{1}\right)$ is convex on $[0,1]$. This implies for $\boldsymbol{x}^{\prime}=\frac{1}{2}\left(\boldsymbol{x}+\boldsymbol{x}_{S_{1}}\right)$ (see (17a)) that $E_{\text {cell }}\left(\boldsymbol{x}^{\prime}\right) \leqq \frac{1}{2}\left(E_{\text {cell }}(\boldsymbol{x})+E_{\text {cell }}\left(\boldsymbol{x}_{S_{1}}\right)\right)=E_{\text {cell }}(\boldsymbol{x})$.

Likewise, we consider $\boldsymbol{x}_{S_{2}}^{\prime}:=\boldsymbol{x}_{\text {kink }}^{\ell}+S_{2}\left(\boldsymbol{x}^{\prime}-\boldsymbol{x}_{\text {kink }}^{\ell}\right)$ and note that $E_{\text {cell }}\left(\boldsymbol{x}_{S_{2}}^{\prime}\right)=$ $E_{\text {cell }}\left(\boldsymbol{x}^{\prime}\right)$. Similarly as before, the vector $\boldsymbol{w}_{2}:=\boldsymbol{x}_{S_{2}}^{\prime}-\boldsymbol{x}^{\prime}$ is perpendicular to the vectors $\mathcal{V}_{\text {bad }}$ and not parallel to $\mathcal{V}_{\text {degen }}$. Using Theorem 7.3 we get $E_{\text {cell }}(\mathcal{S}(\boldsymbol{x})) \leqq$ $E_{\text {cell }}\left(\boldsymbol{x}^{\prime}\right) \leqq E_{\text {cell }}(\boldsymbol{x})$ for $\mathcal{S}(\boldsymbol{x})=\frac{1}{2}\left(\boldsymbol{x}^{\prime}+\boldsymbol{x}_{S_{2}}^{\prime}\right)($ see $(17 \mathrm{~b}))$. 
By this symmetrization procedure we get that the eight points $\mathcal{S}(\boldsymbol{x})$ are contained in two kinked planes (similarly as $\boldsymbol{x}_{\text {kink }}^{\ell}$ ). We denote the incidence angle of the two planes by $\gamma \leqq \pi$ and note that $\gamma \leqq \gamma_{\ell}$ if the constant $s>0$ in (60) is chosen sufficiently large. The bond lengths satisfy $b_{1}=b_{2}, b_{3}=b_{4}=b_{5}=b_{6}$ and $b_{7}=b_{8}$. For the angles $\varphi_{1}=\varphi_{2}$ and $\varphi_{3}=\ldots=\varphi_{10}$ holds.

Recalling (6) and (56) we find $\alpha$ in a small neighborhood of $\alpha_{\ell}^{\text {us }}$ such that

$$
E_{\text {cell }}(\mathcal{S}(\boldsymbol{x})) \geqq-3+4 v_{3}(\alpha)+2 v_{3}(2 \arcsin (\sin \alpha \sin (\gamma / 2))) .
$$

Now taking $\gamma \leqq \gamma_{\ell}$ into account and recalling that $\alpha_{\ell}^{\text {us }}$ is optimal angle from Proposition 3.1, we find

$$
\begin{aligned}
E_{\text {cell }}(\boldsymbol{x}) & \geqq E_{\text {cell }}(\mathcal{S}(\boldsymbol{x})) \geqq-3+4 v_{3}(\alpha)+2 v_{3}\left(2 \arcsin \left(\sin \alpha \sin \left(\gamma_{\ell} / 2\right)\right)\right) \\
& \geqq-3+4 v_{3}\left(\alpha_{\ell}^{\mathrm{us}}\right)+2 v_{3}\left(2 \arcsin \left(\sin \alpha_{\ell}^{\mathrm{us}} \sin \left(\gamma_{\ell} / 2\right)\right)\right)=E_{\text {cell }}\left(\boldsymbol{x}_{\text {kink }}^{\ell}\right),
\end{aligned}
$$

where the last step follows from (54). This shows (62) and concludes the proof.

The next lemma shows that a perturbation of the angles, which does not change the sum of the angles, essentially does not decrease the energy to first order.

Lemma 7.5. There exist $\ell_{0} \in \mathbb{N}$ and a constant $C>0$ depending only on $v_{2}$ and $v_{3}$ such that for $\ell \geqq \ell_{0}$ and each $\boldsymbol{w}=\left(w_{1}, \ldots, w_{10}\right) \in \mathbb{R}^{10}$ with $\boldsymbol{w} \cdot \boldsymbol{a}_{j}=0$ for $j=1,2,3$ we have

$$
\sum_{i=1}^{10}\left(D \tilde{E}\left(T\left(\boldsymbol{x}_{\text {kink }}^{\ell}\right)\right)\right)_{i} w_{i} \geqq-C|\boldsymbol{w}| \ell^{-3} .
$$

Proof. From Property 2 of Lemma 7.1 we have that the image of the affine mapping $D T^{a}\left(\boldsymbol{x}^{0}\right)$ has dimension 7. Moreover, we have $\left(D T^{a}\left(\boldsymbol{x}^{0}\right) \boldsymbol{v}\right) \cdot \boldsymbol{a}_{j}=0$ for $j=1,2,3$ and all $\boldsymbol{v} \in \mathbb{R}^{3 \times 8}$. Indeed, write $\boldsymbol{v}=\boldsymbol{v}_{\text {good }}+\boldsymbol{v}_{\text {bad }}$ with $\boldsymbol{v}_{\text {good }} \in \operatorname{span}\left(\mathcal{V}_{\text {good }}\right)$ and $v_{\text {bad }} \in \operatorname{span}\left(\mathcal{V}_{\text {degen }} \cup \mathcal{V}_{\text {bad }}\right)$. Note that $D T^{a}\left(\boldsymbol{x}^{0}\right) \boldsymbol{v}=D T^{a}\left(\boldsymbol{x}^{0}\right) \boldsymbol{v}_{\text {good }}$ by Property 1 of Lemma 7.1. For each $t \in \mathbb{R}$ the eight points $\boldsymbol{x}^{0}+t \boldsymbol{v}_{\text {good }}$ are contained in the plane $\mathbb{R}^{2} \times\{0\}$. This implies $T^{a}\left(\boldsymbol{x}^{0}+t \boldsymbol{v}_{\text {good }}\right) \cdot \boldsymbol{a}_{j} \in\{2 \pi, 4 \pi\}$ for all $t \in \mathbb{R}$ and $j=1,2,3$, which gives $\left(D T^{a}\left(\boldsymbol{x}^{0}\right) \boldsymbol{v}_{\text {good }}\right) \cdot \boldsymbol{a}_{j}=0$ for $j=1,2,3$, as desired.

The dimension of the image of $D T^{a}\left(\boldsymbol{x}^{0}\right)$ together with the fact that $\boldsymbol{w} \cdot \boldsymbol{a}_{j}=0$ for $j=1,2,3$ show that there exists a vector $\boldsymbol{v}^{\prime} \in \operatorname{span}\left(\mathcal{V}_{\text {good }}\right)$ such that $D T^{a}\left(\boldsymbol{x}^{0}\right) \boldsymbol{v}^{\prime}=$ $\boldsymbol{w}$. By applying Lemma 7.4 we get

$$
D \tilde{E}\left(T\left(\boldsymbol{x}_{\mathrm{kink}}^{\ell}\right)\right)\left(D T\left(\boldsymbol{x}_{\mathrm{kink}}^{\ell}\right) \boldsymbol{v}^{\prime}\right) \geqq-C^{\prime}\left|\boldsymbol{v}^{\prime}\right| \ell^{-3},
$$

where $C^{\prime}$ is the constant from Lemma 7.4. By a continuity argument and (55) we get $\left|D T\left(\boldsymbol{x}_{\text {kink }}^{\ell}\right)-D T\left(\boldsymbol{x}^{0}\right)\right| \leqq c \ell^{-1}$. This together with Property 2 of Lemma 7.2 shows

$$
D \tilde{E}\left(T\left(\boldsymbol{x}_{\mathrm{kink}}^{\ell}\right)\right)\left(D T\left(\boldsymbol{x}^{0}\right) \boldsymbol{v}^{\prime}\right) \geqq-C\left|\boldsymbol{v}^{\prime}\right| \ell^{-3}
$$

for $C=C\left(C^{\prime}, c_{E, 2}, c\right)$. The fact that $D T^{a}\left(\boldsymbol{x}^{0}\right) \boldsymbol{v}^{\prime}=\boldsymbol{w},\left|\boldsymbol{v}^{\prime}\right| \leqq c|\boldsymbol{w}|$ for a constant $c>0$ (depending on $D T^{a}\left(\boldsymbol{x}^{0}\right)$ ) and Property 1 of Lemma 7.2 conclude the proof. 
We now improve Theorem 7.3 and prove convexity of $E_{\text {cell }}$ at the kink configuration $\boldsymbol{x}_{\text {kink }}^{\ell}$.

Theorem 7.6. (Convexity of $E_{\text {cell }}$ ). Let $0<r<1$. Then there exist $\ell_{0} \in \mathbb{N}$ and a constant $c>0$ depending only on $v_{2}, v_{3}$, and $r$ such that for $\ell \geqq \ell_{0}$ and each $\boldsymbol{v} \in \mathbb{R}^{3 \times 8}$ with

$$
|\boldsymbol{v} \cdot \boldsymbol{w}| \leqq r|\boldsymbol{w}||\boldsymbol{v}| \quad \text { for all } \quad \boldsymbol{w} \in \operatorname{span}\left(\mathcal{V}_{\text {degen }}\right)
$$

one has

$$
\boldsymbol{v}^{T} D^{2} E_{\text {cell }}\left(\boldsymbol{x}_{\text {kink }}^{\ell}\right) \boldsymbol{v} \geqq c|\boldsymbol{v}|^{2} \ell^{-2} .
$$

Proof. As in the proof of Theorem 7.3 we consider the mapping $f_{v}$ as defined before (58). The goal is to show $f_{\boldsymbol{v}}^{\prime \prime}(0) \geqq c|\boldsymbol{v}|^{2} \ell^{-2}$. We write $\boldsymbol{v}=\boldsymbol{v}_{\text {degen }}+\boldsymbol{v}_{\text {bad }}+$ $\boldsymbol{v}_{\text {good }}$ with three orthogonal vectors, where $\boldsymbol{v}_{\text {degen }}+\boldsymbol{v}_{\text {bad }} \in \operatorname{span}\left(\mathcal{V}_{\text {degen }} \cup \mathcal{V}_{\text {bad }}\right)$, $\boldsymbol{v}_{\text {degen }} \in \operatorname{span}\left(\mathcal{V}_{\text {degen }}\right), \boldsymbol{v}_{\text {bad }} \in \operatorname{span}\left(\mathcal{V}_{\text {degen }}\right)^{\perp}$, and $\boldsymbol{v}_{\text {good }} \in \operatorname{span}\left(\mathcal{V}_{\text {degen }} \cup \mathcal{V}_{\text {bad }}\right)^{\perp}$. By assumption we obtain after a short calculation

$$
\left|\boldsymbol{v}_{\text {good }}\right|^{2}+\left|\boldsymbol{v}_{\text {bad }}\right|^{2} \geqq\left(1-r^{2}\right)|\boldsymbol{v}|^{2} .
$$

Set $c_{*}:=\max \left\{2 c_{2} / c_{1},\left(8 c_{3} /\left(c_{E, 1} c_{1}^{2}\right)\right)^{1 / 2}\right\}$ with $c_{1}, c_{2}$ from (57), $c_{3}$ from (59), and $c_{E, 1}$ from Lemma 7.2. First, we suppose $\left|\boldsymbol{v}_{\text {good }}\right| \geqq c_{*}|\boldsymbol{v}| \ell^{-1}$. We use (57) and $\boldsymbol{v}_{\text {good }} \in \operatorname{span}\left(\mathcal{V}_{\text {degen }} \cup \mathcal{V}_{\text {bad }}\right)^{\perp}$ to find

$$
\left|D T\left(\boldsymbol{x}_{\text {kink }}^{\ell}\right) \boldsymbol{v}\right| \geqq c_{1}\left|\boldsymbol{v}_{\text {good }}\right|-c_{2}|\boldsymbol{v}| \ell^{-1} \geqq \frac{c_{1}}{2}\left|\boldsymbol{v}_{\text {good }}\right| .
$$

Then by Property 3 of Lemma 7.2, (58), and (59) we get

$$
\begin{aligned}
f_{\boldsymbol{v}}^{\prime \prime}(0)= & \boldsymbol{v}^{T} D^{2} E_{\text {cell }}\left(\boldsymbol{x}_{\text {kink }}\right) \boldsymbol{v} \geqq\left(D T\left(\boldsymbol{x}_{\text {kink }}^{\ell}\right) \boldsymbol{v}\right)^{T} D^{2} \tilde{E}\left(T\left(\boldsymbol{x}_{\text {kink }}^{\ell}\right)\right)\left(D T\left(\boldsymbol{x}_{\text {kink }}^{\ell}\right) \boldsymbol{v}\right) \\
& -c_{3}|\boldsymbol{v}|^{2} \ell^{-2} \\
\geqq & c_{E, 1}\left|D T\left(\boldsymbol{x}_{\text {kink }}^{\ell}\right) \boldsymbol{v}\right|^{2}-c_{3}|\boldsymbol{v}|^{2} \ell^{-2} \geqq \frac{c_{E, 1} c_{1}^{2}}{4}\left|\boldsymbol{v}_{\text {good }}\right|^{2}-c_{3}|\boldsymbol{v}|^{2} \ell^{-2} \\
\geqq & \frac{c_{E, 1} c_{1}^{2} c_{*}^{2}}{8 \ell^{2}}|\boldsymbol{v}|^{2} .
\end{aligned}
$$

Now suppose $\left|\boldsymbol{v}_{\text {good }}\right|<c_{*}|\boldsymbol{v}| \ell^{-1}$. Since the first term of $f_{\boldsymbol{v}}^{\prime \prime}(0)$ given in (58) is nonnegative, it suffices to consider the second term of $f_{v}^{\prime \prime}(0)$. First, using Property 1 of Lemma 7.2 we have

$$
\sum_{i=11}^{18}\left(D \tilde{E}\left(T\left(\boldsymbol{x}_{\text {kink }}^{\ell}\right)\right)_{i}\left(\boldsymbol{v}^{T} D^{2} T\left(\boldsymbol{x}_{\mathrm{kink}}^{\ell}\right) \boldsymbol{v}\right)_{i}=0 .\right.
$$

Define for brevity $\boldsymbol{w}=\left(\boldsymbol{v}_{\text {degen }}+\boldsymbol{v}_{\text {bad }}\right)^{T} D^{2} T^{a}\left(\boldsymbol{x}_{\text {kink }}^{\ell}\right)\left(\boldsymbol{v}_{\text {degen }}+\boldsymbol{v}_{\text {bad }}\right) \in \mathbb{R}^{10}$ and note that $\left|\boldsymbol{v}_{\text {good }}\right|<c_{*} \ell^{-1}|\boldsymbol{v}|$ implies

$$
\left|\left(\boldsymbol{v}^{T} D^{2} T^{a}\left(\boldsymbol{x}_{\text {kink }}^{\ell}\right) \boldsymbol{v}\right)_{i}-\boldsymbol{w}_{i}\right| \leqq c_{4}|\boldsymbol{v}|^{2} \ell^{-1}, \quad i=1, \ldots, 10
$$


for $c_{4}$ depending on $c_{*}$. By Properties 3 and 4 in Lemma 7.1, (55), and a continuity argument we obtain constants $0<c_{5}<c_{6}$ (depending on $c_{\text {kink }}$ ) such that for $\ell$ sufficiently large

$$
\boldsymbol{w} \cdot \boldsymbol{a}_{j} \leqq c_{6}|\boldsymbol{v}|^{2} \ell^{-1}, \quad j=1,2,3, \quad \sum_{j=1}^{3} \boldsymbol{w} \cdot \boldsymbol{a}_{j} \leqq-c_{5}\left|\boldsymbol{v}_{\mathrm{bad}}\right|^{2}+c_{6}|\boldsymbol{v}|^{2} \ell^{-1}
$$

Consequently, we can find a decomposition $\boldsymbol{w}=\boldsymbol{w}^{\prime}+\boldsymbol{w}^{\prime \prime}$ with the property

$$
\begin{aligned}
& \boldsymbol{w}^{\prime} \cdot \boldsymbol{a}_{j}=0, \quad j=1,2,3, \quad\left|\boldsymbol{w}^{\prime}\right| \leqq c_{7}|\boldsymbol{v}|^{2} \\
& \sum_{i=1}^{10} w_{i}^{\prime \prime} \leqq-c_{5}\left|\boldsymbol{v}_{\mathrm{bad}}\right|^{2}+c_{6}|\boldsymbol{v}|^{2} \ell^{-1}, \quad w_{i}^{\prime \prime} \leqq c_{6}|\boldsymbol{v}|^{2} \ell^{-1}, \quad i=1, \ldots, 10
\end{aligned}
$$

for a universal constant $c_{7}>0$. (Choose, e.g., $w_{3}^{\prime}=w_{3}-\boldsymbol{w} \cdot \boldsymbol{a}_{1}, w_{7}^{\prime}=w_{7}-\boldsymbol{w} \cdot \boldsymbol{a}_{2}$, $w_{9}^{\prime}=w_{9}-\boldsymbol{w} \cdot \boldsymbol{a}_{3}$, and $w_{i}^{\prime}=w_{i}$ else.) Let $I=\left\{i=1, \ldots, 10 \mid \boldsymbol{w}_{i}^{\prime \prime} \leqq 0\right\}$ and note $\sum_{i \in I} \boldsymbol{w}_{i}^{\prime \prime} \leqq \sum_{i=1}^{10} \boldsymbol{w}_{i}^{\prime \prime}$. Then using Property 2 of Lemma 7.2 and Lemma 7.5 we derive

$$
\begin{aligned}
& \sum_{i=1}^{10}\left(D \tilde{E}\left(T\left(\boldsymbol{x}_{\mathrm{kink}}^{\ell}\right)\right)_{i} \boldsymbol{w}_{i}\right. \\
& \quad=\sum_{i=1}^{10}\left(D \tilde{E}\left(T\left(\boldsymbol{x}_{\mathrm{kink}}^{\ell}\right)\right)_{i} \boldsymbol{w}_{i}^{\prime}+\sum_{i \in I}\left(D \tilde{E}\left(T\left(\boldsymbol{x}_{\mathrm{kink}}^{\ell}\right)\right)_{i} \boldsymbol{w}_{i}^{\prime \prime}+\sum_{i \notin I}\left(D \tilde{E}\left(T\left(\boldsymbol{x}_{\mathrm{kink}}^{\ell}\right)\right)_{i} \boldsymbol{w}_{i}^{\prime \prime}\right.\right.\right. \\
& \quad \geqq-C\left|\boldsymbol{w}^{\prime}\right| \ell^{-3}+c_{E, 1} \ell^{-2} \sum_{i \in I}-\boldsymbol{w}_{i}^{\prime \prime}-10 c_{E, 2} c_{6}|\boldsymbol{v}|^{2} \ell^{-3} \\
& \quad \geqq-C c_{7}|\boldsymbol{v}|^{2} \ell^{-3}+c_{E, 1} \ell^{-2}\left(c_{5}\left|\boldsymbol{v}_{\mathrm{bad}}\right|^{2}-c_{6}|\boldsymbol{v}|^{2} \ell^{-1}\right)-10 c_{E, 2} c_{6}|\boldsymbol{v}|^{2} \ell^{-3},
\end{aligned}
$$

where $C$ is the constant from Lemma 7.5. Moreover, again using Lemma 7.2 and (65) we get

$$
\sum_{i=1}^{10} \mid\left(\left.D \tilde{E}\left(T\left(\boldsymbol{x}_{\mathrm{kink}}^{\ell}\right)\right)_{i}\left(\boldsymbol{w}_{i}-\left(\boldsymbol{v}^{T} D^{2} T\left(\boldsymbol{x}_{\mathrm{kink}}^{\ell}\right) \boldsymbol{v}\right)_{i}\right)\left|\leqq 10 c_{E, 2} c_{4}\right| \boldsymbol{v}\right|^{2} \ell^{-3}\right.
$$

We then use (58), (64), and the previous two estimates to find

$$
\begin{aligned}
f_{\boldsymbol{v}}^{\prime \prime}(0) & =\boldsymbol{v}^{T} D^{2} E_{\text {cell }}\left(\boldsymbol{x}_{\text {kink }}\right) \boldsymbol{v} \geqq D \tilde{E}\left(T\left(\boldsymbol{x}_{\text {kink }}^{\ell}\right)\right)\left(\boldsymbol{v}^{T} D^{2} T\left(\boldsymbol{x}_{\text {kink }}^{\ell}\right) \boldsymbol{v}\right) \\
& \geqq c_{E, 1} c_{5}\left|\boldsymbol{v}_{\mathrm{bad}}\right|^{2} \ell^{-2}-c^{\prime}|\boldsymbol{v}|^{2} \ell^{-3}
\end{aligned}
$$

for $c^{\prime}=c^{\prime}\left(C, c_{E, 1}, c_{E, 2}, c_{4}, c_{5}, c_{6}, c_{7}\right)$ large enough. Since $\left|\boldsymbol{v}_{\text {good }}\right|<c_{*}|\boldsymbol{v}| \ell^{-1}$, we get $\left|\boldsymbol{v}_{\text {bad }}\right|^{2} \geqq \frac{1}{2}\left(1-r^{2}\right)|\boldsymbol{v}|^{2}$ for $\ell_{0}$ large enough by (63). Then $f_{v}^{\prime \prime}(0) \geqq c \ell^{-2}|\boldsymbol{v}|^{2}$ follows when we choose $\ell_{0} \in \mathbb{N}$ sufficiently large (depending also on $r$ ). 


\subsection{Proof of Theorem 4.4}

As a last preparation for the proof of Theorem 4.4, we need to investigate how the angles between planes behave under reflection of a configuration (see (15)(17)). Let a center $z_{i, j, k}$ be given and, as before, denote by $\boldsymbol{x} \in \mathbb{R}^{3 \times 8}$ the atoms of the corresponding cell. We introduce the angles between the planes as in Section 4. By $\theta_{l}(\boldsymbol{x})$ we denote the angle between the planes $\left\{x_{1} x_{3} x_{4}\right\}$ and $\left\{x_{1} x_{6} x_{5}\right\}$. By $\theta_{r}(\boldsymbol{x})$ we denote the angle between the planes $\left\{x_{3} x_{4} x_{2}\right\}$ and $\left\{x_{2} x_{5} x_{6}\right\}$. Moreover, we let $\theta_{l}^{\text {dual }}(\boldsymbol{x})=\theta\left(x_{1}\right)$ and $\theta_{r}^{\text {dual }}(\boldsymbol{x})=\theta\left(x_{2}\right)$ with $\theta\left(x_{i}\right), i=1,2$, as defined in (19). Recall also the definition of $\Delta\left(z_{i, j, k}\right)$ in (18).

Lemma 7.7. (Symmetry defect controls angle defect). There exist a universal constant $C>0$ and $\ell_{0} \in \mathbb{N}$, and for each $\ell \geqq \ell_{0}$ there exists $\eta_{\ell}>0$ such that for all $\tilde{\mathcal{F}} \in \mathscr{P}_{\eta_{\ell}}(\mu), \mu \in(2.6,3.1)$, and all centers $z_{i, j, k}$ we have

$$
\begin{aligned}
& \theta_{l}(\mathcal{S}(\boldsymbol{x}))+\theta_{r}(\mathcal{S}(\boldsymbol{x})) \leqq \theta_{l}(\boldsymbol{x})+\theta_{r}(\boldsymbol{x})+C \Delta\left(z_{i, j, k}\right), \\
& \theta_{l}^{\text {dual }}(\mathcal{S}(\boldsymbol{x}))+\theta_{r}^{\text {dual }}(\mathcal{S}(\boldsymbol{x})) \leqq \theta_{l}^{\text {dual }}(\boldsymbol{x})+\theta_{r}^{\text {dual }}(\boldsymbol{x})+C \Delta\left(z_{i, j, k}\right),
\end{aligned}
$$

where $\boldsymbol{x} \in \mathbb{R}^{3 \times 8}$ denotes the position of the atoms in the cell with center $z_{i, j, k}$ and $\mathcal{S}(\boldsymbol{x})$ as in (17b).

We postpone the proof of this lemma to the end of the section and now continue with the proof of Theorem 4.4.

Proof of Theorem 4.4. Let $\tilde{\mathcal{F}} \in \mathscr{P}_{\eta_{\ell}}(\mu)$ be a given configuration, where $\eta_{\ell}$ is specified below, and let $x \in \mathbb{R}^{3 \times 8}$ be the points of one cell as introduced in Section 4 . As usual, possibly after a rigid motion we can assume that the second and third components of $\left(x_{1}+x_{7}\right) / 2,\left(x_{2}+x_{8}\right) / 2$ are zero and the points $x_{4}, x_{5}$ lie in a plane parallel to $\mathbb{R}^{2} \times\{0\}$. We now perform a symmetrization argument as in the proof of Lemma 7.4.

We define $\boldsymbol{x}_{S_{1}}$ by (16). Clearly the vector $\boldsymbol{w}_{1}:=\boldsymbol{x}_{S_{1}}-\boldsymbol{x}$ is perpendicular to $\mathcal{V}_{\text {trans. }}$. Moreover, we have $\left|\boldsymbol{w}_{1} \cdot \boldsymbol{v}_{i}\right| \leqq r\left|\boldsymbol{w}_{1}\right|\left|\boldsymbol{v}_{i}\right|$ for $i=1,2,3$ for a universal constant $r \in(0,1)$. In particular, $r$ is independent of the perturbation $\boldsymbol{x}$. Indeed, for $\boldsymbol{v}_{1}$ and $\boldsymbol{v}_{2}$ this follows from the fact that the points $\left(x_{1}+x_{7}\right) / 2$ and $\left(x_{2}+x_{8}\right) / 2$ are left unchanged. For $v_{3}$ it follows from the assumption that the points $x_{4}, x_{5}$ lie in a plane parallel to $\mathbb{R}^{2} \times\{0\}$.

Consequently, by Theorem 7.6, a continuity argument, and the definition of the the perturbations $\mathscr{P}_{\eta_{\ell}}(\mu)$, the mapping $t \mapsto E_{\text {cell }}\left(\boldsymbol{x}+t \boldsymbol{w}_{1}\right)$ is strictly convex on $[0,1]$ if $\eta_{\ell}$ is chosen small enough (independent of $\left.\boldsymbol{x}\right)$. This implies for $\boldsymbol{x}^{\prime}=\frac{1}{2}(\boldsymbol{x}+$ $\left.\boldsymbol{x}_{S_{1}}\right)($ see $(17 \mathrm{a}))$ that $E_{\text {cell }}\left(\boldsymbol{x}^{\prime}\right)+c \ell^{-2}\left|\boldsymbol{w}_{1}\right|^{2} \leqq \frac{1}{2}\left(E_{\text {cell }}(\boldsymbol{x})+E_{\text {cell }}\left(\boldsymbol{x}_{S_{1}}\right)\right)=E_{\text {cell }}(\boldsymbol{x})$, where $c$ only depends on the constant from Theorem 7.6.

Likewise, we consider $\boldsymbol{x}_{S_{2}}^{\prime}:=\boldsymbol{x}_{\text {kink }}^{\ell}+S_{2}\left(\boldsymbol{x}^{\prime}-\boldsymbol{x}_{\text {kink }}^{\ell}\right)$ and, similarly as before, the vector $\boldsymbol{w}_{2}:=\boldsymbol{x}_{S_{2}}^{\prime}-\boldsymbol{x}^{\prime}$ is perpendicular to $\mathcal{V}_{\text {trans }}$ and satisfies $\left|\boldsymbol{w}_{2} \cdot \boldsymbol{v}_{i}\right| \leqq r\left|\boldsymbol{w}_{2}\right|\left|\boldsymbol{v}_{i}\right|$ for $i=1,2,3$ for a universal constant $r \in(0,1)$. Indeed, for $\boldsymbol{v}_{1}$ and $\boldsymbol{v}_{2}$ this follows as before and for $\boldsymbol{v}_{3}$ it suffices to note that also for the configuration $\boldsymbol{x}^{\prime}=\left(x_{1}^{\prime}, \ldots, x_{8}^{\prime}\right)$ the points $x_{4}^{\prime}, x_{5}^{\prime}$ lie in a plane parallel to $\mathbb{R}^{2} \times\{0\}$. Using again Theorem 7.6 we get $E_{\text {cell }}(\mathcal{S}(\boldsymbol{x}))+c \ell^{-2}\left|\boldsymbol{w}_{2}\right|^{2} \leqq E_{\text {cell }}\left(\boldsymbol{x}^{\prime}\right)$ with $\mathcal{S}(\boldsymbol{x})$ from (17b). Possibly passing to a smaller constant $c>0$ (not relabeled) and using (18), we observe 


$$
E_{\text {cell }}(\mathcal{S}(\mathbf{x}))+c \ell^{-2} \Delta\left(z_{i, j, k}\right) \leqq E_{\text {cell }}(\boldsymbol{x}) .
$$

By this symmetrization procedure we get that the eight points $\mathcal{S}(\mathbf{x})$ satisfy the symmetry conditions stated in (20). In particular, $\tilde{\mu}$ from (20) is here equal to $\left|z_{i, j, k}^{\text {dual }}-z_{i, j-1, k}^{\text {dual }}\right|$, the latter quantity being unchanged after symmetrization since the second and third component of $z_{i, j, k}^{\text {dual }}, z_{i, j-1, k}^{\text {dual }}$ are assumed to be zero. Choose $M^{\ell}$ and $\eta_{\ell}$ small enough such that $\left|\lambda_{1}-1\right|+\left|\lambda_{3}-1\right| \leqq \ell^{-4}$, and $\left|\gamma_{1}-\gamma_{2}\right| \leqq \ell^{-2}$ with $\lambda_{1}, \lambda_{3}, \gamma_{1}, \gamma_{2}$ from (20). This choice of $M^{\ell}$ is possible thanks to Property 2 in Proposition 3.4. Consequently, by Lemma 4.2 we obtain

$$
E_{\text {cell }}(\boldsymbol{x})=E_{\text {cell }}\left(z_{i, j, k}\right) \geqq E_{\widetilde{\mu}, \gamma_{1}, \gamma_{2}}^{\mathrm{sym}}\left(\lambda_{2}, \alpha_{1}, \alpha_{2}\right)+c \ell^{-2} \Delta\left(z_{i, j, k}\right)-c_{0} \ell^{-4}\left(\gamma_{1}-\gamma_{2}\right)^{2} .
$$

Using Property 2 of Proposition 4.3 and (24) we get for $\ell_{0}$ sufficiently large

$$
E_{\text {cell }}\left(z_{i, j, k}\right) \geqq E_{\text {red }}(\tilde{\mu}, \bar{\gamma}, \bar{\gamma})+c \ell^{-2} \Delta\left(z_{i, j, k}\right),
$$

where $\bar{\gamma}=\left(\gamma_{1}+\gamma_{2}\right) / 2$. By Lemma 7.7 we obtain $\bar{\gamma} \leqq \bar{\theta}\left(z_{i, j, k}\right)+C \Delta\left(z_{i, j, k}\right)$, where $\bar{\theta}\left(z_{i, j, k}\right)=\left(\theta_{l}\left(z_{i, j, k}\right)+\theta_{r}\left(z_{i, j, k}\right)+\theta_{l}\left(z_{i, j, k}^{\text {dual }}\right)+\bar{\theta}_{r}\left(z_{i, j-1, k}^{\text {dual }}\right)\right) / 4$. Thus, by the monotonicity of the reduced energy (see Property 3 of Proposition 4.3) and a Taylor expansion for the mapping $\gamma \mapsto E_{\text {red }}(\tilde{\mu}, \gamma, \gamma)$ we get

$$
\begin{aligned}
E_{\mathrm{red}}(\tilde{\mu}, \bar{\gamma}, \bar{\gamma}) & \geqq E_{\mathrm{red}}\left(\tilde{\mu}, \bar{\theta}\left(z_{i, j, k}\right), \bar{\theta}\left(z_{i, j, k}\right)\right)-C \ell^{-3} \Delta\left(z_{i, j, k}\right)+\mathrm{O}\left(\left(\Delta\left(z_{i, j, k}\right)\right)^{2}\right) \\
& \geqq E_{\text {red }}\left(\tilde{\mu}, \bar{\theta}\left(z_{i, j, k}\right), \bar{\theta}\left(z_{i, j, k}\right)\right)-2 C \ell^{-3} \Delta\left(z_{i, j, k}\right)
\end{aligned}
$$

for $C>0$ large enough depending on $v_{3}$, where the last step follows for $\eta_{\ell}$ sufficiently small. The assertion of the theorem now follows for $\ell_{0}$ sufficiently large and $\ell \geqq \ell_{0}$ from (66), (67), and the fact that $\tilde{\mu}=\left|z_{i, j, k}^{\text {dual }}-z_{i, j-1, k}^{\text {dual }}\right|$.

Finally, we give the proof of Lemma 7.7.

Proof of Lemma 7.7. The proof is mainly based on a careful Taylor expansion for the angles under the symmetrization of the atomic positions in the cell, which is induced by the reflections (15). In particular, the argumentation for the angles $\theta_{l}, \theta_{r}$ and the dual angles $\theta_{l}^{\text {dual }}, \theta_{r}^{\text {dual }}$, respectively, is very similar. Therefore, we concentrate on the first inequality in the following.

Let the configuration $\boldsymbol{x}$ be given for a center $z_{i, j, k}$. Let $n_{1}^{l}(\boldsymbol{x})$ and $n_{2}^{l}(\boldsymbol{x})$ be unit normal vectors of the planes $\left\{x_{1} x_{3} x_{4}\right\}$ and $\left\{x_{1} x_{6} x_{5}\right\}$. Likewise, let $n_{1}^{r}(\boldsymbol{x})$ and $n_{2}^{r}(\boldsymbol{x})$ be normal vectors of the planes $\left\{x_{2} x_{4} x_{3}\right\}$ and $\left\{x_{2} x_{5} x_{6}\right\}$. Let $n_{l}(\boldsymbol{x})$ and $n_{r}(\boldsymbol{x})$ be unit vectors perpendicular to $n_{1}^{l}(\boldsymbol{x}), n_{2}^{l}(\boldsymbol{x})$ and $n_{1}^{r}(\boldsymbol{x}), n_{2}^{r}(\boldsymbol{x})$, respectively.

Let $s_{1}^{l}(\boldsymbol{x})$ be a unit vector perpendicular to $n_{l}(\boldsymbol{x}), n_{1}^{l}(\boldsymbol{x})$ and let $s_{2}^{l}(\boldsymbol{x})$ be a unit vector perpendicular to $n_{l}(\boldsymbol{x}), n_{2}^{l}(\boldsymbol{x})$ such that $s_{1}^{l}(\boldsymbol{x}) \cdot s_{2}^{l}(\boldsymbol{x})$ is near -1 . We define $s_{1}^{r}(\boldsymbol{x}), s_{2}^{r}(\boldsymbol{x})$ in a similar fashion. Note that these objects can be chosen to depend smoothly with respect to $\boldsymbol{x}$ and that the angle in (19) can be expressed as

$$
\theta_{k}(\boldsymbol{x})=\arccos \left(s_{1}^{k}(\boldsymbol{x}) \cdot s_{2}^{k}(\boldsymbol{x})\right) \text { for } k=l, r .
$$

We also introduce the mapping

$$
g(\boldsymbol{x})=\arccos \left(s_{1}^{l}(\boldsymbol{x}) \cdot s_{2}^{l}(\boldsymbol{x})\right)+\arccos \left(s_{1}^{r}(\boldsymbol{x}) \cdot s_{2}^{r}(\boldsymbol{x})\right) .
$$


Step I. Recall from the definition in (17), (18) that there are two vectors $\boldsymbol{w}_{1}, \boldsymbol{w}_{2} \in$ $\mathbb{R}^{3 \times 8}$ such that the symmetrized configurations can be expressed as $\boldsymbol{x}^{\prime}=\boldsymbol{x}+\boldsymbol{w}_{1}$ and $\mathcal{S}(\boldsymbol{x})=\boldsymbol{x}^{\prime}+\boldsymbol{w}_{2}$ with

$$
\left|\boldsymbol{w}_{1}\right|^{2}+\left|\boldsymbol{w}_{2}\right|^{2}=\Delta\left(z_{i, j, k}\right)
$$

for a universal constant $C>0$. The goal will be to investigate the Hessian of $g$ and to show

$$
\boldsymbol{w}_{1}^{T} D^{2} g\left(\boldsymbol{x}^{\prime}\right) \boldsymbol{w}_{1}+\boldsymbol{w}_{2}^{T} D^{2} g(\mathcal{S}(\boldsymbol{x})) \boldsymbol{w}_{2} \geqq-C\left(\left|\boldsymbol{w}_{1}\right|^{2}+\left|\boldsymbol{w}_{2}\right|^{2}\right)
$$

for $C>0$ universal. We defer the proof of (70) and first show that the assertion follows from it. We consider the mappings

$$
f_{1}(t)=g\left(\boldsymbol{x}^{\prime}+t \boldsymbol{w}_{1}\right), \quad f_{2}(t)=g\left(\mathcal{S}(\boldsymbol{x})+t \boldsymbol{w}_{2}\right) \quad \text { for } t \in[-1,1]
$$

and observe that $f_{1}(-1)=g(\boldsymbol{x}), f_{2}(-1)=g\left(\boldsymbol{x}^{\prime}\right), f_{1}(1)=g\left(\boldsymbol{x}_{S_{1}}\right), f_{2}(1)=$ $g\left(\boldsymbol{x}_{S_{2}}^{\prime}\right)$, where $\boldsymbol{x}_{S_{1}}=\boldsymbol{x}_{\text {kink }}^{\ell}+S_{1}\left(\boldsymbol{x}-\boldsymbol{x}_{\text {kink }}^{\ell}\right)$ and $\boldsymbol{x}_{S_{2}}^{\prime}=\boldsymbol{x}_{\text {kink }}^{\ell}+S_{2}\left(\boldsymbol{x}^{\prime}-\boldsymbol{x}_{\text {kink }}^{\ell}\right)$, see (15)-(16). Moreover, due to the fact that the symmetrized configurations are obtained by applying the reflections $S_{1}, S_{2}$, see (15), we get that $f_{1}, f_{2}$ are smooth, even functions, in particular, $f_{1}^{\prime}(0)=f_{2}^{\prime}(0)=0$. Thus, by a Taylor expansion we find $\xi \in(-1,0)$ such that

$$
\begin{aligned}
g(\boldsymbol{x})= & f_{1}(-1)=f_{1}(0)-f_{1}^{\prime}(0)+\frac{1}{2} f_{1}^{\prime \prime}(0)-\frac{1}{6} f_{1}^{\prime \prime \prime}(\xi) \geqq g\left(\boldsymbol{x}^{\prime}\right) \\
& +\frac{1}{2} \boldsymbol{w}_{1}^{T} D^{2} g\left(\boldsymbol{x}^{\prime}\right) \boldsymbol{w}_{1}-C\left|\boldsymbol{w}_{1}\right|^{3},
\end{aligned}
$$

where $C>0$ is a universal constant. Indeed, the constant is independent of $\boldsymbol{x}$ as all admissible $\boldsymbol{x}$ lie in a compact neighborhood of $\boldsymbol{x}_{\text {kink }}^{\ell}$ where $g$ is smooth. Applying Taylor once more, we get

$g(\boldsymbol{x}) \geqq g(\mathcal{S}(\boldsymbol{x}))+\frac{1}{2} \boldsymbol{w}_{1}^{T} D^{2} g\left(\boldsymbol{x}^{\prime}\right) \boldsymbol{w}_{1}+\frac{1}{2} \boldsymbol{w}_{2}^{T} D^{2} g(\mathcal{S}(\boldsymbol{x})) \boldsymbol{w}_{2}-C\left|\boldsymbol{w}_{1}\right|^{3}-C\left|\boldsymbol{w}_{2}\right|^{3}$.

Then we conclude for $\eta_{\ell}$ sufficiently small (and thus $\left|\boldsymbol{w}_{1}\right|,\left|\boldsymbol{w}_{2}\right|$ small) by (69)-(70)

$$
g(\boldsymbol{x}) \geqq g(\mathcal{S}(\boldsymbol{x}))-C\left(\left|\boldsymbol{w}_{1}\right|^{2}+\left|\boldsymbol{w}_{2}\right|^{2}\right)=g(\mathcal{S}(\boldsymbol{x}))-C \Delta\left(z_{i, j, k}\right) .
$$

Recalling (68) we obtain the assertion of the lemma.

Step II. It remains to confirm (70). We first concern ourselves with the Hessian of the mapping $f_{1}$ as defined in (71). For $t \in[-1,1]$ we let $u_{j}^{k}(t)=s_{j}^{k}\left(\boldsymbol{x}^{\prime}+t \boldsymbol{w}_{1}\right)$ for $j=1,2$ and $k=l, r$. By a Taylor expansion we obtain

$$
\begin{aligned}
& u_{j}^{k}(t)=s_{j}^{k}\left(\boldsymbol{x}^{\prime}\right)+\left(v_{j}^{1, k}+w_{j}^{1, k}\right) t+\left(v_{j}^{2, k}+w_{j}^{2, k}\right) t^{2}+\mathrm{O}\left(\left|\boldsymbol{w}_{1}\right|^{3} t^{3}\right) \\
& \text { with }\left|u_{j}^{k}(t)\right|=1,
\end{aligned}
$$

where $v_{j}^{1, k}, v_{j}^{2, k}$ are perpendicular to $n_{k}\left(\boldsymbol{x}^{\prime}\right)$ and $w_{j}^{1, k}, w_{j}^{2, k}$ are parallel to $n_{k}\left(\boldsymbol{x}^{\prime}\right)$ such that $\sum_{j=1,2} \sum_{k=l, r}\left(\left|v_{j}^{1, k}\right|+\left|w_{j}^{1, k}\right|\right) \leqq C\left|\boldsymbol{w}_{1}\right|$ and $\sum_{j=1,2} \sum_{k=l, r}\left(\left|v_{j}^{2, k}\right|+\right.$ $\left.\left|w_{j}^{2, k}\right|\right) \leqq C\left|\boldsymbol{w}_{1}\right|^{2}$. (The constant $C$ is again universal as all admissible $\boldsymbol{x}$ lie in a 
compact set and the mappings $s_{j}^{k}$ are smooth.) For $j=1,2$ and $k=l, r$, the two vectors $w_{j}^{1, k}$ and $w_{j}^{2, k}$ are orthogonal to $s_{j}^{k}\left(\boldsymbol{x}^{\prime}\right)$, and taking the first and the second derivative of the constraint $\left|s_{j}^{k}\left(\boldsymbol{x}^{\prime}+t \boldsymbol{w}_{1}\right)\right|^{2}=\left|u_{j}^{k}(t)\right|^{2}=1$ with respect to $t$ yields by an elementary computation

$$
\text { (a) } v_{j}^{1, k} \cdot s_{j}^{k}\left(\boldsymbol{x}^{\prime}\right)=0, \quad \text { (b) }\left|v_{j}^{1, k}\right|^{2}+\left|w_{j}^{1, k}\right|^{2}+2 s_{j}^{k}\left(\boldsymbol{x}^{\prime}\right) \cdot v_{j}^{2, k}=0 .
$$

Then we compute by (71) that

$$
\begin{aligned}
f_{1}(t)= & \sum_{k=l, r} \arccos \left(s_{1}^{k}\left(\boldsymbol{x}^{\prime}\right) \cdot s_{2}^{k}\left(\boldsymbol{x}^{\prime}\right)+\left(v_{1}^{1, k} \cdot s_{2}^{k}\left(\boldsymbol{x}^{\prime}\right)+v_{2}^{1, k} \cdot s_{1}^{k}\left(\boldsymbol{x}^{\prime}\right)\right) t\right. \\
& +\left(v_{1}^{2, k} \cdot s_{2}^{k}\left(\boldsymbol{x}^{\prime}\right)+v_{2}^{2, k} \cdot s_{1}^{k}\left(\boldsymbol{x}^{\prime}\right)+v_{1}^{1, k} \cdot v_{2}^{1, k}+w_{1}^{1, k} \cdot w_{2}^{1, k}\right) t^{2} \\
& \left.+\mathrm{O}\left(\left|\boldsymbol{w}_{1}\right|^{3} t^{3}\right)\right) .
\end{aligned}
$$

A Taylor expansion and the fact that $f_{1}$ is even yield $f_{1}(t)-f_{1}(0)=f_{1}^{\prime \prime}(0) t^{2} / 2+$ $\mathrm{O}\left(\left|\boldsymbol{w}_{1}\right|^{3} t^{3}\right)$. More precisely, we get recalling $s_{1}^{k}\left(\boldsymbol{x}^{\prime}\right) \cdot s_{2}^{k}\left(\boldsymbol{x}^{\prime}\right)=\cos \left(\theta_{k}\left(\boldsymbol{x}^{\prime}\right)\right)$ for $k=l, r$

$$
\begin{aligned}
f_{1}(t)-f_{1}(0)= & \sum_{k=l, r} \arccos ^{\prime}\left(\cos \left(\theta_{k}\left(\boldsymbol{x}^{\prime}\right)\right)\right)\left(v_{1}^{2, k} \cdot s_{2}^{k}\left(\boldsymbol{x}^{\prime}\right)+v_{2}^{2, k} \cdot s_{1}^{k}\left(\boldsymbol{x}^{\prime}\right)\right. \\
& \left.+v_{1}^{1, k} \cdot v_{2}^{1, k}+w_{1}^{1, k} \cdot w_{2}^{1, k}\right) t^{2} \\
& +\sum_{k=l, r} \frac{1}{2} \arccos ^{\prime \prime}\left(\cos \left(\theta_{k}\left(\boldsymbol{x}^{\prime}\right)\right)\right)\left(v_{1}^{1, k} \cdot s_{2}^{k}\left(\boldsymbol{x}^{\prime}\right)\right. \\
& \left.+v_{2}^{1, k} \cdot s_{1}^{k}\left(\boldsymbol{x}^{\prime}\right)\right)^{2} t^{2}+\mathrm{O}\left(\left|\boldsymbol{w}_{1}\right|^{3} t^{3}\right) .
\end{aligned}
$$

We get $\left|v_{1}^{1, k} \cdot s_{2}^{k}\left(\boldsymbol{x}^{\prime}\right)\right|=\left|v_{1}^{1, k}\right| \sin \left(\theta_{k}\left(\boldsymbol{x}^{\prime}\right)\right)$ by (73)(a). This together with (73)(b) and $\left|v_{1}^{2, k}\right| \leqq C\left|\boldsymbol{w}_{1}\right|^{2}$ yields, for $k=l, r$,

$$
\begin{aligned}
v_{1}^{2, k} \cdot s_{2}^{k}\left(\boldsymbol{x}^{\prime}\right)= & \left(\left(v_{1}^{2, k} \cdot s_{1}^{k}\left(\boldsymbol{x}^{\prime}\right)\right) s_{1}^{k}\left(\boldsymbol{x}^{\prime}\right)+\left|v_{1}^{1, k}\right|^{-2}\left(v_{1}^{2, k} \cdot v_{1}^{1, k}\right) v_{1}^{1, k}\right) \cdot s_{2}^{k}\left(\boldsymbol{x}^{\prime}\right) \\
= & -\frac{1}{2}\left(\left|v_{1}^{1, k}\right|^{2}+\left|w_{1}^{1, k}\right|^{2}\right) \cos \left(\theta_{k}\left(\boldsymbol{x}^{\prime}\right)\right) \\
& +\left|v_{1}^{1, k}\right|^{-2}\left(v_{1}^{2, k} \cdot v_{1}^{1, k}\right)\left(v_{1}^{1, k} \cdot s_{2}^{k}\left(\boldsymbol{x}^{\prime}\right)\right) \\
\leqq & -\frac{1}{2}\left(\left|v_{1}^{1, k}\right|^{2}+\left|w_{1}^{1, k}\right|^{2}\right) \cos \left(\theta_{k}\left(\boldsymbol{x}^{\prime}\right)\right)+C \sin \left(\theta_{k}\left(\boldsymbol{x}^{\prime}\right)\right)\left|\boldsymbol{w}_{1}\right|^{2}
\end{aligned}
$$

and repeating the same calculation for $v_{2}^{2, k}$, we derive, for $k=l, r$,

$$
\begin{aligned}
\left(v_{1}^{2, k} \cdot s_{2}^{k}\left(\boldsymbol{x}^{\prime}\right)+v_{2}^{2, k} \cdot s_{1}^{k}\left(\boldsymbol{x}^{\prime}\right)\right) \leqq & \sum_{j=1,2}-\frac{1}{2}\left(\left|v_{j}^{1, k}\right|^{2}+\left|w_{j}^{1, k}\right|^{2}\right) \cos \left(\theta_{k}\left(\boldsymbol{x}^{\prime}\right)\right) \\
& +C \sin \left(\theta_{k}\left(\boldsymbol{x}^{\prime}\right)\right)\left|\boldsymbol{w}_{1}\right|^{2} .
\end{aligned}
$$

Note that $v_{1}^{1, k} \cdot v_{2}^{1, k}=\left|v_{1}^{1, k}\right|\left|v_{2}^{1, k}\right| q \cos \left(\theta_{k}\left(\boldsymbol{x}^{\prime}\right)\right)$ for $q \in\{-1,1\}$ by (73)(a). An elementary computation then yields 


$$
\left(v_{1}^{1, k} \cdot s_{2}^{k}\left(\boldsymbol{x}^{\prime}\right)+v_{2}^{1, k} \cdot s_{1}^{k}\left(\boldsymbol{x}^{\prime}\right)\right)^{2}=\sin ^{2}\left(\theta_{k}\left(\boldsymbol{x}^{\prime}\right)\right)\left(\left|v_{1}^{1, k}\right|-q\left|v_{2}^{1, k}\right|\right)^{2} .
$$

Combining (74)-(76) and using that $\arccos ^{\prime}(x)=-\left(1-x^{2}\right)^{-1 / 2}$ and that $\arccos ^{\prime \prime}(x)=-x\left(1-x^{2}\right)^{-3 / 2}$, we find

$$
\begin{aligned}
f_{1}(t) & -f_{1}(0) \\
\geqq & \sum_{k=l, r}-\sin \left(\theta_{k}\left(\boldsymbol{x}^{\prime}\right)\right)^{-1}\left(\sum_{j=1,2}-\frac{1}{2}\left(\left|v_{j}^{1, k}\right|^{2}+\left|w_{j}^{1, k}\right|^{2}\right) \cos \left(\theta_{k}\left(\boldsymbol{x}^{\prime}\right)\right)\right. \\
& +C \sin \left(\theta_{k}\left(\boldsymbol{x}^{\prime}\right)\right)\left|\boldsymbol{w}_{1}\right|^{2} \\
& \left.+w_{1}^{1, k} \cdot w_{2}^{1, k}+\left|v_{1}^{1, k}\right|\left|v_{2}^{1, k}\right| q \cos \left(\theta_{k}\left(\boldsymbol{x}^{\prime}\right)\right)\right) t^{2} \\
- & \frac{1}{2} \cos \left(\theta_{k}\left(\boldsymbol{x}^{\prime}\right)\right)\left(1-\cos ^{2}\left(\theta_{k}\left(\boldsymbol{x}^{\prime}\right)\right)\right)^{-3 / 2} \sin ^{2}\left(\theta_{k}\left(\boldsymbol{x}^{\prime}\right)\right)\left(\left|v_{1}^{1, k}\right|-q\left|v_{2}^{1, k}\right|\right)^{2} t^{2} \\
& +\mathrm{O}\left(\left|\boldsymbol{w}_{1}\right|^{3} t^{3}\right) \\
= & \sum_{k=l, r}-\sin \left(\theta_{k}\left(\boldsymbol{x}^{\prime}\right)\right)^{-1}\left(\sum_{j=1,2}-\frac{1}{2}\left|w_{j}^{1, k}\right|^{2} \cos \left(\theta_{k}\left(\boldsymbol{x}^{\prime}\right)\right)+w_{1}^{1, k} \cdot w_{2}^{1, k}\right) t^{2} \\
& -C\left|\boldsymbol{w}_{1}\right|^{2} t^{2}+\mathrm{O}\left(\left|\boldsymbol{w}_{1}\right|^{3} t^{3}\right) \\
\geqq & \sum_{k=l, r}-\sin \left(\theta_{k}\left(\boldsymbol{x}^{\prime}\right)\right)^{-1}\left(\sum_{j=1,2} \frac{1}{2}\left|w_{j}^{1, k}\right|^{2}+w_{1}^{1, k} \cdot w_{2}^{1, k}\right) t^{2} \\
& -C\left|\boldsymbol{w}_{1}\right|^{2} t^{2}+\mathrm{O}\left(\left|\boldsymbol{w}_{1}\right|^{3} t^{3}\right) .
\end{aligned}
$$

In the last step we used that $\cos \theta \geqq-1$. Before we proceed let us note that the same computation can be repeated for the second mapping $f_{2}$ defined in (71): considering an expansion as in (72) with $s_{j}^{k}(\mathcal{S}(\boldsymbol{x}))$ in place of $s_{j}^{k}\left(\boldsymbol{x}^{\prime}\right)$ and indicating the vectors by $\hat{v}_{j}^{i, k}$ and $\hat{w}_{j}^{i, k}$ (perpendicular and parallel to $n_{k}(\mathcal{S}(\boldsymbol{x})$ ), respectively) we also obtain

$$
\begin{aligned}
& f_{2}(t)-f_{2}(0) \\
& \geqq \sum_{k=l, r}-\frac{1}{\sin \left(\theta_{k}(\mathcal{S}(\boldsymbol{x}))\right)}\left(\sum_{j=1,2} \frac{1}{2}\left|\hat{w}_{j}^{1, k}\right|^{2}+\hat{w}_{1}^{1, k} \cdot \hat{w}_{2}^{1, k}\right) t^{2} \\
& \quad-C\left|\boldsymbol{w}_{2}\right|^{2} t^{2}+\mathrm{O}\left(\left|\boldsymbol{w}_{2}\right|^{3} t^{3}\right) .
\end{aligned}
$$

Step III. We now investigate (77)-(78) in more detail. Consider first $f_{1}$. Due to the symmetry of the setting induced by the reflection $S_{1}$ (recall (15)) we find $u_{1}^{k}(t) \cdot n_{k}\left(\boldsymbol{x}^{\prime}\right)=u_{2}^{k}(-t) \cdot n_{k}\left(\boldsymbol{x}^{\prime}\right)$ for $k=l, r$. In particular, taking the derivative in $t$ and using (73)(a), this implies $w_{1}^{1, k}=-w_{2}^{1, k}$. Then by (77) we obtain

$$
f_{1}(t)-f_{1}(0) \geqq-C\left|\boldsymbol{w}_{1}\right|^{2} t^{2}+\mathrm{O}\left(\left|\boldsymbol{w}_{1}\right|^{3} t^{3}\right),
$$


and therefore taking $t \rightarrow 0$ we get $\boldsymbol{w}_{1}^{T} D^{2} g\left(\boldsymbol{x}^{\prime}\right) \boldsymbol{w}_{1} \geqq-C\left|\boldsymbol{w}_{1}\right|^{2}$, which establishes the first part of (70). Now consider $f_{2}$. Notice that one can show $\hat{w}_{1}^{1, k}=\hat{w}_{2}^{1, k}$ for $k=l, r$ by symmetry, i.e., we cannot repeat the same argument as for $f_{1}$. However, in this case we can show

$$
\left|\hat{w}_{1}^{1, l}\right|+\left|\hat{w}_{1}^{1, r}\right|+\left|\hat{w}_{2}^{1, l}\right|+\left|\hat{w}_{2}^{1, r}\right| \leqq C\left|\boldsymbol{w}_{2}\right| \ell^{-1} .
$$

Once this is proved, the assertion follows. Indeed, due to symmetry of $\mathcal{S}(\boldsymbol{x})$ we observe that $\theta_{l}(\mathcal{S}(\boldsymbol{x}))=\theta_{r}(\mathcal{S}(\boldsymbol{x}))$, denoted by $\varphi$ in the following. Recalling (54) and the fact that $\mathcal{S}(\boldsymbol{x})$ is near $\boldsymbol{x}_{\mathrm{kink}}^{\ell}$, we get $\varphi \leqq \pi-c \ell^{-1}$ and $\sin (\varphi) \geqq c \ell^{-1}$ for some $c>0$. Then by (78) we have

$$
f_{2}(t)-f_{2}(0) \geqq-C\left|\boldsymbol{w}_{2}\right|^{2} t^{2}-C \ell \cdot\left|\boldsymbol{w}_{2}\right|^{2} \ell^{-2} t^{2}+\mathrm{O}\left(\left|\boldsymbol{w}_{2}\right|^{3} t^{3}\right),
$$

which shows the second part of (70).

Let us finally show (79). Recall the definition of the unit normal vectors $n_{1}^{k}(\boldsymbol{x}), n_{2}^{k}(\boldsymbol{x})$, and $n_{k}(\boldsymbol{x})$ introduced before (68) for $k=l, r$. Observe that by symmetry reasons we have $n_{k}(\mathcal{S}(\boldsymbol{x}))= \pm e_{1}$ and $\left|n_{j}^{k}(\mathcal{S}(\boldsymbol{x})) \cdot e_{2}\right|=\sin \left(\frac{\pi-\varphi}{2}\right)$ for $j=1,2, k=l, r$. Then a continuity argument gives $\left|n_{k}\left(\boldsymbol{x}^{\prime}\right) \cdot e_{3}\right| \leqq C\left|\boldsymbol{w}_{2}\right|$ and $\left|n_{j}^{k}\left(\boldsymbol{x}^{\prime}\right) \cdot e_{2}\right| \leqq \sin \left(\frac{\pi-\varphi}{2}\right)+C\left|\boldsymbol{w}_{2}\right|$ for $k=l, r$ and $j=1,2$. Moreover, as $\boldsymbol{x}^{\prime}$ is invariant under the reflection $S_{1}$ (recall (15)), we get $n_{k}\left(\boldsymbol{x}^{\prime}\right) \cdot e_{2}=0$. By the definition of $s_{j}^{k}\left(\boldsymbol{x}^{\prime}\right)$ this implies

$$
\begin{aligned}
\left|s_{j}^{k}\left(\boldsymbol{x}^{\prime}\right) \cdot e_{1}\right| & =\left|\left(n_{k}\left(\boldsymbol{x}^{\prime}\right) \times n_{j}^{k}\left(\boldsymbol{x}^{\prime}\right)\right) \cdot e_{1}\right|=\left|n_{k}\left(\boldsymbol{x}^{\prime}\right) \cdot e_{3}\right|\left|n_{j}^{k}\left(\boldsymbol{x}^{\prime}\right) \cdot e_{2}\right| \\
& \leqq C \sin \left(\frac{\pi-\varphi}{2}\right)\left|\boldsymbol{w}_{2}\right|+C\left|\boldsymbol{w}_{2}\right|^{2} .
\end{aligned}
$$

For a small enough perturbation parameter $\eta_{\ell}$ we get $\left|\boldsymbol{w}_{2}\right| \leqq \ell^{-1}$ and thus $\mid s_{j}^{k}\left(\boldsymbol{x}^{\prime}\right)$. $e_{1}|\leqq C| \boldsymbol{w}_{2} \mid \ell^{-1} \operatorname{since} \sin \left(\frac{\pi-\varphi}{2}\right) \leqq c \ell^{-1}$ by (54). As $s_{j}^{k}\left(\boldsymbol{x}^{\prime}\right) \cdot e_{1}=s_{j}^{k}(\mathcal{S}(\boldsymbol{x})) \cdot e_{1}-$ $\hat{w}_{j}^{1, k}+\mathrm{O}\left(\left|\boldsymbol{w}_{2}\right|^{2}\right)=-\hat{w}_{j}^{1, k}+\mathrm{O}\left(\left|\boldsymbol{w}_{2}\right|^{2}\right)\left(\operatorname{see}(72)\right.$ and use the fact that $s_{j}^{k}(\mathcal{S}(\boldsymbol{x})) \cdot e_{1}=$ $0)$, this shows (79) and concludes the proof.

Acknowledgements. Open access funding provided by Austrian Science Fund (FWF). M.F. acknowledges support from the Alexander von Humboldt Stiftung. E.M. acknowledges support from the Austrian Science Fund (FWF) project M 1733-N20. P. P. acknowledges support from the Austrian Science Fund (FWF) project P 29681, and from the Vienna Science and Technology Fund (WWTF), the City of Vienna, and the Berndorf Private Foundation through Project MA16-005. U.S. acknowledges support from the Austrian Science Fund (FWF) projects P 27052, I 2375, and F 65 and from the Vienna Science and Technology Fund (WWTF) through project MA14-009. The authors would like to acknowledge the kind hospitality of the Erwin Schrödinger International Institute for Mathematics and Physics, where part of this research was developed under the frame of the thematic program Nonlinear Flows.

\section{Compliance with ethical standards}

Conflict of interest The authors declare that they have no conflict of interest.

Open Access This article is distributed under the terms of the Creative Commons Attribution 4.0 International License (http://creativecommons.org/licenses/by/4.0/), which permits unrestricted use, distribution, and reproduction in any medium, provided you give appropriate credit to the original author(s) and the source, provide a link to the Creative Commons license, and indicate if changes were made. 


\section{References}

1. Agrawal, P.M., Sudalayandi, B.S., RafF, L.M., Komandur, R.: Molecular dynamics (MD) simulations of the dependence of $\mathrm{C}-\mathrm{C}$ bond lengths and bond angles on the tensile strain in single-wall carbon nanotubes (SWCNT). Comput. Mater. Sci. 41, 450-456, 2008

2. Allinger, N.L.: Molecular Structure: Understanding Steric and Electronic Effects from Molecular Mechanics. Wiley, New York, 2010

3. Arroyo, M., Belytschko, T.: Continuum mechanics modeling and simulation of carbon nanotubes. Meccanica, 40, 455-469, 2005

4. Bajaj, C., Favata, A., Podio-Guidugli, P.: On a nanoscopically-informed shell theory of single-wall carbon nanotubes. Eur. J. Mech. A Solids, 42, 137-157, 2013

5. Braides, A., Lew, A., Ortiz, M.: Effective cohesive behavior of layers of interatomic planes. Arch. Ration. Mech. Anal. 180, 151-182, 2006

6. Brenner, D.W.: Empirical potential for hydrocarbons for use in stimulating the chemical vapor deposition of diamond films. Phys. Rev. B, 42, 9458-9471, 1990

7. Brook, B.R., Bruccoleri, R.E., Olafson, B.D., States, D.J., Swaminathan, S., KARPLUS, M.: CHARMM: a program for macromolecular energy, minimization, and dynamics calculations. J. Comput. Chem. 4, 187-217, 1983

8. Budyka, M.E., Zyubina, T.S., Ryabenko, A.G., Lin, S.H., Mebel, A.M.: Bond lengths and diameters of armchair single-walled carbon nanotubes. Chem. Phys. Lett. 407, 266-271, 2005

9. CAO, G.X., CHEN, X.: The effects of chirality and boundary conditions on the mechanical properties of single-wall carbon nanotubes. Int. J. Solid. Struct. 44, 5447-5465, 2007

10. Charlier, J.-C., Lambin, Ph.: Electronic structure of carbon nanotubes with chiral symmetry. Phys. Rev. B, 57, R15037, 1998

11. Clark, M., Cramer III, R.D., Van Opdenbosch, N.: Validation of the general purpose tripos 5.2 force field. J. Comput. Chem. 10 982-1012, 1989

12. Clayden, J., Greeves, N., Warren, S.G.: Organic Chemistry. Oxford University Press, Oxford, 2012

13. Conti, S., Dolzmann, G., Kirchheim, B., Müller, S.: Sufficient conditions for the validity of the Cauchy-Born rule close to $S O($ n). J. Eur. Math. Soc. (JEMS), 8, 515-530, 2006

14. Cox, B.J., HiLl, J.M.: Exact and approximate geometric parameters for carbon nanotubes incorporating curvature. Carbon, 45, 1453-1462, 2007

15. Cox, B.J., HiLl, J.M.: Geometric structure of ultra-small carbon nanotubes. Carbon, 46, 711-713, 2008

16. Davoli, E., Piovano, P., Stefanelli, U.: Wulff shape emergence in graphene. Math. Models Methods Appl. Sci. 26(12), 2277-2310, 2016

17. Demczyk, B.G., et al.: Direct mechanical measurement of the tensile strength and elastic modulus of multiwalled carbon nanotubes. Mater. Sci. Eng. A334, 173-178, 2002

18. Dresselhaus, M.S., Dresselhaus, G., Saito, R.: Carbon fibers based on $\mathrm{C}_{60}$ ad their symmetry. Phys. Rev. B, 45(11), 6234-6242, 1992

19. Dresselhaus, M.S., Dresselhaus, G., Saito, R.: Physics of carbon nanotubes. Carbon, 33, 883-891, 1995

20. LI, W.E.D.: On the crystallization of 2D hexagonal lattices, Commun. Math. Phys. 286(3), 1099-1140, 2009

21. Ming, W.E.P.: Cauchy-Born rule and the stability of crystalline solids: dynamic problems. Acta Math. Appl. Sin. Engl. Ser. 23, 529-550, 2007

22. Ming, W.E.P.: Cauchy-Born rule and the stability of crystalline solids: static problems. Arch. Ration. Mech. Anal. 183, 241-297, 2007

23. Jorio, A., Dresselhaus, G., Dresselhaus, M.S. (Eds.).: Carbon Nanotubes Advanced Topics in the Synthesis, Structure, Properties and Applications, Topics in Applied Physics, vol. 111. Springer, New York, 2011 
24. El Kass, D., Monneau, R.: Atomic to continuum passage for nanotubes: a discrete Saint-Venant principle and error estimates. Arch. Ration. Mech. Anal. 213, 25-128, 2014

25. Ericksen, J.L.: On the Cauchy-Born rule. Math. Mech. Solids, 13, 199-220, 2008

26. ERICKSEN, J.L.: The Cauchy and Born hypotheses for crystals. In: Phase Transformations and Material Instabilities in Solids (Madison, Wis., 1983), vol. 52, pp. 61-77, Publ. Math. Res. Center Univ. Wisconsin. Academic Press, Orlando, 1984

27. Farmer, B., Esedō̄lu, S., Smereka, P.: Crystallization for a Brenner-like potential. Commun. Math. Phys. 349, 1029-1061, 2017

28. Favata, A., Podio-Guidugli, P.: A new CNT-oriented shell theory. Eur. J. Mech. A/Solids, 35, 75-96, 2012

29. Favata, A., Micheletti, A., Podio-Guidugli, P.: A nonlinear theory of prestressed elastic stick-and-spring structures. J. Eng. Sci. 80, 4-20, 2014

30. Favata, A., Podio-Guidugli, P.: A shell theory for carbon nanotube of arbitrary chirality. In: Shell and Membrane Theories in Mechanics and Biology. Advanced Structured Materials, vol. 45, pp. 155-167. Springer, Cham, 2015

31. Favata, A., Micheletti, A., Podio-Guidugli, P., Pugno, N.M.: Geometry and selfstress of single-wall carbon nanotubes and graphene via a discrete model based on a 2nd-generation REBO potential. J. Elast., 125, 1-37, 2016

32. Friedrich, M., Piovano, P., Stefanelli, U.: The geometry of $C_{60}$ : a rigorous approach via molecular mechanics. SIAM J. Appl. Math. 76, 2009-2029, 2016

33. Friedrich, M., Schmidt, B.: An atomistic-to-continuum analysis of crystal cleavage in a two-dimensional model problem. J. Nonlinear Sci. 24, 145-183, 2014

34. Friedrich, M., Schmidt, B.: An analysis of crystal cleavage in the passage from atomistic models to continuum theory. Arch. Ration. Mech. Anal. 217, 263-308, 2015

35. Friesecke, G., James, R.D., Müller, S.: A theorem on geometric rigidity and the derivation of nonlinear plate theory from three-dimensional elasticity. Commun. Pure Appl. Math. 55, 1461-1506, 2002

36. Friesecke, G., Theil, F.: Validity and failure of the Cauchy-Born hypothesis in a two-dimensional mass-spring lattice. J. Nonlinear Sci. 12, 445-478, 2002

37. Geim, A.K., Novoselov, K.S.: The rise of graphene. Nat. Mater. 6, 183-191, 2007

38. van Gunsteren, W.F., Berendsen, H.J.C.: Groningen Molecular Simulation (GROMOS) Library Manual, BIOMOS b.v., Groningen, 1987

39. Gupta, A., Sakthivela, T., Seal, S.: Recent development in 2D materials beyond graphene. Progr. Mat. Sci., 73, 44-126, 2015

40. Han, F., Azdoud, Y., Lubineau, G.: Computational modeling of elastic properties of carbon nanotube/polymer composites with interphase regions. Part I: micro-structural characterization and geometric modeling. Comput. Mater. Sci. 81, 641-651, 2014

41. IIJima, S.: Helical microtubules of graphitic carbon. Nature, 354, 56-58, 1991

42. James, R.D.: Objective structures. J. Mech. Phys. Solids, 54, 2354-2390, 2006

43. Jiang, H., Zhang, P., Liu, B., Huans, Y., Geubelle, P.H., Gao, H., Hwang, K.C.: The effect of nanotube radius on the constitutive model for carbon nanotubes. Comput. Mater. Sci. 28, 429-442, 2003

44. Jindal, V.K., ImTANI, A.N.: Bond lengths of armchair single-walled carbon nanotubes and their pressure dependence. Comput. Mater. Sci. 44, 156-162, 2008

45. Jishi, R.A., Dresselhaus, M.S., Dresselhaus, G.: Symmetry properties and chiral carbon nanotubes. Phys. Rev. B, 47, 166671-166674, 1993

46. Kanamitsu, K., Saito, S.: Geometries, electronic properties, and energetics of isolated single-walled carbon nanotubes. J. Phys. Soc. Jpn., 71, 2:483-486, 2002

47. Krishnan, A., Dujardin, E., Ebbesen, T.W., Yianilos, P.N., Treacy, M.M.J.: Young's modulus of single-walled nanotubes. Phys. Rev. B, 58, 14013-14019, 1998

48. Kroto, H.W., Heath, J.R., O'Brien, S.C., Curl, R.F., Smalley, R.E.: C 60: buckminsterfullerene. Nature, 318, 162-163, 1985

49. Квото, H.W.: The stability of the fullerenes $C_{n}$, with $n=24,28,32,36,50,60$ and 70. Nature, 329, 529-531, 1987 
50. Kurti, J., Zolyomi, V., Kertesz, M., Sun, G.: The geometry and the radial breathing model of carbon nanotubes: Beyond the ideal behaviour. New J. Phys. 5, 1-21, 2003

51. Lazzaroni, G., Stefanelli, U.: Chain-like ground states in three dimensions. In preparation, 2017

52. LEE, R.K.F., Cox, B.J., HiLl, J.M.: General rolled-up and polyhedral models for carbon nanotubes. Fuller. Nanotub. Carbon Nanostruct., 19, 726-748, 2011

53. Lewars, E.G.: Computational Chemistry, 2nd edn. Springer, New York, 2011

54. LI, X., YANG, W., LiU, B.: Bending induced rippling and twisting of multiwalled carbon nanotubes. Phys. Rev. Lett. 98, 205502-205505, 2007

55. Mainini, E., Murakawa, H., Piovano, P., Stefanelli, U.: Carbon-nanotube geometries: analytical and numerical results. Discrete Contin. Dyn. Syst. Ser. S, 10, 141-160, 2017

56. Mainini, E., Murakawa, H., Piovano, P., Stefanelli, U.: Carbon-nanotube geometries as optimal configurations. Multiscale Model. Simul., 15, (4), 1448-1471, 2017

57. Mainini, E., Stefanelli, U.: Crystallization in carbon nanostructures. Commun. Math. Phys. 328(2), 545-571, 2014

58. Mannix, A.J., Kiraly, B., Hersma, M.C., Guisiger, N.P.: Synthesis and chemistry of elemental 2D materials. Nat. Rev. Chem. 1, 14, 2017

59. Mas-Ballesté, R., Gómez-Navarro, C., Gómez-Herrero, J., Zamora, F.: 2D materials: to graphene and beyond. Nanoscale, 3, 20, 2011

60. Mayo, S.L., Olafson, B.D., Goddard, W.A.: DREIDING: a generic force field for molecular simulations. J. Phys. Chem. 94, 8897-8909, 1990

61. Morris, J.E., INIEWSKI, K.: Graphene, carbon nanotubes, and nanostructures: techniques and applications, CRC Press, Boca Raton, 2013

62. Novoselov, K.S. et al.: Two-dimensional gas of massless Dirac fermions in graphene. Nature, 438, 197-200, 2005

63. Poncharal, P., Wang, Z.L., Ugarte, D., De Heer, W.A.: Electrostatic deflections and electro-mechanical resonances of carbon nanotubes. Science, 283, 1513-1516, 1999

64. Rappé, A.K., Casewit, C.L.: Molecular Mechanics Across Chemistry, University Science Books, Sausalito, CA, 1997

65. Rochefort, A. et al.: Electrical and mechanical properties of distorted carbon nanotubes. Phys. Rev. B, 60, 13824-13830, 1999

66. Ru, C.Q.: Axially compressed buckling of a doublewalled carbon nanotube embedded in an elastic medium. J. Mech. Phys. Solids, 49, 1265-1279, 2001

67. Schmidt, B.: On the derivation of linear elasticity from atomistic models. Netw. Heterog. Media, 4, 789-812, 2009

68. Stefanelli, U.: Stable carbon configurations. Boll. Unione Mat. Ital (9), 10, 335-354, 2017

69. Stillinger, F.H., Weber, T.A.: Computer simulation of local order in condensed phases of silicon. Phys. Rev. B, 8, 5262-5271, 1985

70. Tersoff, J.: New empirical approach for the structure and energy of covalent systems. Phys. Rev. B, 37, 6991-7000, 1988

71. Treacy, M.M.J., Ebbesen, T.W., Gibson, J.M.: Exceptionally high Young's modulus observed for individual carbon nanotubes. Nature, 381, 678-680, 1996

72. TuUkKanEN, S. et al.: Stretching of solution processed carbon nanotube and graphene nanocomposite films on rubber substrates. Synth. Met., 191, 28-35, 2014

73. WANG, X., WANG, X., XIAO, J.: A non-linear analysis of the bending modulus of carbon nanotubes with rippling deformations. Compos. Struct. 69, 315-321, 2005

74. Warner, J.H., Young, N.P., Kirkland, A.I., Briggs, G.A.D.: Resolving strain in carbon nanotubes at the atomic level. Nat. Mater., 10, 958-962, 2011

75. Weiner, P.K., Kollman, P.A.: AMBER: Assisted model building with energy refinement. A general program for modeling molecules and their interactions. J. Comput. Chem. 2, 287-303, 1981

76. Yakobson, B.I., Brabec, C.J., Bernholc, J.: Nanomechanics of carbon tubes: instabilities beyond linear response. Phys. Rev. Lett. 76, 2511-2514, 1996 
77. Yu, M.-F., Files, B.S., Arepalli, S., Ruoff, R.S.: Tensile loading of ropes of single wall carbon nanotubes and their mechanical properties. Phys. Rev. Lett. 84, 5552-5555, 2000

78. Zanzotto, G.: On the material symmetry group of elastic crystals and the Born rule. Arch. Ration. Mech. Anal. 121, 1-36, 1992

79. Zhang, D.-B., Dumitriča, T.: Elasticity of ideal single-walled carbon nanotubes via symmetry-adapted tight-binding objective modeling. Appl. Phys. Lett. 93, 031919, 2008

80. Zhao, X., Liu, Y., Inoue, S., Jones, R.O., Ando, Y.: Smallest carbon nanotube is $3 \AA$ in diameter. Phys. Rev. Lett. $92(12), 125502,2004$

MANUEl FRIEDRICH

Applied Mathematics Münster,

University of Münster

Einsteinstrasse 62,

48149 Münster,

Germany.

e-mail: manuel.friedrich@uni-muenster.de

URL: https://www.uni-muenster.de/AMM/Friedrich/index.shtml

and

EdoARdo Mainini

Dipartimento di Ingegneria Meccanica, Energetica, Gestionale e dei Trasporti (DIME), Università degli Studi di Genova,

Via all'Opera Pia 15,

16145 Genova,

Italy.

e-mail: mainini@dime.unige.it

and

Paolo Piovano \& Ulisse Stefanelli

Faculty of Mathematics,

University of Vienna,

Oskar-Morgenstern-Platz 1,

1090 Vienna,

Austria.

e-mail: paolo.piovano@univie.ac.at

URL: https://www.mat.univie.ac.at/ piovano/Paolo_Piovano.html

and

Ulisse STEFANELli

Istituto di Matematica Applicata e Tecnologie Informatiche "E. Magenes" - CNR,

v. Ferrata 1, 27100 Pavia,

Italy.

e-mail: ulisse.stefanelli@univie.ac.at

URL: http://www.mat.univie.ac.at/ stefanelli

(Received June 5, 2017 / Accepted July 11, 2018)

Published online July 18, 2018

(c) The Author(s) (2018) 